\title{
Single molecule fluorescence spectroscopy of the structure and dynamics of the spliceosome
}

\author{
Dissertation \\ for the award of the degree \\ "Doctor rerum naturalium" \\ of the Georg-August-Universität Göttingen
}

within the doctoral program GGNB

of the Georg-August University School of Science (GAUSS)

\author{
submitted by \\ Mira Prior \\ from Steinheim, Westfalen
}

Göttingen 2013 

Members of the Thesis Committee:

\section{Prof. Dr. J. Enderlein (Reviewer)}

3rd Institute of Physics - Biophysics, Faculty of Physics,

Georg-August-University Göttingen

\section{Prof. Dr. R. Lührmann (Reviewer)}

Department of Cellular Biochemistry

Max Planck Institute for Biophysical Chemistry

\section{Prof. Dr. K. Tittmann}

Department of Bioanalytics, Faculty of Biology and Psychology Georg-August-University Göttingen

Date of oral examination: 31.10.2013 


\section{Affidavit}

Hereby, I declare that the presented thesis has been written independently and with no other soureces and aids than quoted.

Göttingen, 19.09.2013 


\section{Contents}

\begin{tabular}{ll}
\hline Abstract & 1
\end{tabular}

1 Introduction 3

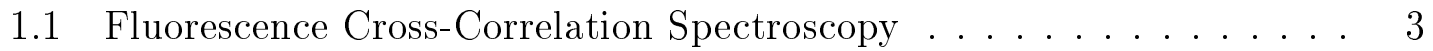

$1.1 .1 \quad$ Applications of FCCS . . . . . . . . . . . . . . . 5 5

1.1.2 Alternative methods for monitoring protein interactions . . . . 7

1.2 The spliceosome $\ldots \ldots \ldots \ldots \ldots \ldots$

1.2 .1 From genes to proteins . . . . . . . . . . . . . 10

1.2 .2 pre-mRNA splicing . . . . . . . . . . . . . . . 11

1.2 .3 RNA processing by the spliceosome - the splice cycle . . . . . 15

1.2 .4 The second catalytic step in detail . . . . . . . . . . . . 18

2 Materials and Methods 23

2.1 Dual-Color Fluorescence Cross-Correlation Spectroscopy . . . . . . . . 23

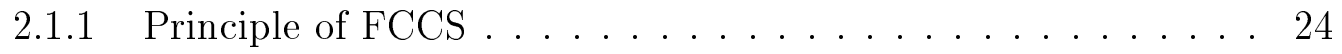

2.1 .2 Theoretical background of FCCS . . . . . . . . . . . 27

2.1 .3 Limitations of FCCS . . . . . . . . . . . . . . 33

2.1 .4 Dual-Focus Fluorescence Cross-Correlation Spectroscopy . . . 35

2.1 .5 Dual-color dual-focus Fluorescence Cross-Correlation Spectroscopy . . . . . . . . . . . . . . . 38

2.1 .6 Used optical setup for the FCCS experiments . . . . . . . . . 39

2.1.7 Computation and evaluation of the correlation data . . . . . 40

$2.2 \quad$ Spliceosome purification, reconstitution, and labeling . . . . . . . . . 43

2.2 .1 The model system . . . . . . . . . . . . . . . . 43

2.2 .2 Spliceosome purification . . . . . . . . . . . . . . 43

2.2 .3 Splicing reconstitution assays . . . . . . . . . . . . . 45

2.2 .4 Denaturing polyacrylamide gel electrophoresis . . . . . . . . 46

2.2 .5 Labeling of spliceosomes . . . . . . . . . . . . . . 46

2.3 Measurement protocol . . . . . . . . . . . . . . . . . . . 48

\begin{tabular}{lll}
\hline 3 & Results & 49
\end{tabular}

$3.1 \quad$ Preliminary experiments for testing the availability of FCCS on the spliceosomal complex . . . . . . . . . . . . . . . 49

3.2 Recruitment and release of Cwc25-Alexa488 studied by FCCS . . . . 55 3.2.1 Binding of Cwc25-Alexa488 to the actin wildtype pre-mRNA. 55 
3.2 .2 Binding affinity of Cwc25 to the $\mathrm{B}^{*}$ complex . . . . . . . . 56

3.2 .3 Binding of Cwc25-Alexa488 to the ActACAC pre-mRNA . . . 58

3.2 .4 Binding of Cwc25-Alexa488 to the Act7wt pre-mRNA . . . . 60

3.2 .5 Binding of Cwc25-Alexa488 to the Act7ACAC mutated premRNA . . . . . . . . . . . . . . 63

$3.3 \quad$ Functionality testing of Slu7-eGFP and Prp16-eGFP . . . . . . . . 65

$3.4 \quad$ FCCS measurements for investigating the binding of Slu7-eGFP . . . 66

$3.5 \quad$ FCCS measurements for investigating the binding of Prp16-eGFP . . 69

3.6 Discrimination of Prp16's and Slu7's binding site on the B* spliceosome 71

\begin{tabular}{lll}
\hline & Discussion & 73
\end{tabular}

4.1 FCCS studies on the spliceosome $\ldots \ldots \ldots \ldots$. . . . . . . . . 73

4.1 .1 Information about binding affinities given by FCCS. . . . . . . 74

4.2 Information obtained by FCCS about the recruitment and release of spliceosomal factors . . . . . . . . . . . . . 76

4.2 .1 Binding behavior of the step 1 factor Cwc25 . . . . . . . . 76

$4.2 .2 \quad$ Binding behavior of Slu7/Prp18 and Prp16 . . . . . . . . . 80

4.2.3 Model for the binding and activity of the step 1 and step 2 splicing factors . . . . . . . . . . . . . . 82

5 Conclusion and outlook $\quad 85$

\begin{tabular}{lr}
\hline Appendix & 87
\end{tabular}

Bibliography . . . . . . . . . . . . . . . . . . . 87

List of Figures . . . . . . . . . . . . . . . . . . . . . . . . 97

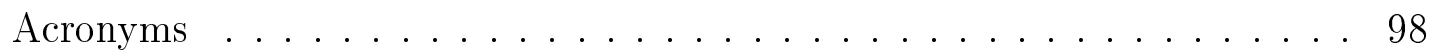

Acknowledgments . . . . . . . . . . . . . . . . . . 101

Curriculum vitae . . . . . . . . . . . . . . . . . . . 103 


\section{Abstract}

Binding reactions can be ideally investigated by Dual-Color Fluorescence CrossCorrelation Spectroscopy (FCCS). Here, this method is used to study the recruitment and dissociation of proteins to and from the spliceosome. The spliceosome is the cellular machinery responsible for removing non-coding introns from precursor mRNA (pre-mRNA).

The spliceosome assembles on a pre-mRNA, which consists of two exons and one intron, stepwise by the binding of five snRNPs and several other proteins. This leads to the formation of the B complex which has no active catalytic center yet. Structural rearrangements lead to the activated $\mathrm{B}^{\text {act }}$ complex which is formed by further rearrangements in the catalytically active $\mathrm{B}^{*}$ complex. The $\mathrm{B}^{*}$ complex catalyzes the first splicing step in which the branch site (BS) attacks the 5' splice site (5'SS). The 5' exon is cleaved and the intron-3'-exon lariat is formed. The resulting $\mathrm{C}$ complex then catalyzes the second splicing step. The intron is cleaved and both exons are joined together forming mature mRNA. During both catalytic steps, the spliceosome undergoes many changes in its protein composition, and several structural rearrangements occur.

These changes and rearrangements can be investigated by standard biochemical methods. However, these methods often do not provide data about dynamics, which are necessary for understanding the recruitment and release of particular molecules. One method which provides these information is FCCS. In contrast to methods like mass spectrometry, in FCCS measurements it is possible to observe reactions in real-time and at equilibrium without further biochemical perturbation of the sample. FCCS works at low nanomolar concentrations and only small sample volumes are necessary. Using FCCS, it could be determined how changes in the spliceosome composition and conformation occur (simultaneously or consecutively). The roles of certain spliceosomal RNA helicases in the restructuring of the complex could be investigated. FCCS enables the observation of protein-protein interactions and the determination of binding constants for proteins to the spliceosome.

In order to better understand the dynamic nature of the spliceosome during its catalytic activation, the step 1 factor Cwc25 and the step 2 factors Slu7, Prp18, and Prp16 were investigated. Their role in the maturation process, the fundamental question of the time point and manner of their recruitment has not been answered yet. 
With FCCS, the binding of the step 1 factor Cwc25 was observed. It was shown that Cwc25 has a high-binding affinity to the spliceosome after the Prp2-mediated rearrangements into the catalytically activated $\mathrm{B}^{*}$ complex. The high-binding affinity was reflected in a strong binding constant of $30 \mathrm{pM}$ which was measured with FCCS.

By using several mutant pre-mRNAs it could be demonstrated that Cwc25's release does not depend on the second catalytic step per se. Its dissociation depends on the docking of the 3'SS to the active site and the action of Prp16 and Slu7/Prp18. It was also observed that the distance between the branch site and 3'SS influences the release of Cwc25 and the second catalytic step. If the distance between BS and 3'SS is short (e.g. 7 nucleotides long), the distance between the 3'SS and the active site is also small. The 3'SS can dock into the active site without the further stabilization by Slu7/Prp18. The activity of Prp16 and the docking event lead to the release of Cwc25. Slu7/Prp18 can stabilize the system and thereby induce further release of Cwc25. If the distance between BS and 3'SS is longer (e.g. 38 nucleotides), Slu7/Prp18 has to stabilize the interaction of the 3'SS and the active site and the step 2 conformation. In this case, Prp16 alone cannot induce the release of Cwc25. Cwc25 does dissociate from the spliceosome after the action of Prp16 and in the presence of Slu7/Prp18.

It was shown further that Prp16 and its ATPase activity are necessary for the formation of a functional step 2 active site. Slu7/Prp18 are required for the efficient docking of the 3' splice site (3'SS) to the active site. The FCCS experiments showed that Prp16 and Slu7/Prp18 have distinct binding sites in the spliceosome which are formed during the catalytic activation. During activation their first low-affinity binding sites are transformed into high-affinity binding sites. Both proteins are bound to the spliceosome at an early stage so that they are present before their catalytic function is actually required in the process.

Here, it was shown that FCCS is ideally suited to investigate macromolecular protein complexes like the spliceosome and is a powerful tool for studying quantitatively spliceosomal protein dynamics at equilibrium. 


\section{Introduction}

Many biochemical methods which are used to study macromolecular complexes, e.g. the spliceosome or ribosome, provide only semi-quantitative results. They yield only little information about dynamics, which are necessary for understanding the binding behavior between proteins and proteins or proteins and complexes. A method that provides data about dynamics in real-time is Dual-Color Fluorescence CrossCorrelation Spectroscopy ( $(\mathrm{FCCS})$. This method is an ideal tool to quantitatively study the protein dynamics of the spliceosome.

\subsection{Fluorescence Cross-Correlation Spectroscopy}

FCCS is an extension of Fluorescence Correlation Spectroscopy (FCS). FCS was introduced in the 1970s by Magde et al. 11213. It allows for the observation of e.g. photophysical dynamics of fluorescent molecules 4 [5 6, conformational dynamics ${ }^{7}$ 8], interactions of macromolecules 9 , and biochemical kinetics [10] 11. FCS requires single molecule sensitivity for these observations. The technique is applicable to freely diffusing, fluorescently labeled molecules in solution. The fluorescence fluctuations of a signal arising from molecules diffusing through the confocal volume are analyzed with statistical methods. By calculating a temporal auto-correlation of the signal, diffusion coefficients and concentrations of the fluorescently labeled molecules can be determined ${ }^{12}$. Information about binding reactions between different molecules are less accessible with FCS. There are different reasons why FCS is not useful for monitoring binding reactions:

First of all, the diffusion coefficient depends only weakly on the molecular weight. According to the well known Stokes-Einstein equation, the diffusion coefficient is inversely proportional to the hydrodynamic radius 13 . The hydrodynamic radius in turn depends on the cubic root of the molecular weight. Small changes in the molecular weight will thus barely influence the molecule's diffusion coefficient. Binding reactions, which are only accompanied by such small changes in molecular weight, can therefore hardly be observed in FCS measurements 14. Additionally, aggregates can distort the correlation curves, which further complicates the analysis.

Another problem in applying FCS in binding studies is the confocal detection volume. The size and shape of the detection volume depend on the laser intensity and are sensitive against refractive index mismatch between the sample and the objective's immersion medium. The diffusion coefficient in turn depends on the shape 
and size of the detection volume. The resulting distortions introduce errors in an FCS experiment for binding studies 14 .

Additionally, FCS is severely affected by variations in the molecular brightness (counts per second per molecule) of a fluorophore. The molecular brightness is strongly influenced if two fluorescently labeled molecules are bound to each other. Statistical labeling of multiple sites in one molecule also leads to a broad distribution in the molecular brightness 14 . Both of these contributions introduce errors during FCS evaluation.

These limitations can be overcome by FCCS, which is based on FCS and retains the high sensitivity of that method. The main difference between both methods is the use of two excitation lasers at two different wavelengths in FCCS instead of one laser at one wavelength in FCS. In FCCS, the spontaneous fluorescence fluctuations of two molecules labeled with different fluorescent dyes are detected separately and compared to each other. This is done by a cross-correlation analysis ${ }^{[15}$, which provides information about the binding behavior of the two fluorescently labeled species. If the molecules are not bound, the fluorescence signals are uncorrelated. If the molecules are bound to each other, there is a correlation between the fluorescence signals. This correlation yields a cross-correlation curve with an amplitude which is higher than that of an FCCS measurement on a sample with unbound molecules ${ }^{16}$. The amplitude is directly proportional to the fraction of bound molecules.
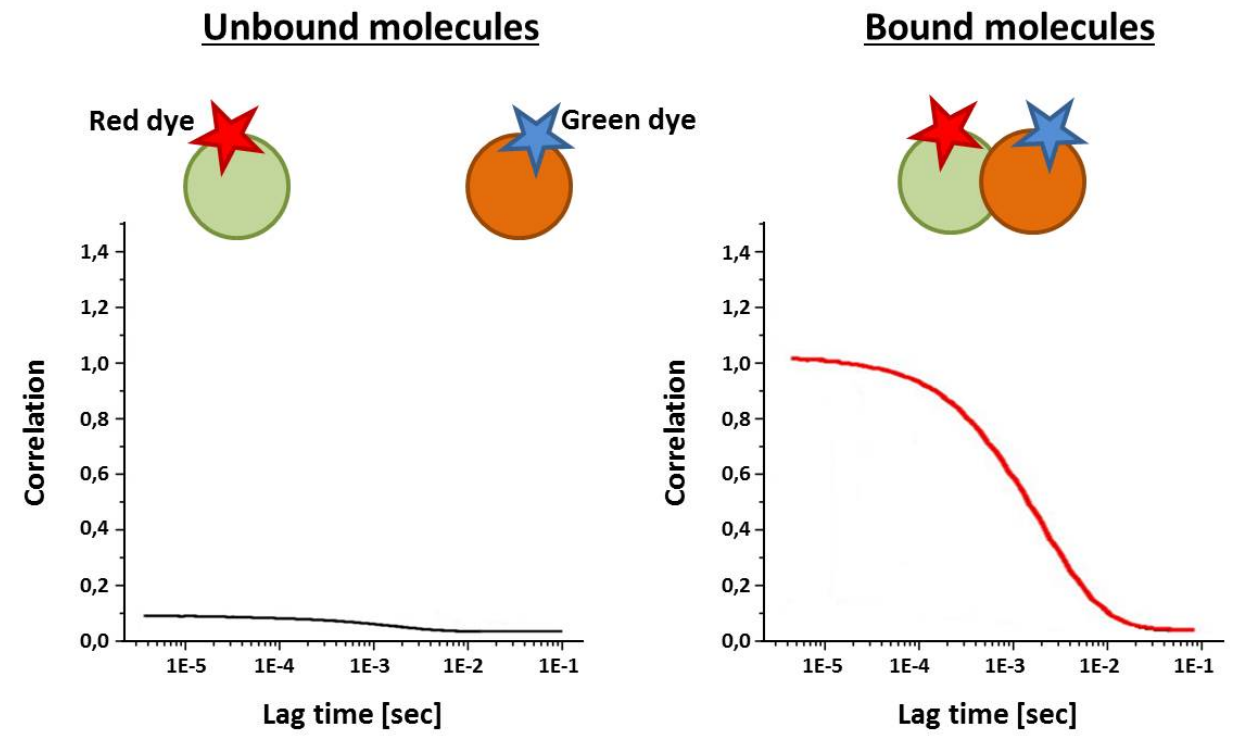

Figure 1: If the two fluorescently labeled molecules are not bound to each other, the crosscorrelation amplitude is small. If the molecules are bound, the cross-correlation amplitude is large. 
In contrast to many biochemical and physical methods, FCCS has several advantages, e.g it yields quantitative information about dynamics of recruitments and dissociations. In particular, FCCS is a useful method for determining protein and RNA interactions because it works at nanomolar concentrations and low sample volumes are necessary. Additionally, it can be applied at equilibrium without further biochemical perturbation of the sample and investigation of reactions in real-time are possible 12$][10$.

\subsubsection{Applications of FCCS}

The first theoretical concept of FCCS was developed by Eigen et al. ${ }^{18}$. The first experiments with FCCS were done by Schwille et al. 19 , who used the cross-correlation between two fluorescent signals to observe the hybridization kinetics of two oligonucleotides labeled with different fluorophores. For the experimental realization, they used a confocal setup with two lasers. The laser beams were focused on the same spot. By using two spectrally resolved detectors, the fluorescence signals could be detected in a wavelength-sensitive way 19 .

In the next approach, Rigler et al. used FCCS to detect amplified DNA sequences in a polymerase chain reaction. They monitored the formation of double-stranded DNA with two fluorescently labeled primers 20]. The doubly labeled DNA strands were further used in the study of Kettling et al. 10, where a DNA substrate was fluorescently labeled with two dyes at opposite ends and the enzymatic cleavage of the DNA by an endonuclease was monitored. They could quantify the enzymatic activity by measuring reaction rates.

In addition to DNA-DNA interactions, protein-protein interactions have been observed. Bieschke et al. used FCCS to monitor prion-protein aggregations 21. In the study of Varghese et al., FCCS was used to investigate the diffusion behavior, binding kinetics, and effect of small organic molecules on the binding of IgG and Fab fragments of anti-IgG molecules 22 . This work demonstrated the usefulness of FCCS for kinetic studies on freely diffusing molecules in solution.

Further quantitative investigation of ligand binding was developed by Weidemann et al. They took into account the focal geometry, background signal, and cross-talk as well as photophysical and biochemical effects ${ }^{23}$. These parameters can influence the brightness of the fluorophores and thereby the fluorescence signals which were used for analysis. A practical guideline for FCCS measurements which considered the limitations and corrections of these experiments was published by Bacia et al. 24 . 
Several further studies dealt with the limitations and theoretical foundations of FCCS. FCCSs main intrinsic problem is cross-talk. Cross-talk results from crossexcitation and cross-emission. In the case of cross-excitation, the dye with the higher wavelength is excited by the laser with the shorter wavelength. Cross-emission results from the coupling of the fluorescence signal of the dye with the shorter excitation wavelength into the detection channel for the longer wavelength. Corrections for cross-talk and quenching have been given in the work of Földes-Papp ${ }^{15}$. He also provided a theoretical overview about FCCS. Crosstalk in FCCS can be experimentally avoided by using appropriate excitation and emission filters.

Instead of exciting the sample with a continuous wave laser like in the work of Schwille 19], two alternatingly pulsed lasers can be used 25. This results in suppressing cross-talk by using the fluorescence lifetimes of the fluorophores. The fluorescence lifetime is the average time of a molecule in the excited state before emitting a photon and returning into the ground state. The conventional way to measure fluorescence lifetimes is Time-Correlated Single-Photon Counting (TCSPC). In a TCSPC experiment, the sample is excited with a pulsed laser. Single fluorescence photons are then detected and their arrival times in relation to the excitation pulse are measured. Finally, the photons are sorted according to their arrival times in a histogram. This histogram reflects the exponential decay of the fluorescence intensity and allows for the determination of the fluorescence lifetime. By measuring the fluorescence lifetimes of two different fluorophores, it is possible to distinguish between the fluorescence photons of both dyes and reduce cross-talk. Thews et al. used this method to investigate the binding behavior of proteins in live cells 25. Beside this work, a lot of FCCS studies were performed on cells 26 27 28. By applying FCCS on and in cells, studies on many different parts of living cells are possible. Weidemann et al. for example investigated the ligand-receptor interactions in the plasma membrane 14 .

A limitation in FCCS measurements is that the two detection volumes have to overlap to excite the two different fluorophores simultaneously. To achieve this experimental, the two lasers have to be aligned to the same spot. The difficulty of alignment can be avoided by using Single-Wavelength FCCS (SW-FCCS). In this method, two different fluorophores are used which are excitable with the same wavelength but have different emission wavelengths. Therefore, the difference between the maximum in the absorption spectra and the maximum in the emission spectra, the so-called Stokes shift, differs for both fluorophores. The fluorescence signals are detected in two spectrally distinct detection channels [29]30. The advantage of 
SW-FCCS is the easier alignment of only one laser beam instead of two. The disadvantage of this method is higher cross-talk, which cannot be suppressed completely. Another approach for avoiding non-ideally overlapping detection volumes is the combination of FCCS with two-photon excitation. In the work of Heinze et al., a twophoton laser was used to excite two fluorophores with the same excitation wavelength but different emission wavelengths 3132 . During the excitation, two photons are simultaneously absorbed by a fluorophore, exciting the molecule into a higher electronic state. The sum of the energy of both photons has to correspond to the energy which is necessary to excite the molecule 16. The excitation spectra of a fluorophore of two-photon excitation can differ from the spectra upon one-photon excitation, whereas the emission will be the same. FCCS experiments with twophoton excitation were shown for two different fluorophores, i.e. Rhodamin green and Texas red, which can be excited simultaneously but showed different emission spectra 31. Similar to SW-FCCS, the exact alignment of two laser beams is not necessary. Finding suitable fluorescent dyes, however, can be a problem.

In this work FCCS was used to investigate recruitment and dissociation orders of proteins in the yeast spliceosome. The suitability of FCCS for measuring binding constants of a small protein to a large macromolecular complex is tested. Changes in the binding strength of proteins in a complex system are observed and a further application is the determination of protein composition.

\subsubsection{Alternative methods for monitoring protein interactions}

Another and widely used method to determine the protein composition of a biological sample is Mass Spectrometry (MS). With this method, a multiprotein complex, e.g. the nuclear pore complex or the spliceosome, can be investigated. For a mass spectrometry measurement, the biological sample first has to be ionized and transferred into the gas phase. One method for the ionization step is Matrix-Assisted Laser Desorption Ionization (MALDI). This is a non-destructive ionization and vaporization method in which biomolecules are imbedded in a solid matrix. By irradiating the matrix with a UV laser pulse in the presence of a high-voltage electric field, the biomolecules are ionized and accelerated. After their acceleration, the mass-tocharge ratio of the ionized biomolecules is analyzed with e.g. a time-of-flight (TOF) analyzer. TOF analysis is based on comparing the kinetic energies of the respective molecules. After acceleration, all molecules have the same kinetic energy. The kinetic energy depends on the mass and velocity of the molecule, i.e. molecules of 
the same kinetic energy but different molecular weight will have different velocities. The TOF analyzer measures the differences in velocities of the ionized biomolecules. In most cases, electron multipliers are used for detection 16. In the end, the results of the mass spectrometry measurements are compared to values from a protein database. Thereby, the components of a complex biological sample can be identified. In many biological complexes, e.g. the spliceosome, the ordered recruitment and subsequent release of certain proteins is important. Mass spectrometry is a sensitive method, which gives good indications about the relative amount of a protein in a complex system, i.e. the spliceosome. However, only semi-quantitative information about dynamics induced by certain proteins and their binding and release are provided. To gain more information, complex further purification steps are necessary. These can be avoided by using FCCS which enables measurements in real-time and at equilibrium without further biochemical purification.

Another method for monitoring protein-protein interactions is Fluorescence Anisotropy (FA). This method is based on the polarization of a fluorescence signal. During an FA measurement, a fluorophore attached to a molecule is excited using linearly polarized light. The intensity of the fluorescence emission is then measured through a polarizer. By changing the orientation of the polarizer, the intensities with polarizations parallel and perpendicular to the excitation polarization can be measured. The anisotropy describes the degree of polarization of a sample.

Most fluorophores absorb light in a preferred direction, namely parallel to the absorption transition moment of the fluorophore. For absorption, the electric dipole of a fluorophore does not need to be aligned exactly with the axis, among which the excitation light is polarized. The probability of absorption is proportional to $\cos ^{2}(\Theta)$ with the angle $\Theta$ between the absorption dipole and the axis. Due to Brownian motion a molecule starts to rotate. Observing the emission intensities with polarization parallel and perpendicular to the excitation polarization over time allows for measuring changes in the intensities due to the rotational movement of the molecule. The excited molecule will be randomized and the anisotropy will decay. The dependance of the anisotropy on the time is measured by the time-resolved anisotropy. This is an exponential decay with a characteristic time constant, i.e. the rotational correlation time. This factor depends on the viscosity and temperature of the surrounding medium, and the volume of the rotating molecule. It is also related to the rotational diffusion coefficient. Binding interactions between different molecules can change the rotational diffusion coefficient of the molecules and thereby the anisotropy. 
Fluorescence Anisotropy has several limitations. First, the fluorescence lifetime of the fluorophore has to be similar to the rotational correlation time. If the lifetime is shorter than the rotational correlation time, the fluorescence light is emitted before the molecule rotates completely. In this case, FA is not suitable for observing binding reactions between molecules. Second, the local motion of the fluorophore which is attached via a linker to the molecule of interest can influence the anisotropy. Segmental motion of the linker between the fluorophore and the molecule without motion of the macromolecule itself contributes to a depolarization of the sample and can influence the observation of binding reactions. Application of Fluorescence Anisotropy on a macromolecular complex can be challenging.

In this work, which was a cooperation with the group of Prof. Lührmann from the MPI for biophysical chemistry, FCCS was used to study the spliceosome. While the biological part (sample preparation and biochemical characterization of the samples) was done in the Lührmann lab, the spectroscopy part including the entire data analysis and evaluation was done by myself. 


\subsection{The spliceosome}

\subsubsection{From genes to proteins}

The whole genetic information of higher organisms is encoded in their genome in form of DNA. The DNA sequence contains the information for the assembly and function of proteins. The information transfer from genes to proteins proceeds over different steps and with the help of a lot of macromolecular complexes, e.g. the spliceosome and ribosome (see fig. 2).

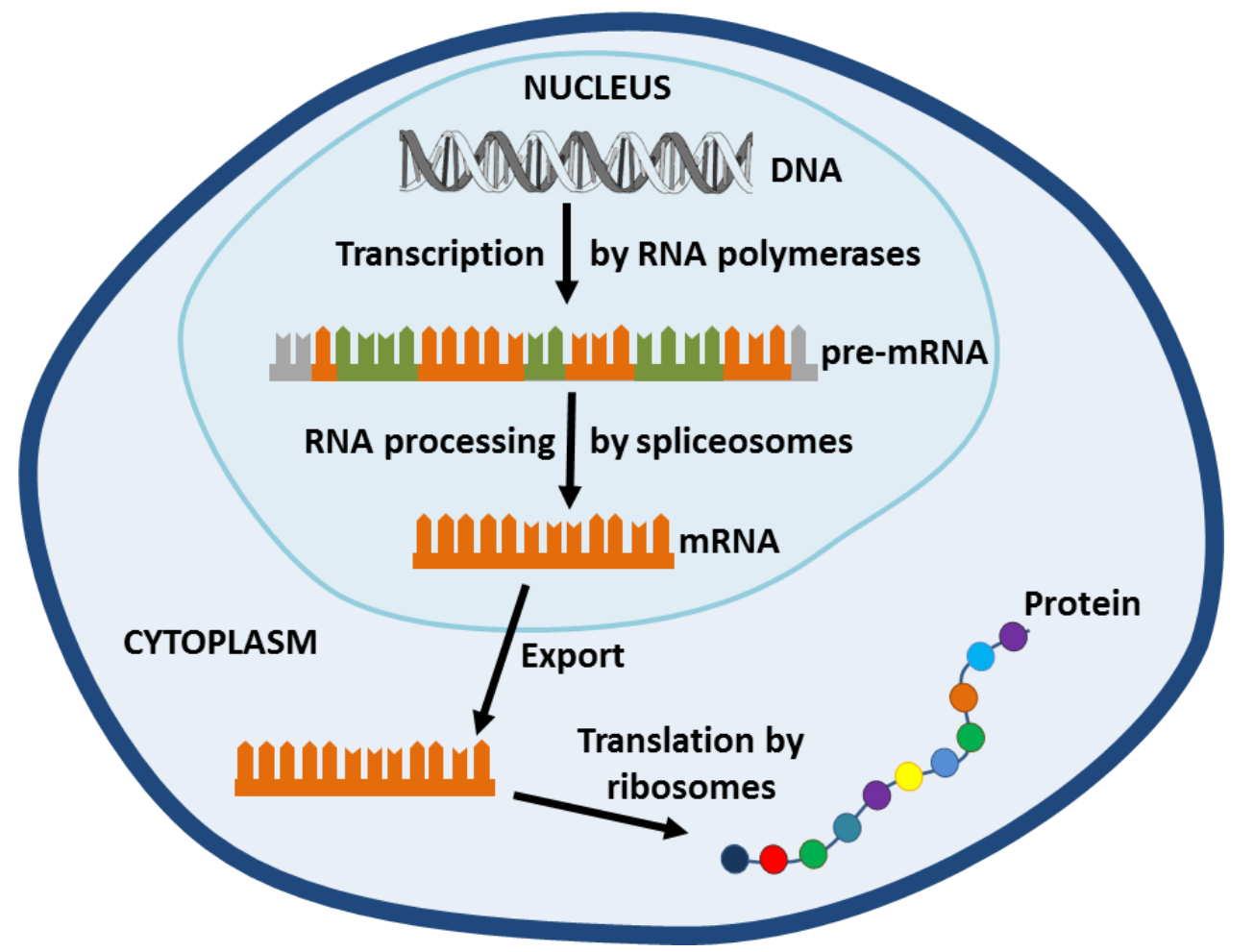

Figure 2: An overview of information flow from genes to proteins. The DNA is transcribed into pre-mRNA. The pre-mRNA is processed into mRNA by the spliceosome. The mRNA is transported out of the nucleus into the cytoplasm via a nuclear pore complex.

In the cytoplasm the mRNA is translated into polypeptides by ribosomes.

First, the DNA is transcribed into precursor-messenger RNA (pre-mRNA). During this process, one of two DNA strands is complementary copied into pre-mRNA and the transcribed pre-mRNA contains the same information. Responsible for the transcription is a complex enzyme, i.e. the RNA polymerase. In eukaryotes three RNA polymerases exist: RNA polymerase I, II, and III, which transcribe different RNAs. 
Next, the pre-mRNA has to be processed. The RNA processing includes different steps. Two processes which occur during and immediately after transcription are the modification of the 5' end and 3' end. At the 5' end a 5' cap is added. This is a modified guanine nucleotide. To the 3' end, a poly(A) tail is linked. Both modifications, capping of the 5' end and polyadenylation of the 3' end, are important for the nuclear export, translation, and stability of mRNA. A third process has to be performed because of the mosaic structure of eukaryotic genes which contains exons and introns. The exons are segments of the sequence which are coding for the synthesis of proteins. The introns are the non-coding segments. Genes with exons and introns are widespread among eukaryotes. The introns of simpler eukaryotes (e.g. yeast) are smaller in number and in size than those of plants or animals. Because the introns do not contribute to the later protein synthesis, they have to be removed. This is done by a process called splicing. During splicing, the pre-mRNA strand is cut at both ends of the intron and the exons are covalently joined. Thereby, the pre-mRNA is converted into mRNA, which contains only the exons 33134 .

Transcription and RNA processing proceed in the cell nucleus, whereas protein synthesis occur in the cytoplasm. Thus, in the next step the mRNA has to be transported out of the cell nucleus through the nuclear pore complex into the cytoplasm. There, the translation of the mRNA into a polypeptide chain by the ribosome occurs. Ribosomes are complex ribonucleoprotein machines consisting of ribosomal RNA and ribosomal proteins $33[34$.

Overall, splicing is an important step in gene expression because precise splicing of pre-mRNA is essential for synthesis of functional proteins 34 .

\subsection{2 pre-mRNA splicing}

The spliceosome cleaves the introns in a very precise reaction out of the pre-mRNA. This is possible by conserved sequences within the exon and intron. Studies on many eukaryotic systems showed three conserved consensus sequences: the 5' Splice Site (5'SS), the 3' Splice Site (3'SS), and the Branch Site (BS). The 5'SS is located at the 5' end of an intron and the 3'SS at the 3' end of an intron. The BS is located between 10 to 155 nucleotides upstream of the 3'SS. All three elements are highly conserved in S.cerevisiae $\stackrel{35[36}{ }$ and the conserved sequences at the exon-intron border of yeast and humans are shown in figure 3 . 
Saccharomyces. cerevisiae:

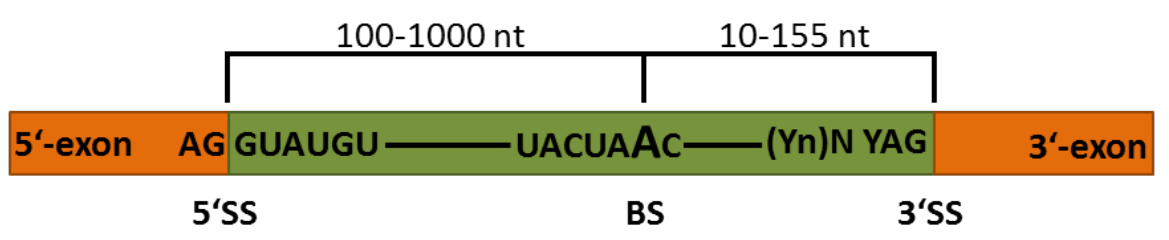

Homo sapiens:

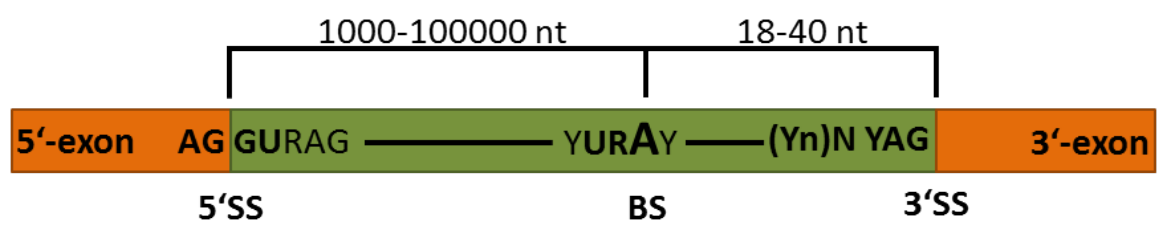

Figure 3: Schematic representation of conserved sequences in introns of S. cerevisiae and homo sapiens. The branch site (BS) adenosine is highlighted. Bold letters indicate a $90 \%$ or higher conservation of the respective nucleotide. $Y$ stands for pyrimidines, $R$ for purines, $N$ for a $G$ or $G X$ and $X$ for $A$ or $G$ or $U$ or $C$. (Yn) denotes the polypyrimidine tract and the numbers indicate the distance between the 5'SS and the $B S$ and between the BS and the 3'SS. (Figure also appeared in the PhD thesis of Peter Odenwälder)

In nature, two different types of splicing occur: self-splicing and splicing by the spliceosome. In the process of self-splicing, RNAs can remove their introns without the help of proteins. For non self-splicing introns, the spliceosome, which is a ribonucleoprotein complex, has to process the pre-mRNA splicing. $\frac{37}{37}$

The self-splicing introns are sorted into two groups. The group I introns are found commonly in fungal and plant mitochondria, plant chloroplasts, and in nuclear RNA of lower eukaryotes (e.g. Tetrahymena). The nucleotide sequences of group I introns are variable, but all of them form a similar three-dimensional structure. Together with several conserved nucleotides, the structure allows the group I introns for catalyzing the removal of the intron by themselves. For group I introns, the splicing reaction is started by binding of a $G$ nucleotide to the intron sequence. This $G$ nucleotide is activated to form an attacking group which will break the 5'SS $34 \mid 38$. Group II self-splicing introns are found in the pre-mRNA of fungal and yeast mitochondria, and plant chloroplasts. In the self-splicing process of group II introns, the 5' splice site is cleaved first. Then the 5'SS is covalently bound to an adenosine residue near the 3'SS of the intron and a structure called lariat is formed. Here, the A nucleotide in the intron sequence is the attacking group. In the last step, the 
3'SS is cut, the intron is released, and the ends of the exons are bound covalently together 3438 .

There are similarities between the group I and group II introns. For both groups the sequence of the intron is important, because the intron is folded into a threedimensional structure which brings the ends of the intron in contact $34 \mid 38$.

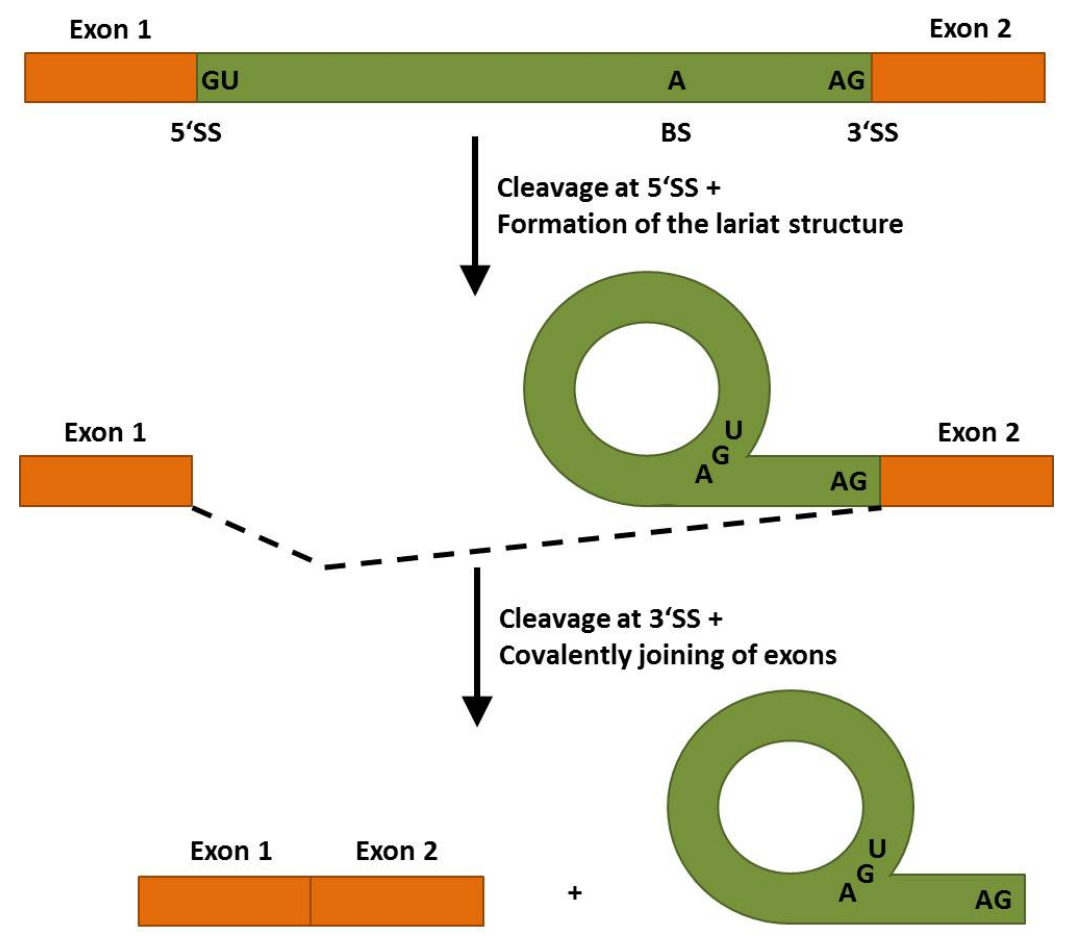

Figure 4: Principle of pre-mRNA splicing. The cleavage of the 5'SS and the formation of the lariat structure during the first step are shown. In the second step, the 3'SS is cleaved and the exons are ligated. (Adapted from Cooper ${ }^{[39]}$.)

In contrast to the self-splicing introns, the introns of pre-mRNAs in animal cells do not splice themselves. The steps are similar to the splicing of group II introns, but a macromolecular complex, the spliceosome, is necessary to process the intron removal. The spliceosome consists of a large number of proteins and five ribonucleoparticles called SnRNP\$ (small nuclear ribonucleoproteins). The snRNPs consist of a small nuclear Ribonucleic Acid (snRNA), seven Sm proteins, and a variable number of specific proteins 40 . Because of their high amount of uracil, the spliceosome snRNPs are denoted as the U1, U2, U4/U6, and U5 snRNP. The RNA molecules of the snRNPs are relatively short with less than 200 nucleotides, but are involved in the major part of pre-mRNA splicing.

The removal of an intron proceeds through two sequential phosphoryl-transfer reactions known as transesterifications 41 . In the first step, the adenosine of the BS 
attacks the 5'SS nucleophilically, generating the free 5' exon and the lariat-3'-exon intermediates. In the second step, the 3' hydroxyl group of the 5' exon attacks the 3'SS. In the end, the exons are ligated and the intron lariat is released from the complex 40 (see fig. 4).

Pre-mRNAs can be spliced in different ways in a process called "Alternative Splicing". This occurs commonly in human cells and results in the generation of different mRNAs from the same pre-mRNA. These lead to different polypeptide chains from one gene. During alternative splicing, particular exons may be included within, or excluded from, the pre-mRNA (see fig. 5).

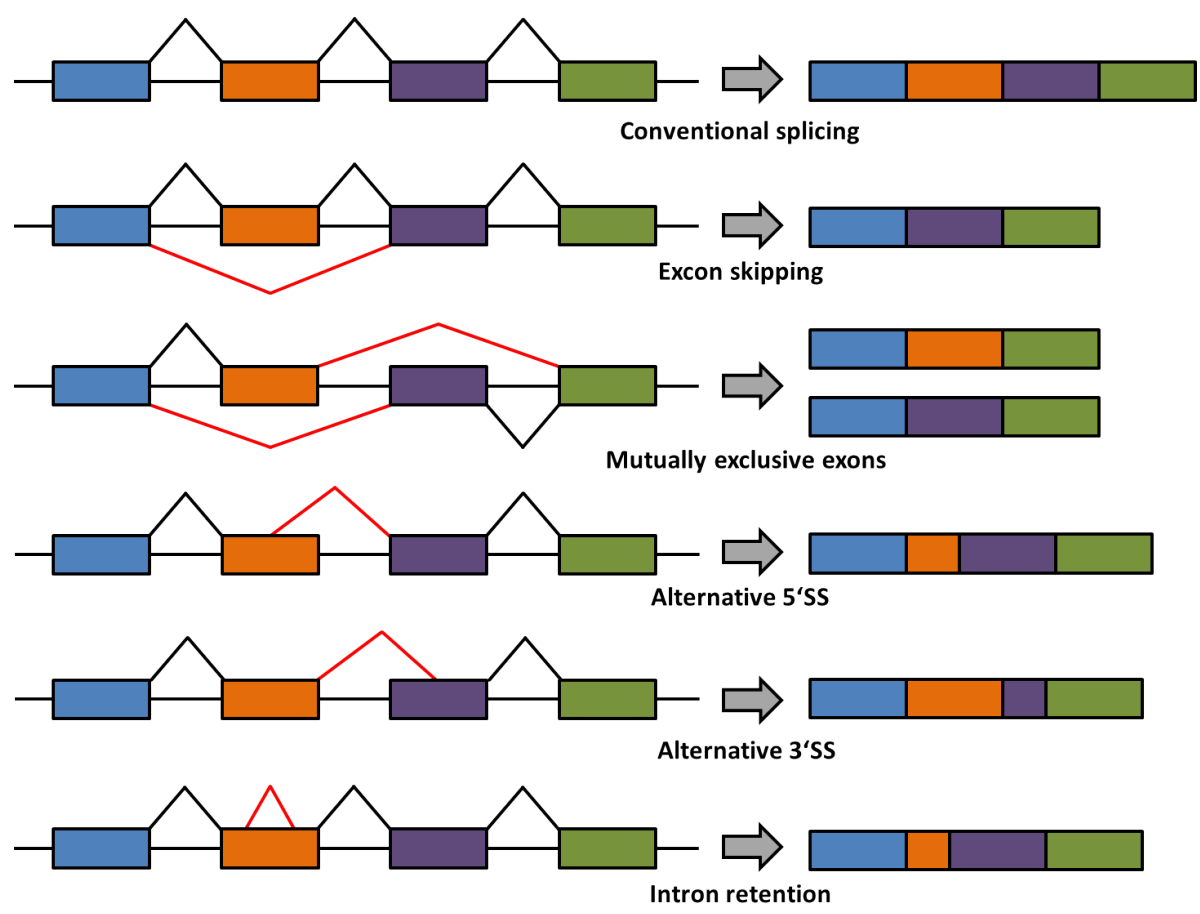

Figure 5: Scheme of the different modes of splicing: conventional splicing, exon skipping, mutually exclusive exon splicing, alternative 5'SS splicing, alternative 3'SS splicing, and intron retention.

In general, five different modes of alternative splicing can be distinguished: In the exon skipping case, an exon is spliced out or kept in the sequence. It is the most common mode in mammalian pre-mRNAs 42 . In the case of mutually exclusive exons, one exon is always kept in the mRNA, while a second one is always spliced out. In the next case, different alternative 3'SS or 5'SS can be used. In the mode of intron retention, a sequence can be spliced out as an intron or is retained 142 . 


\subsubsection{RNA processing by the spliceosome - the splice cycle}

The spliceosome does not exist as a macromolecular complex before it processes the pre-mRNA, but is assembled on the pre-mRNA in different steps. During these steps, the snRNPS and multiple proteins bind to the pre-mRNA. After the assembly of the spliceosome, the snRNPS and several splicing factors catalyze the removal of the intron and the joining of the exons ${ }^{34}$. The process of splicing is done in a stepwise manner and can be described with a splice cycle (see fig 6).

The recognition of the 5'SS, 3'SS, and BS is done by base-pairing between the snRNAs and the consensus sequences in the pre-mRNA. During the complete splicing process, different base-pair interactions are broken and new interactions are formed. The RNA sequences have to be checked multiple times before the chemical reaction of base-pairing. The control mechanism increases the accuracy of splicing 38 .

During pre-mRNA splicing, the spliceosome hydrolyzes many ATP molecules. While, ATP hydrolysis is not required for the chemistry of splicing per se, the assembly of the spliceosome and the rearrangements over the complete splicing process require ATP hydrolysis. Using the energy provided by ATP hydrolysis, the additional proteins of the spliceosome rearrange RNA-RNA interactions. All RNA-RNA rearrangements which need ATP occur between the snRNPs themselves and between snRNPS and the pre-mRNA. The remodeling steps in the yeast spliceosome are driven by at least eight evolutionarily conserved DExH/D-box ATPases or RNA helicases 43 . DExH/D-box helicases are a family of proteins and their function is the unwinding of RNA 44. For this process they use the energy from the hydrolysis of ATP or other nucleotide triphosphates.

The process of splicing occurs via the assembly of the spliceosome, followed by rearrangements of the RNA-RNA network, and thereby, creation of an active catalytic center in the spliceosome. This catalytic center is then formed by RNA molecules. The complicated but particular effective way of forming an active site prevents unpredictable splicing 38 .

In the first step of the splice cycle the U1 snRNP recognizes the 5'SS by basepairing. This is an ATP-independent reaction and leads to formation of the E complex. Then, the U2 snRNP binds to the BS by base-pairing (see fig. 6) and the A complex is formed. In the next step, the pre-formed U4/U6.U5 tri-snRNP is recruited to the spliceosome. The formed spliceosomal complex is denoted as the B complex which does not yet have an active catalytic center. The spliceosome is subsequently acti- 


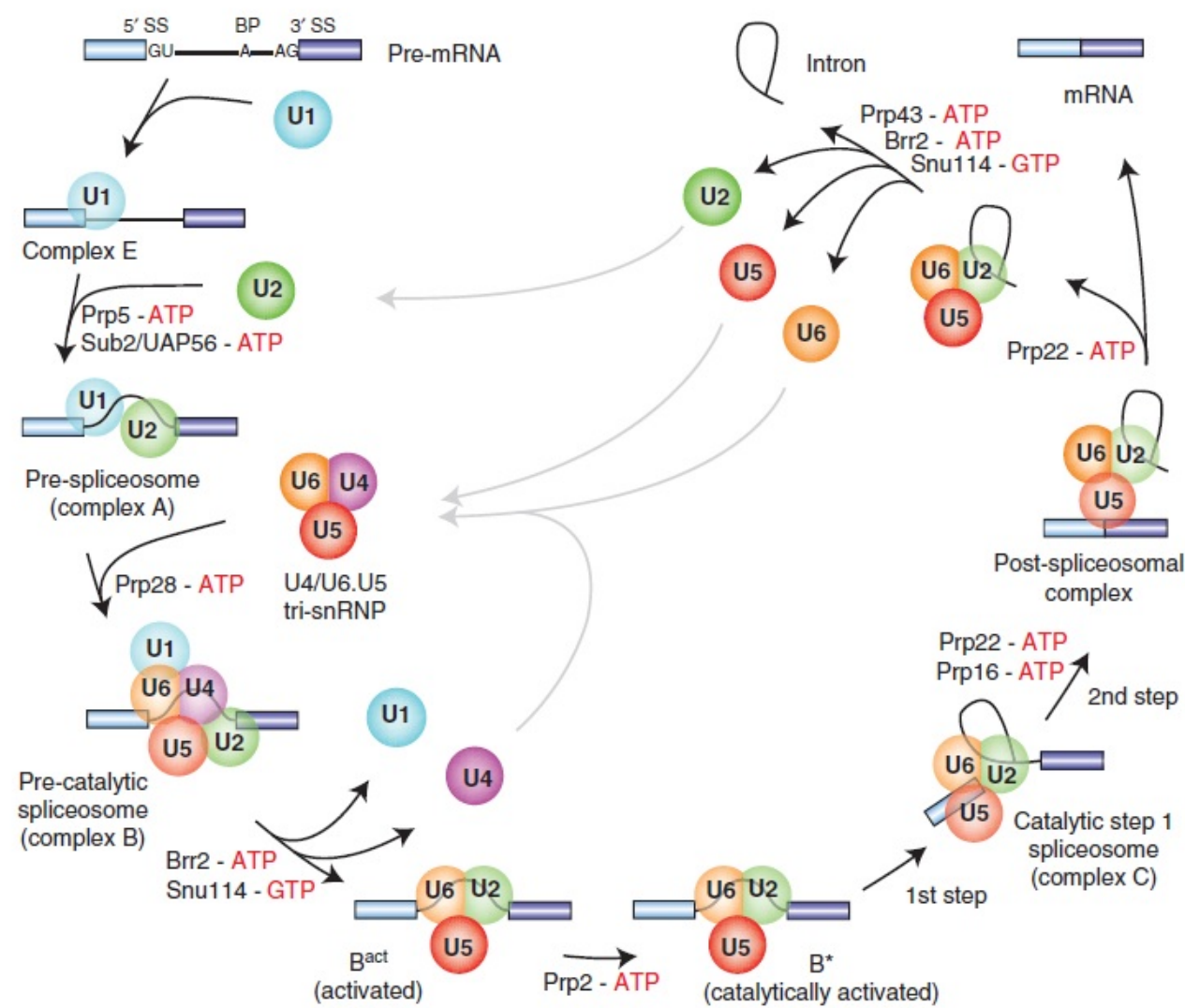

Figure 6: The assembly cycle of the spliceosome. (Figure taken from 40$]$ )

vated for the first catalytic step: first, by the action of the DExH/D-box ATPases Prp28 and Brr2, in cooperation with the GTPase Snu114 and second, by the remodeling of the spliceosome's RNA-RNA interaction network (this complex is termed the $\mathrm{B}^{\text {act }}$ complex). The necessary rearrangements include the displacement of the U1 and U4 snRNAs and the formation of new base-pair interactions of U6 snRNA with U2 snRNA and the 5'SS. The resulting RNA structure forms the core of the catalytic center 45] 446. During the transition into the $\mathrm{B}^{\text {act }}$ complex, all U1 and U4/U6 proteins are released and approximately 20 proteins are stably recruited. There are large changes in the protein composition (see figure 7). Part of the recruited proteins are eight proteins of the Nineteen Complex (NTC) and 12 additional proteins, which are denoted as NTC-related proteins 47 37 [48. The $\mathrm{B}^{\text {act }}$ complex then has a catalytic center which is inactive.

For the first catalytic step of the splice cycle three proteins are necessary: Prp2, Spp2, and Cwc25 499 50 51 52. Prp2 is a DEAH-box ATPase and Spp2 is its coactivator. Both proteins are needed for the ATP-dependent creation of the active site of the catalytic center for the first step and large changes in the structure of 
the spliceosome are induced by their action $50,48,53$. The newly formed complex is called the $\mathrm{B}^{*}$ complex. During the transition from the $\mathrm{B}^{\text {act }}$ into the $\mathrm{B}^{*}$ complex, a substantial structural change occurs. This is seen by a shift in the sedimentation rate of the $\mathrm{B}^{\text {act }}$ complex $(45 \mathrm{~S})$ into the $\mathrm{B}^{*}$ complex (40 S). Additionally, changes in the composition of the spliceosome occur. Two NTC-related proteins, Cwc24 and Cwc27, are displaced and the binding of the U2 SF3a/SF3b and RES complex proteins is destabilized 54 . Because of the rearrangements, the adenosine of the BS becomes accessible for the nucleophilic attack of the 5'SS 48 53. Thereby, the free 5 ' exon and the lariat-3'-exon intermediate are generated.

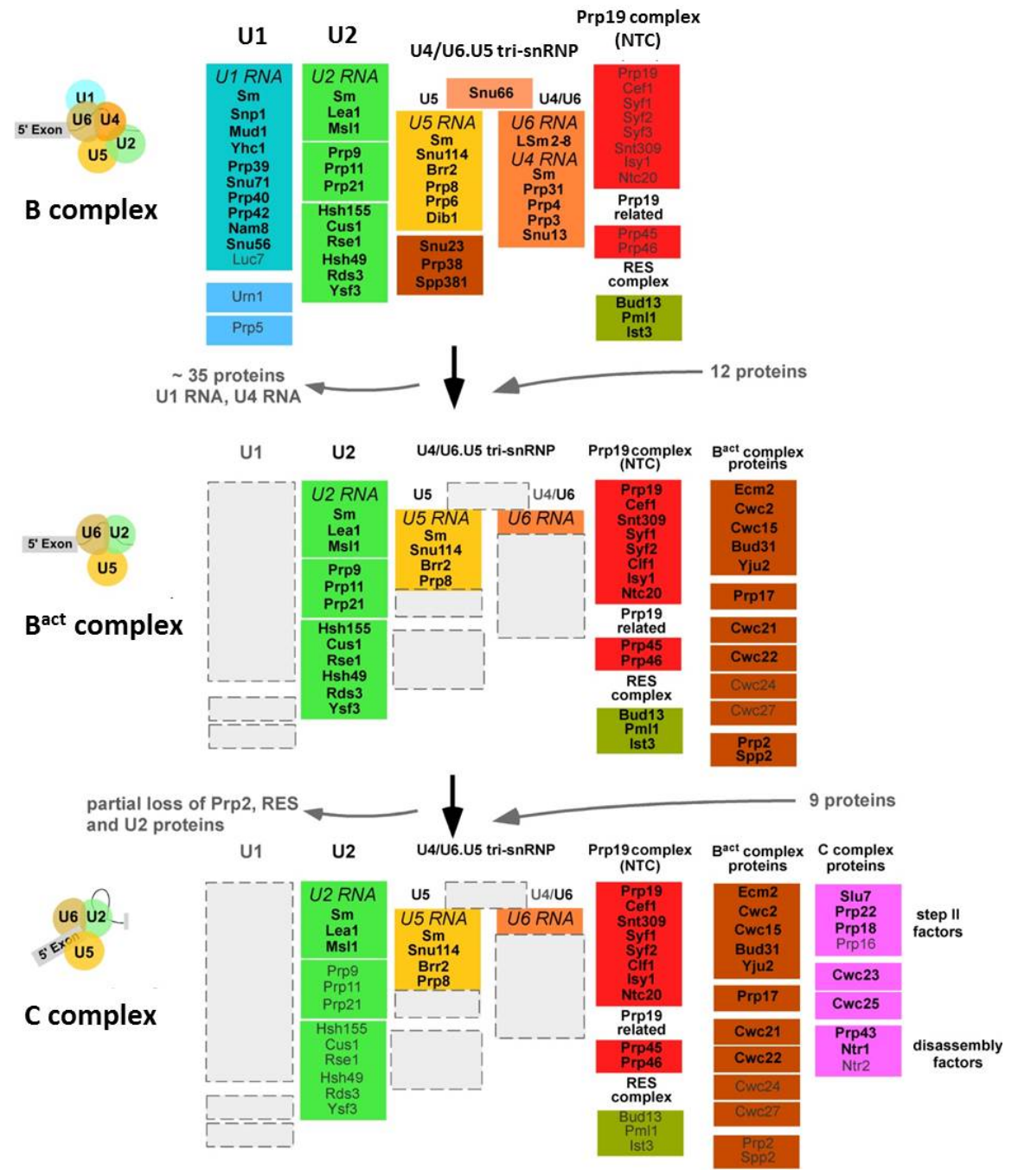

Figure 7: Compositional dynamics of yeast spliceosomes. The snRNP and protein compositions of the $B, B^{\text {act }}$ and $C$ complex are shown. The complexes were analyzed by mass spectrometry. Arrows depict the recruitment and release of different proteins. The NTC complex is shown in red, the $B^{\text {act }}$ complexes in brown and the proteins of the $C$ complex in purple. (Figure adapted from Fabrizio et al., 2009 ${ }^{[37]}$ ) 
After completion of the first catalytic step, the spliceosomal C complex is formed. The compositional dynamics of yeast spliceosomes at the stages of the B, $\mathrm{B}^{\text {act }}$, and $\mathrm{C}$ complexes are summarized in figure 7 .

In the next step, this complex has to be remodeled for the second catalytic step. The activation is achieved by the DEAH-box ATPase Prp16. By the hydrolysis of ATP by Prp16, the 3'SS is rearranged [55 and Slu7, Prp18, and Prp22 are necessary for an efficient second catalytic step. All four proteins, Prp16, Slu7, Prp18, and Prp22, are not present in the $B^{\text {act }}$ complex 48 [46. The function of Slu7 and its heterodimeric complex partner Prp18 is to dock the 3'SS into the active site of the catalytic center [56] [6]. Then the 3' hydroxyl group of the 5' exon can attack the 3'SS and the exons are ligated. After the second catalytic step, the mature RNA is dissociated from the spliceosome induced by the action of the DEAH-box ATPase Prp22. This helicase disrupts the contacts between the mRNA and the U5 snRNP and promotes the release of the mRNA 57 .

After the release of the mRNA, the disassembly of the intron-lariat complex is activated by Prp43 and the spliceosomal factors can be recycled for a new round of splicing. Comparable to Prp2, Prp43 needs a co-factors. This co-factor Ntr1 modulates the weak activity of $\operatorname{Prp} 43[58$.

It was shown, that most of the snRNPs and proteins are recruited to the spliceosome or dissociate from it at stages where they perform their respective functions. These RNP rearrangements involve the transfer of proteins to new interaction partners 59 . All rearrangements in the RNA-RNA network and changes in the protein composition during the splice cycle require driving forces. The DExD/H-box helicases (composed of DEAD-box, DEAH-box and Ski2-like helicases) are involved in these steps by remodeling RNA-RNA, RNA-protein and protein-protein interactions and are shown in table 160] [59.

The most interesting helicases for the present study are Prp2, Prp16, Prp22, and Prp43. These helicases are involved in the activation of the spliceosome for the catalytic steps, the catalytic steps themselves, and the disassembly of the spliceosome.

\subsubsection{The second catalytic step in detail}

Step 2 factors, i.e. Prp16, Slu7, Prp18, and Prp22, are important for the remodeling of the spliceosome for the second catalytic step. Their role in the formation of an active site for the second step and the ligation of the exons is poorly understood. 


\begin{tabular}{ccc} 
Helicase family & ATPase & Function in splice cycle \\
\hline \hline DEAD-box & Sub2 & Pre-spliceosome \\
DEAD-box & Prp5 & Pre-spliceosome \\
DEAD-box & Prp28 & Early activation \\
Ski2-like & Brr2 & Early activation \\
DEAH-box & Prp2 & Late step-1 activation \\
DEAH-box & Prp16 & Step-2 activation \\
DEAH-box & Prp22 & mRNA release \\
DEAH-box & Prp43 & Disassembly
\end{tabular}

Table 1: ATPases of the yeast spliceosome and their role in the splice cycle.

Also, the time points of their recruitment are uncertain. It is known, that the spliceosome is rearranged by the hydrolysis of ATP by Prp16 and because of these rearrangements the 3'SS becomes protected against DNA-directed RNaseH cleavage 55 . It was assumed that Prp16 dissociates from the spliceosome concomitantly with ATP hydrolysis and then the other step 2 factors are recruited 55156 . Further studies suggested, that Slu7 is involved in the selection of the 3'SS because of its possible crosslink to the 3'SS during the second step 61|62.

After the binding of Slu7 and Prp18 to the spliceosome, Prp22 is recruited [56. One of its functions is the release of the mRNA from the spliceosome. This process is ATP-dependent, but Prp22 also has an ATP-independent function, which is the promotion of the second catalytic step 63]64. Immunoprecipitation studies on the recruitment of the second step factors were done with Slu7-, Prp18-, or Prp22-depleted yeast extracts and showed, that after the hydrolysis of ATP by Prp16, first Slu7 is bound, then Prp18, and in the end Prp22. After this ordered recruitment, all three proteins promote the second catalytic step 56 .

Previous depletion/reconstitution studies showed further that the requirement of the second step factors is less strict if the distance between the branch site and the 3'SS is short 65 66 64 [56. Studies on the order of recruitment were based on an actin pre-mRNA substrate with a distance of $38 \mathrm{nt}$ between BS and 3'SS. This distance was then shortened down to $7 \mathrm{nt}$ and a less strict ordered recruitment was observed [66. Because of the use of only whole cell extracts, immunodepletion and immunoprecipitation techniques had to be used. These techniques have limitations which did not enable clear observations of the action mechanism of the step 2 factors during the second step catalysis. 
Until now it is unclear, how the C complex is restructured during the Prp16-mediated catalytic activation. It has been hypothesized that the spliceosome has one singleactive site in the catalytic center $[67$. Because of only one single active site, this site has to be remodeled after the first catalytic step for the second catalytic step. The lariat has to be displaced and the 3'SS has to be in the catalytic center. Then it is well positioned for the nucleophilic attack by the 5' exon. This is a different conformational state than for the first catalytic step. The substrates have to be bound in different ways for both catalytic steps in two distinct conformational states 61 [40. One assumption is that the spliceosome passes through the two different conformations during the catalytic phase and a "two-state model" was proposed [68] (see fig. 8).

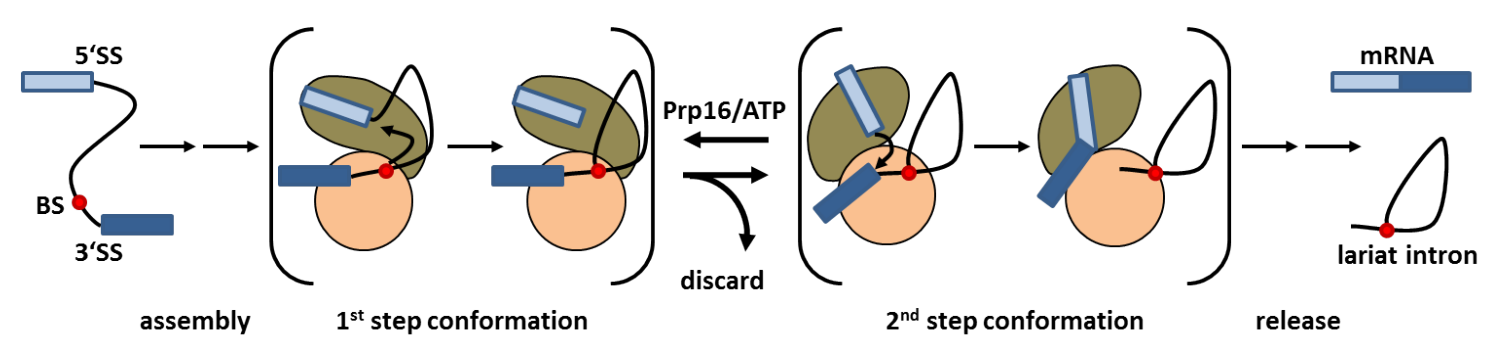

Figure 8: The two-state model for the catalytic steps. Scheme for the proposed two-state model of the conformational states for the first and second catalytic step. It is assumed that the ATPase Prp16 facilitates the transition between the first and second steps. (Figure adapted from Konarska et al., 2005[68]

In the two-state model, the conformational states exist at equilibrium. Studies with mutant alleles in which some mutant alleles improved the first step and inhibited the second one and vice versa showed that the conformational states are competing with each other 68. This two-state model is comparable to the two-state model of the ribosome. The ribosome switches between an open and a closed conformation during the decoding of mRNA. Comparably, the spliceosome may switch between an open and closed conformation during the catalytic steps. In this case, the transitions are supported by proteins and helicases, i.e. the helicases Prp2 and Prp16 may facilitate the transitions 68. An open question is whether the structural change during the transition is accompanied by remodeling events comparable to the remodeling of the $\mathrm{B}^{\text {act }}$ complex by the Prp2-mediated catalytic activation 54. It was shown, that by the action of Prp16, two proteins, Cwc25 and Yju2, are displaced ${ }^{69}$. It is unclear whether the action of additional step 2 factors is necessary for their displacement and whether the formation of the active site for the second catalytic step requires 
only Prp16 or another step 2 factor. Further step 2 factors could help to form the active site or stabilize the step 2 conformation. These factors could be Cwc25, Slu7, Prp18, and Prp22. The question about the proteins necessary for the step 2 conformation is poorly answered until now. The assumption that the docking of the 3'SS into the step 2 active site may stabilize the catalytic center for step 2 catalysis has to be tested.

In this study, the role of the step 2 factors in the splicing of yeast pre-mRNA is investigated and a splicing system in which the spliceosomal complexes are stalled at the $\mathrm{B}^{\text {act }}$ stage was used. This complex can be complemented with recombinant proteins, i.e. step 1 and step 2 factors, to activate the complex for the catalytic steps and to let it run through the catalytic steps.

The binding behavior of the following proteins was observed with FCCS:

$\begin{array}{cl}\text { Snu114 } & \text { U5snRNP protein } \\ \text { Cwc25 } & \text { Step 1 splicing factor } \\ \text { Prp16 } & \text { Step } 2 \text { splicing factor } \\ \text { Slu7 } & \text { Step } 2 \text { splicing factor }\end{array}$

Their binding to the $\mathrm{B}^{\text {act }}, \mathrm{B}^{*}, \mathrm{C}, \mathrm{C}^{*}$, and $\mathrm{C} 2 \mathrm{nd}$ complex was investigated to analyze their recruitment order to the spliceosome. The spliceosomal complexes under investigation are shown in figure 9 .

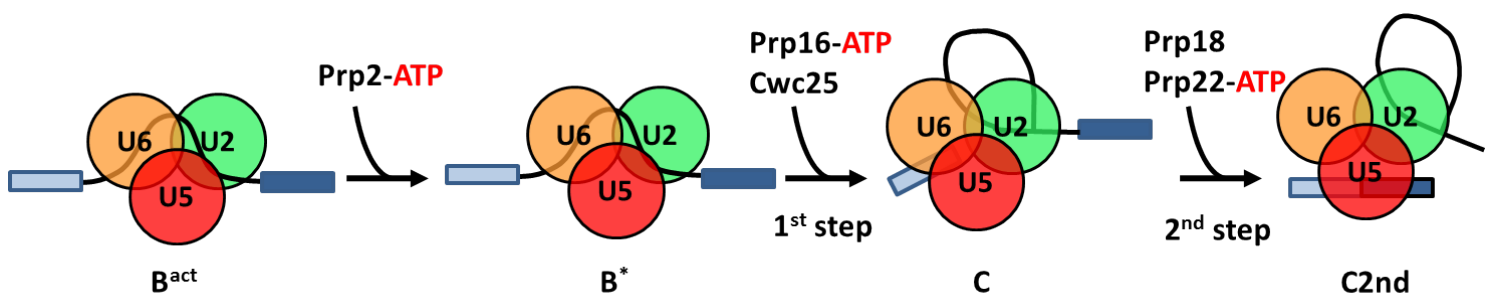

Figure 9: Spliceosomal complexes to which the binding of several proteins was observed with FCCS.

The binding of Cwc25, i.e. a step 1 factor and the following questions were investigated: when is Cwc25 bound to the spliceosomal complexes? When is it released? How does it bind to different pre-mRNA substrates? What is its role in the catalytic steps? By using different pre-mRNA substrates, i.e. with a longer distance between BS and 3'SS and with a shorter distance between these, differences in the recruitment of the step 2 factors can be observed. The minimal requirements of proteins 
for the second step can be defined. The question, whether Slu7 and Prp16 have the same binding site on the spliceosome or not is investigated. 


\section{Materials and Methods}

\subsection{Dual-Color Fluorescence Cross-Correlation Spectroscopy}

Dual-Color Fluorescence Cross-Correlation Spectroscopy (FCCS) is a fluorescence spectroscopy method based on Fluorescence Correlation Spectroscopy (FCS $)$. FCS is a sensitive, optical method which provides information about molecules by analyzing fluctuations in a fluorescence signal.

The experimental setup of FCS is based on a confocal microscope. Its key feature is the diffraction-limited excitation of a fluorescent sample and confocal detection (see fig. 10). In general, the excitation light is focused by an objective into the sample solution. The fluorescence light is then collected by the same objective and passed through a pinhole. Only fluorescence light generated very close to the focal plane can pass through the pinhole, whereas out-of-focus light is blocked. Combining focused illumination and a pinhole, only light from a very small volume is detected by the photo detector. This detection volume is described by a convolution of the volume created by the focused laser beam and the detection system including the objective, the pinhole, any lens, or filter. It is basically an region in solution in which molecules are fluorescently excited and fluorescence detection takes place. The shape of the detection volume is described by a three-dimensional Gaussian ellipsoid with a few hundred nanometers in focus diameter and one to two micrometer along the optical axis $[70$. The exact shape of the detection volume depends on the quality of the optical elements and can be changed by several parameters, e.g. coverslide thickness or laser intensity 13171.

In an FCS experiment, single molecules carrying a fluorophore diffuse through the detection volume. Typically, the emitted photons are detected by single-photon avalanche diodes and the time-dependent intensity fluctuations are analyzed by a temporal auto-correlation analysis of the fluorescence signal. From this analysis the translational and rotational diffusion of a fluorescent molecule can be determined. Additionally, information about the average number of molecules in the observation volume and triplet state dynamics of the fluorophores are accessible 13171.

FCS can be used for determining diffusion coefficients but has only limited accuracy in observing binding reactions. For observing binding interactions FCCS can be used. 


\subsubsection{Principle of FCCS}

In FCCS, two species of molecules are labeled with two different fluorophores with separated excitation and emission spectra. A typical FCCS setup is shown in figure 10.

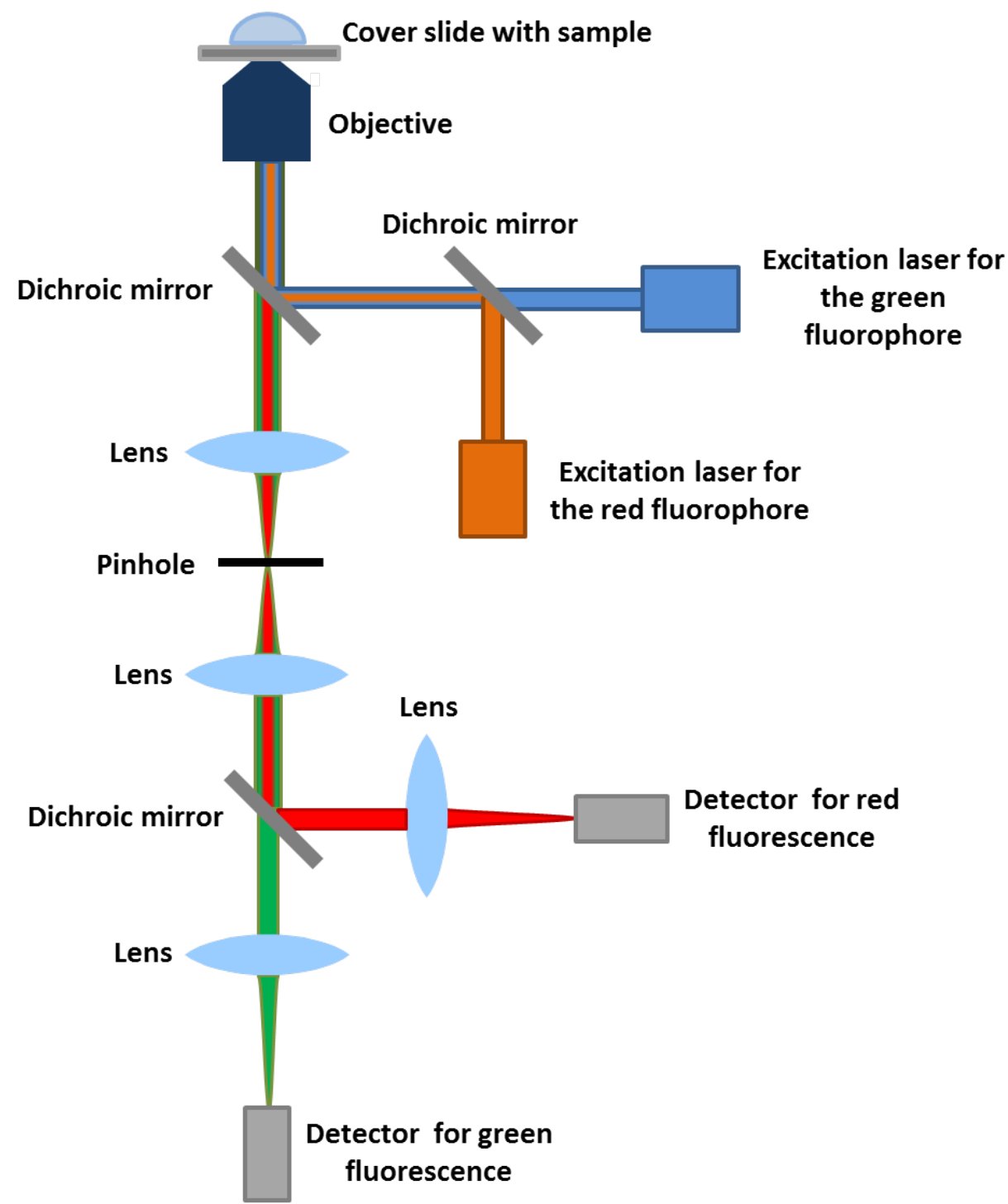

Figure 10: Confocal microscope setup for Dual-Color Fluorescence CrossCorrelation Spectroscopy. A green and red laser beam are combined and injected into a confocal microscope. The fluorescent light of two fluorophores with separated emission spectra is spectrally resolved and detection is done by single photon detectors.

The sample is excited by a green and red laser. The beams of both lasers are combined and reflected into the objective by a dichroic mirror. The objective then focuses the beams into the sample solution. Thereby, two overlapping confocal vol- 
umes of different colors are generated. The fluorophores are excited and the emitted light passes through the objective, the dichroic mirror, and a pinhole. The different fluorescence signals are separated according to their wavelength by a second dichroic mirror. Detection is done by photon detectors in a spectrally resolved manner for the red and green fluorescence signals.

In principle, there are three different types of molecules in the sample: molecules labeled with a fluorophore with a shorter excitation wavelength (hereafter denoted as green labeled molecules or g), molecules labeled with a fluorophore with a longer excitation wavelength (hereafter denoted as red labeled molecules or r), and complexes consisting of both molecules and carrying both fluorophores (hereafter denoted as green/red complexes or gr). For each detection channel the fluorescence signals over time of the different species can be monitored and the time-dependent fluctuations can be observed in each channel (figure 11).

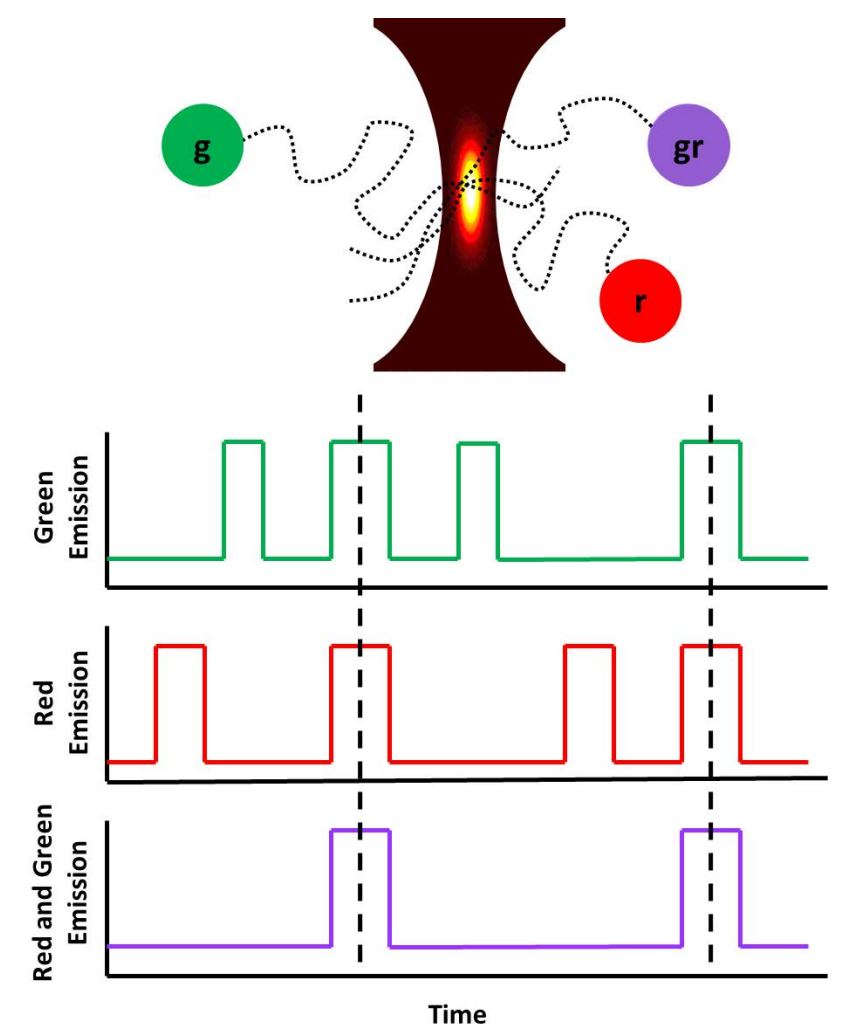

Figure 11: Detection principle of Dual-Color Fluorescence Cross-Correlation Spectroscopy (adapted from Lakowicz ${ }^{[13]}$ ).

If a green labeled molecule diffuses through the detection volume, there is only a signal in the green detection channel. The same applies for the red labeled molecule. If the green/red labeled complex, which consists of the green and the red labeled molecule, diffuses into the volume, a signal is observed in both channels (the green 
one and the red one). To analyze the binding behavior of the green labeled molecule to the red labeled molecule, the cross-correlation between both signals is calculated. If the molecules are not bound and diffuse separately, the fluorescence signals are uncorrelated. Only the signals from bound molecules, which move together through the confocal volume, are correlated. Calculating the cross-correlation between both fluorescence signals yields information about the binding behavior between the two molecules. The cross-correlation amplitude is directly proportional to the number of molecules which carry both fluorophores, the green one and the red one. A low cross-correlation amplitude corresponds to a low binding affinity between the molecules and a high cross-correlation amplitude reflects a high binding affinity of the molecules. An example for the cross-correlation amplitudes is given in figure 12. There, a green labeled molecule interacts with a red labeled molecule. If the red labeled molecule is bound to the green labeled molecule, the cross-correlation amplitude is large. If the red labeled molecule is released, the cross-correlation amplitude is small.
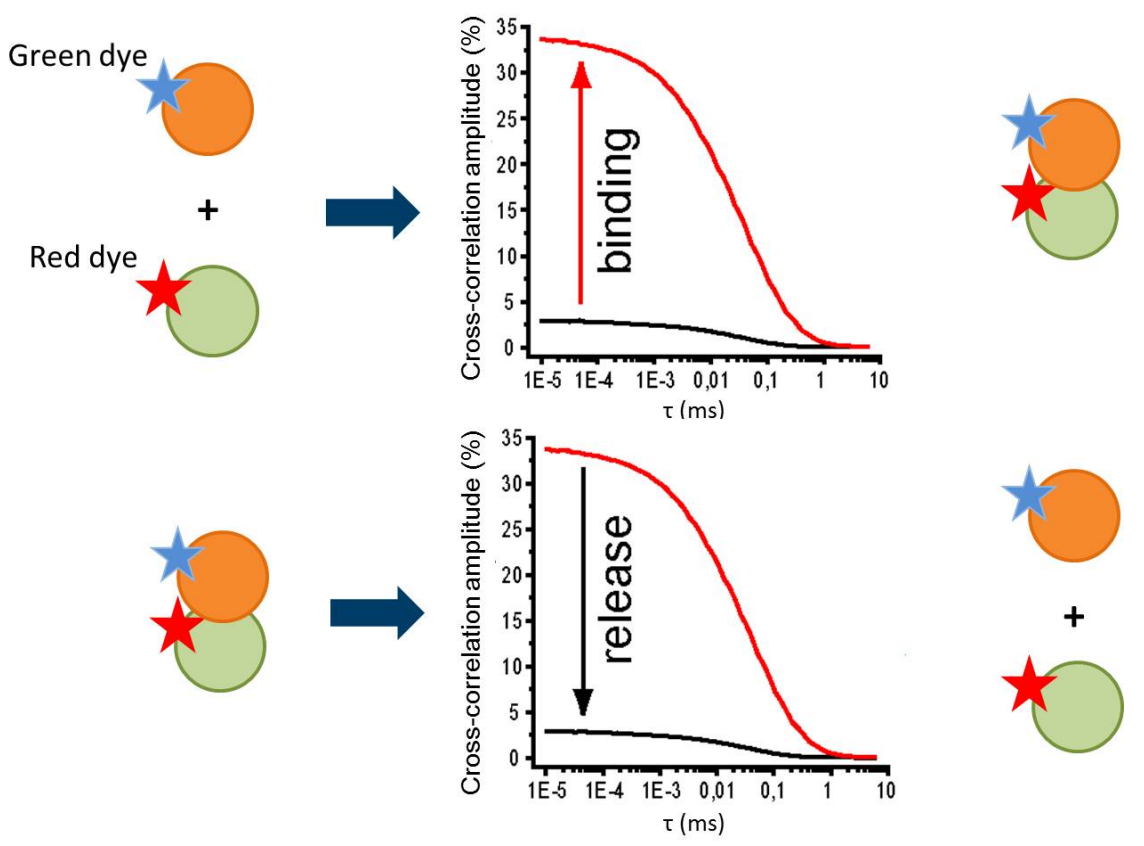

Figure 12: Principle of FCCS: A high cross-correlation amplitude corresponds to the binding of one molecule to another one and a low cross-correlation amplitude reflects the release of one molecule from the other one. 


\subsubsection{Theoretical background of FCCS}

In FCCS, the fluorescence intensities $I_{g}$ and $I_{r}$ of the fluorophores are detected in different channels. For these intensities, the Auto-Correlation Function (ACF) for the green $(\mathrm{g})$ and red (r) channel, respectively, can be calculated:

$$
\begin{aligned}
& g_{g}(\tau)=\left\langle I_{g}(t) \cdot I_{g}(t+\tau)\right\rangle_{t} \\
& g_{r}(\tau)=\left\langle I_{r}(t) \cdot I_{r}(t+\tau)\right\rangle_{t}
\end{aligned}
$$

$I(t)$ is the fluorescence intensity at time $t$ and $I(t+\tau)$ is the intensity at time $t+\tau$ with a time shift (lag time) $\tau$ between the two signals.

The ACF is directly proportional to the probability to detect a photon at time $\tau$ if there was a photon detection event at time zero. This probability consists of two terms: The first term contains the correlated signals and the photons from the same molecule. These photons are physically correlated. The second term results from uncorrelated signals, i.e. from photons which were emitted by different molecules. The backscattered laser light also contributes to this term. The uncorrelated signals have no physical correlation but contribute to a constant offset of the ACF.

In FCS and FCCS, the correlation functions are usually normalized and the intensity fluctuations $\delta I(t)$

$$
\delta I(t)=I(t)-\langle I(t)\rangle
$$

are considered. The time-averaged intensity is denoted as

$$
\langle I(t)\rangle=\frac{1}{T} \int_{0}^{T} I(t) \mathrm{dt}
$$

and the normalized correlation functions are given by

$$
\begin{aligned}
& G_{g}=\frac{\left\langle I_{g}(t) \cdot I_{g}(t+\tau)\right\rangle}{\left\langle I_{g}(t)\right\rangle^{2}}-1=\frac{\left\langle\delta I_{g}(t) \cdot \delta I_{g}(t+\tau)\right\rangle}{\left\langle I_{g}(t)\right\rangle^{2}} \\
& G_{r}=\frac{\left\langle I_{r}(t) \cdot I_{r}(t+\tau)\right\rangle}{\left\langle I_{r}(t)\right\rangle^{2}}-1=\frac{\left\langle\delta I_{r}(t) \cdot \delta I_{r}(t+\tau)\right\rangle}{\left\langle I_{r}(t)\right\rangle^{2}}
\end{aligned}
$$

Figure 13 shows an example for an auto-correlation curve. For small lag times $\tau$, the amplitude of the ACF is high because the molecule with the fluorophore is still in the confocal volume. A photon emitted at time $t$ will be also emitted from the same fluorophore at time $t+\tau$. If the molecule diffuses out of the confocal volume at higher lag times, the possibility to detect two photons from the same fluorophore will be reduced. The curve decays to zero. 


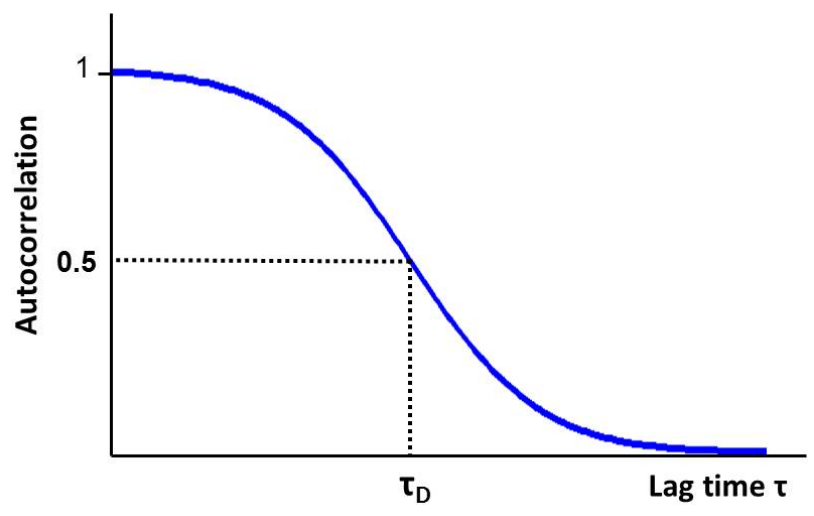

Figure 13: Example of an auto-correlation curve with the diffusion time $\tau_{D}$.

By calculating the ACFs of the green and the red signal, information about the temporal fluctuations of the fluorescence signal of the green and red molecules are obtained. From this data, the diffusion coefficient $D$ of the molecules can be determined. The diffusion time $\tau_{D}$ of a molecule is defined as the average residence time of the dye in the confocal volume. It has to be kept in mind that the ACF of one color arises from two contributions: the fluorescence signal of free, i.e. green labeled molecules, and green labeled molecules which are bound to red labeled molecules. To observe the diffusion coefficient of a green/red complex as well as the amount of green/red complexes, the Cross-Correlation Function ( $\mathrm{CCF}$ ) has to be calculated. The Cross-Correlation Function between two spectrally distinct signals is given by:

$$
g_{g r}=g_{r g}=\left\langle I_{g}(t) \cdot I_{r}(t+\tau)\right\rangle
$$

The normalized Cross-Correlation Function is:

$$
G_{g r}=\frac{\left\langle I_{g}(t) \cdot I_{r}(t+\tau)\right\rangle}{\left\langle I_{g}(t)\right\rangle\left\langle I_{r}(t)\right\rangle}-1=\frac{\left\langle\delta I_{g}(t) \cdot \delta I_{r}(t+\tau)\right\rangle}{\left\langle I_{g}(t)\right\rangle\left\langle I_{r}(t)\right\rangle}
$$

The calculation of a CCF is similar to an ACF, except that the photons from different detection channels are correlated and only photons from molecules which carry both fluorophores ( $\mathrm{g}$ and $\mathrm{r}$ ) are contributing to the CCF. Fluorescence signals from molecules which do not form complexes but are diffusing through the confocal volume at the same time are uncorrelated. They contribute to the offset of the CCF. To describe the detected fluorescence signal in an FCCS experiment, the different fluorescently labeled molecules have to be considered. A general approach is to assume $n$ different fluorescent species. The fluorescence signal is then given by the 
sum of the intensities of the species $i=1 \ldots n$ :

$$
I(t)=\sum_{i=1}^{n} \beta_{i} \epsilon_{e x, i} \phi_{i} \int_{V} I_{e x}(\vec{r}) S(\vec{r}) C_{i}(\vec{r}, t) d^{3} \vec{r}
$$

with the detection efficiency $\beta_{i}$, the extinction coefficient $\epsilon_{e x, i}$, the quantum yield $\phi_{i}$, and the concentration $C_{i}(\vec{r}, t)$ of the fluorescent species $i$. In a typical FCCS experiment, two species are present $(n=2)$.

$I_{e x}(\vec{r})$ is the intensity profile of the excitation laser. $S(\vec{r})$ is the Collection Efficiency Function ( $\mathrm{CEF}$ ). This function describes the fraction of photons which are emitted by a point source, pass through the pinhole, and are seen by the detector. The value of $S(\vec{r})$ depends on the location and size of the image of a point emitter with respect to the pinhole 72 737 74 . The CEF is also connected to the Molecular Detection Function (MDF). This is the CEF multiplied by the excitation intensity $I_{e x}$ and divided by the amplitude of the excitation intensity $I_{0}$ :

$$
W(\vec{r})=\frac{I_{e x}(\vec{r})}{I_{0}} S(\vec{r})
$$

Important parameters for the Molecular Detection Function are the spatial dimensions of the focused laser beam, the laser beam intensity, and the CEF. The MDF takes into account the excitation probability and how a point emitter is imaged by an optical system.

The spatial distribution of the MDF $W(\vec{r})$ is approximated by a three-dimensional Gaussian model [73] and describes the detection volume:

$$
W(\vec{r})=\exp \left(-\frac{2\left(x^{2}+y^{2}\right)}{\omega_{0}^{2}}-\frac{2 z^{2}}{z_{0}^{2}}\right)
$$

with the lateral radius of the detection volume $\omega_{0} . \omega_{0}$ is defined as the radius at which the intensity of the laser has decreased to a value of $1 / \mathrm{e}^{2} .2 z_{0}$ is the axial length of the detection volume.

Substituting the definition of the molecular brightness $\left(\eta_{i}=\beta_{i} \epsilon_{e x, i} \phi_{i} I_{0}\right)$ and the MDF (eq. 10) into eq. 9, the fluorescence signal can be described as

$$
I(t)=\sum_{i=1}^{n} \int_{V} W(\vec{r}) \eta_{i} C_{i}(\vec{r}, t) d^{3} \vec{r}
$$

and the intensity fluctuations as

$$
\delta I(t)=\sum_{i=1}^{n} \int_{V} W(\vec{r}) \delta\left(\eta_{i} C_{i}(\vec{r}, t)\right) d^{3} \vec{r}
$$


By inserting eq. 13 in the formula for the auto-correlation function (eq. 5), the correlation curves can be derived. For the case of only one labeled species $(n=1)$, the ACF is given by

$$
G(\tau)=\frac{\int_{V^{\prime}} \int_{V} W(\vec{r}) W\left(\vec{r}^{\prime}\right)\left\langle\delta(\eta C(\vec{r}, t)) \delta\left(\eta C\left(\vec{r}^{\prime}, t+\tau\right)\right)\right\rangle d^{3} \vec{r} d^{3} \vec{r}^{\prime}}{\left(\int_{V} W(\vec{r})\langle\eta C(\vec{r}, t)\rangle d^{3} \vec{r}\right)^{2}}
$$

The fluctuation term can be converted into

$$
\delta(\eta C(\vec{r}, t))=C(\vec{r}, t) \delta \eta+\eta \delta C(\vec{r}, t)=\eta \delta C(\vec{r}, t)
$$

with the constant brightness $\delta \eta=0$.

In the next step the effective volume $V_{\text {eff }}$ is defined

$$
V_{e f f}=\frac{\left(\int_{V} W(\vec{r}) d^{3} \vec{r}\right)^{2}}{\int_{V}(W(\vec{r}))^{2} d^{3} \vec{r}}=\pi^{3 / 2} \omega_{0}^{2} z_{0}
$$

Further, the concentration correlation function $\Phi$ is defined as

$$
\Phi\left(\vec{r}, \vec{r}^{\prime}, \tau\right)=\left\langle\delta C(\vec{r}, t) \delta C\left(\vec{r}^{\prime}, t+\tau\right)\right\rangle
$$

For a translationally diffusing molecule without internal dynamics the concentration correlation function 71 is given by

$$
\Phi\left(\vec{r}, \vec{r}^{\prime}, \tau\right)=\frac{\langle C(\vec{r}, t)\rangle}{(4 \pi D \tau)^{3 / 2}} \exp \left(-\frac{\left(\vec{r}-\vec{r}^{\prime}\right)^{2}}{4 D \tau}\right) .
$$

This equation is the solution of the diffusion equation. Equation 18 includes the probability to find a molecule at position $\vec{r}^{\prime}$ at time $\tau$ which was at position $\vec{r}$ at time zero. This probability is multiplied with the average concentration and $D$ is the diffusion coefficient of the molecule. With the concentration correlation function, the auto-correlation can be written as

$$
\begin{aligned}
G(\tau) & =\frac{\int_{V^{\prime}} \int_{V} W(\vec{r}) W\left(\vec{r}^{\prime}\right) \Phi\left(\vec{r}, \vec{r}^{\prime}, \tau\right) d^{3} \vec{r} d^{3} \vec{r}^{\prime}}{\left(\int_{V} W(\vec{r})\langle C(\vec{r}, t)\rangle d^{3} \vec{r}\right)^{2}} \\
& =\frac{1}{\langle C(\vec{r}, t)\rangle V_{e f f}} \frac{1}{1+\frac{4 D \tau}{\omega_{0}^{2}}} \frac{1}{\sqrt{1+\frac{4 D \tau}{z_{0}^{2}}}} \\
& =\frac{1}{\langle N\rangle} \frac{1}{1+\frac{\tau}{\tau_{D}}} \frac{1}{\sqrt{1+\frac{\tau}{a^{2} \tau_{D}}}}
\end{aligned}
$$

With the mean particle number $\langle N\rangle=\operatorname{Var}(N)=\left\langle\delta N^{2}\right\rangle=\langle C(\vec{r}, t)\rangle V_{\text {eff }}$, the diffusion time $\tau_{D}=\frac{\omega_{0}^{2}}{4 D}$, and the parameter $a=z_{0} / \omega_{0}$. 
For a lag time $\tau=0$, considering only one species $(n=1)$ and a fluorophore which is not in the triplet state, and the case that the fluorescence signal is proportional to the concentration, i.e. to the mean particle number, the auto-correlation function is given by

$$
G(0)=\frac{\left\langle\delta I(t)^{2}\right\rangle}{\langle I(t)\rangle^{2}}=\frac{\left\langle\delta N^{2}\right\rangle}{\langle N\rangle^{2}}=\frac{1}{\langle N\rangle}
$$

Equation 22 shows that the amplitude of the normalized auto-correlation curve at lag time zero and the average number of molecules in the detection volume are inversely proportional. It has to kept in mind that photophysical effects can change the molecular brightness and the fluorescence intensity fluctuations. These changes can influence the correlation curve 23.

The number of molecules $N$ depends on the size of the detection volume and on the concentration of the molecules 20 . $N$ varies with the wavelength $\lambda$ of the excitation beam which can be seen by the following relation

$$
\frac{\omega_{g}}{\omega_{r}}=\frac{z_{g}}{z_{r}}=\frac{\lambda_{g}}{\lambda_{r}} .
$$

Consequently, even for equal concentrations of both species, the number of molecules in the detection volumes for both excitation wavelength will be different.

For an analysis of FCCS data, all equations have to be formed for both autocorrelation functions $\left(G_{g}(\tau)\right.$ and $\left.G_{r}(\tau)\right)$ and for the cross-correlation function $\left(G_{g r}(\tau)\right)$. The intensity fluctuations are given by

$$
\begin{aligned}
& \delta I_{g}(t)=\int_{V} W_{g}(\vec{r}) \eta_{g} \delta\left(C_{g}(\vec{r}, t)+C_{g r}(\vec{r}, t)\right) d^{3} \vec{r} \\
& \delta I_{r}(t)=\int_{V} W_{r}(\vec{r}) \eta_{r} \delta\left(C_{r}(\vec{r}, t)+C_{g r}(\vec{r}, t)\right) d^{3} \vec{r}
\end{aligned}
$$

Assuming that the detection volumes of both colors (green and red) are ideally overlapping and with equation 21, the correlation functions are

$$
\begin{aligned}
G_{g}(\tau) & =\frac{\left\langle C_{g}\right\rangle K_{g}(\tau)+\left\langle C_{g r}\right\rangle K_{g r}(\tau)}{\left(\left\langle C_{g}\right\rangle+\left\langle C_{g r}\right\rangle\right)^{2} V_{e f f}} \\
G_{r}(\tau) & =\frac{\left\langle C_{r}\right\rangle K_{r}(\tau)+\left\langle C_{g r}\right\rangle K_{g r}(\tau)}{\left(\left\langle C_{r}\right\rangle+\left\langle C_{g r}\right\rangle\right)^{2} V_{e f f}} \\
G_{g r}(\tau) & =\frac{\left\langle C_{g r}\right\rangle K_{g r}(\tau)}{\left(\left\langle C_{g}\right\rangle+\left\langle C_{g r}\right\rangle\right)\left(\left\langle C_{r}\right\rangle+\left\langle C_{g r}\right\rangle\right) V_{e f f}}
\end{aligned}
$$


with the parameter $K_{i}$ where $i$ can take the values $g$, $r$, or $g r$

$$
K_{i}(\tau)=\frac{1}{1+\frac{\tau}{\tau_{D, i}}} \frac{1}{\sqrt{1+\frac{\tau}{a^{2} \tau_{D, i}}}} .
$$

The amplitudes of the correlation curves at lag time zero are

$$
\begin{aligned}
G_{g}(0) & =\frac{1}{\left(\left\langle C_{g}\right\rangle+\left\langle C_{g r}\right\rangle\right) V_{\text {eff }}} \\
G_{r}(0) & =\frac{1}{\left(\left\langle C_{r}\right\rangle+\left\langle C_{g r}\right\rangle\right) V_{\text {eff }}} \\
G_{g r}(0) & =\frac{\left\langle C_{g r}\right\rangle}{\left(\left\langle C_{g}\right\rangle+\left\langle C_{g r}\right\rangle\right)\left(\left\langle C_{r}\right\rangle+\left\langle C_{g r}\right\rangle\right) V_{\text {eff }}}
\end{aligned}
$$

Substituting $\langle N\rangle=\langle C(\vec{r}, t)\rangle V_{\text {eff }}$, the correlation functions are coupled to the particle number in the detection volume by the following relation

$$
\begin{aligned}
G_{g}(0) & =\frac{1}{\left\langle N_{g}\right\rangle+\left\langle N_{g r}\right\rangle} \\
G_{r}(0) & =\frac{1}{\left\langle N_{r}\right\rangle+\left\langle N_{g r}\right\rangle} \\
G_{g r}(0) & =\frac{\left\langle N_{g r}\right\rangle}{\left(\left\langle N_{g}\right\rangle+\left\langle N_{g r}\right\rangle\right)\left(\left\langle N_{r}\right\rangle+\left\langle N_{g r}\right\rangle\right)}
\end{aligned}
$$

These equations show the relation between the amplitudes of the correlation functions and the number of particles in the detection volumes. Comparison of the numerators of the equations 33 - 35 shows that for $N_{g r} \gg N_{g}, N_{r}$ the amplitudes of the auto-correlation functions are always higher or equal to the amplitude of the cross-correlation function 19 . The cross-correlation amplitude $G_{g r}(0)$ is directly proportional to the number of particles which carry both labels, because the denominator in equation 35 is constant in time $\frac{19}{2}$. Changes in the number of double labeled particles $N_{g r}$ caused by binding or dissociation reactions results in changes of the cross-correlation amplitude. These variations can be monitored and allows for determining the binding behavior of molecules. 


\subsubsection{Limitations of FCCS}

While FCCS allows for observation of binding reactions, it has some limitations, with respect to sample preparation, excitation, and detection.

For FCCS measurements the molecules of interest have to be labeled with high labeling efficiency and each molecule has to be labeled with only one fluorophore (1:1 labeling). The molecules of one species should only carry the same fluorophore (e.g. the green fluorophore) and the molecules of the second species another fluorophore (e.g. the red fluorophore). Another limitation is the binding affinity of the molecules. If the binding affinity is too low, a higher concentration of particles is necessary and too many particles are in the detection volume ${ }^{24}$. These are sample related limitations.

Additionally, there are limitations and intrinsic problems on the excitation and detection side 15 . One problem is cross-excitation. The red fluorophore can be excited by the green excitation wavelength (cross-excitation). This cross-excitation can be reduced by choosing dyes with different fluorescence spectra which have only a small overlap. The absorption spectra of the used dyes and the lines of both excitation lasers are shown in figure 14 and illustrate that the green laser can excite the red dye only to a very small extent. Cross-excitation of the green dye by the red laser is negligible by energetic reasons.

A further problem is cross-talk between detection channels. Cross-talk results from green light coupling into the red detection channel and red light coupling into the green channel. These cross-emissions can be suppressed by choosing suitable emission filters. The emission spectra of the used dyes (eGFP, Alexa488, and Atto647N) and transmission spectra of the used emission filters are shown in figure 14. Fig. 14b illustrates that cross-talk resulting from green emission into the red channel is suppressed in our system.

Another way for reducing spectral cross-talk and which is used is Pulsed Interleave Excitation (PIE) [75 25. PIE is based on the use of alternating excitation pulses. The fluorescence emission generated from one pulse has decayed before the next pulse excites the fluorophores. By using interleaved pulses in the sequence of green pulse, red pulse, the excitation source of each detected photon is known and cross-talk is reduced.

Additionally, the two detection volumes have to overlap to excite the two different fluorophores on one green/red complex simultaneously. Due to the different excitation wavelengths, different beam waists, and different divergences of the laser beams, 
the detection volumes have different sizes. This leads to two non-ideally overlapping foci. To involve this factor in the analysis, a sample with $100 \%$ cross-correlation is measured. By assuming $100 \%$ cross-correlation, a correction factor for the detection volumes is calculated.
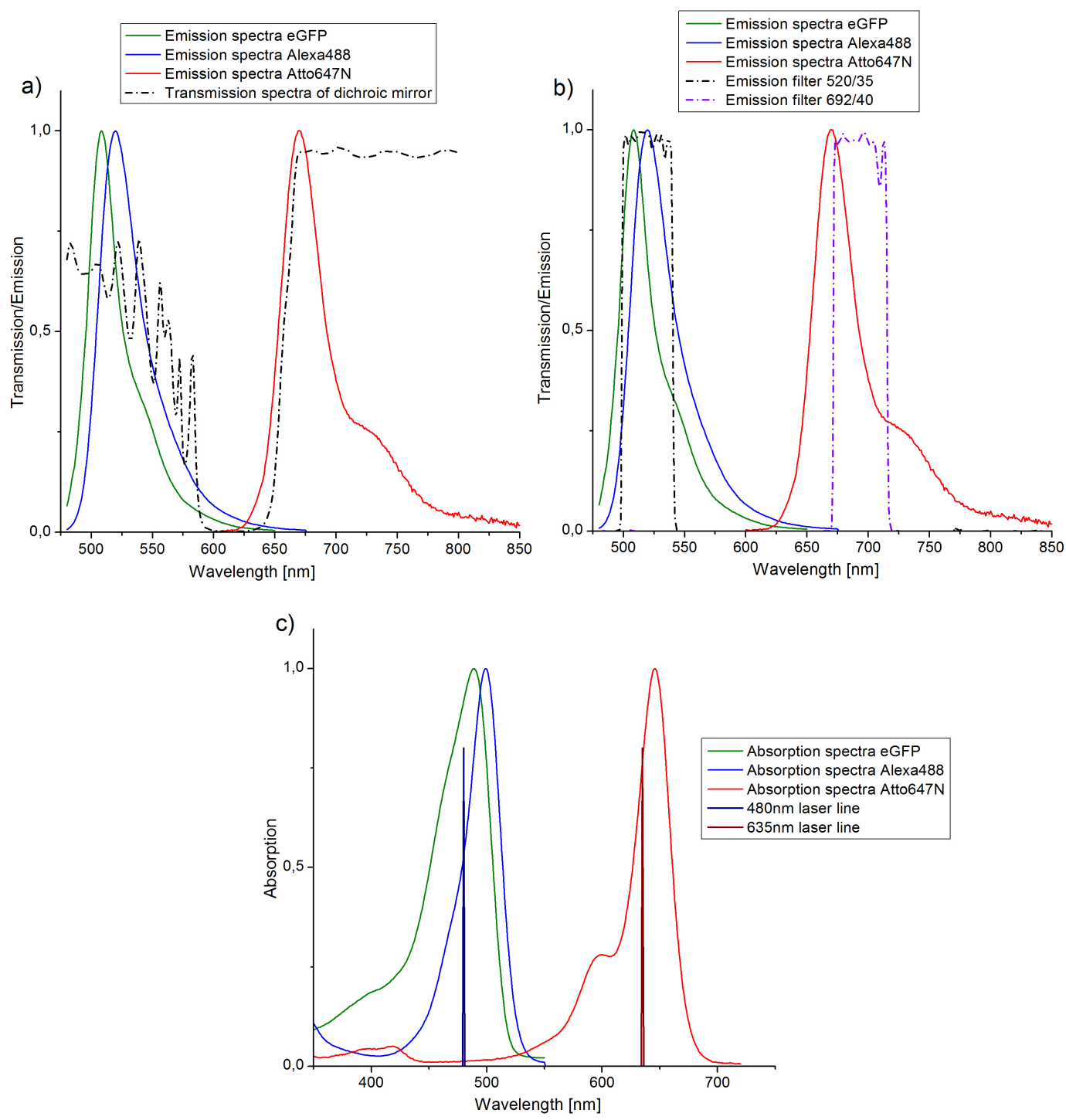

Figure 14: Emission and transmission spectra. a) Emission spectra of the used dyes (blue: Alexa488, green: eGFP, red: ATTO647N) and transmission spectra of the dichroic in front of the objective; b) Emission spectra of the used dyes and transmission spectra of the used emission filters; c) Absorption spectra of the used dyes and lines of the excitation lasers. 


\subsubsection{Dual-Focus Fluorescence Cross-Correlation Spectroscopy}

The setup used for FCCS measurements allows for simultaneously performing DualFocus Fluorescence Correlation Spectroscopy (2fFCS) ${ }^{176}$. 2fFCS is an extension of conventional FCS and allows for determining absolute diffusion coefficients with high precision.

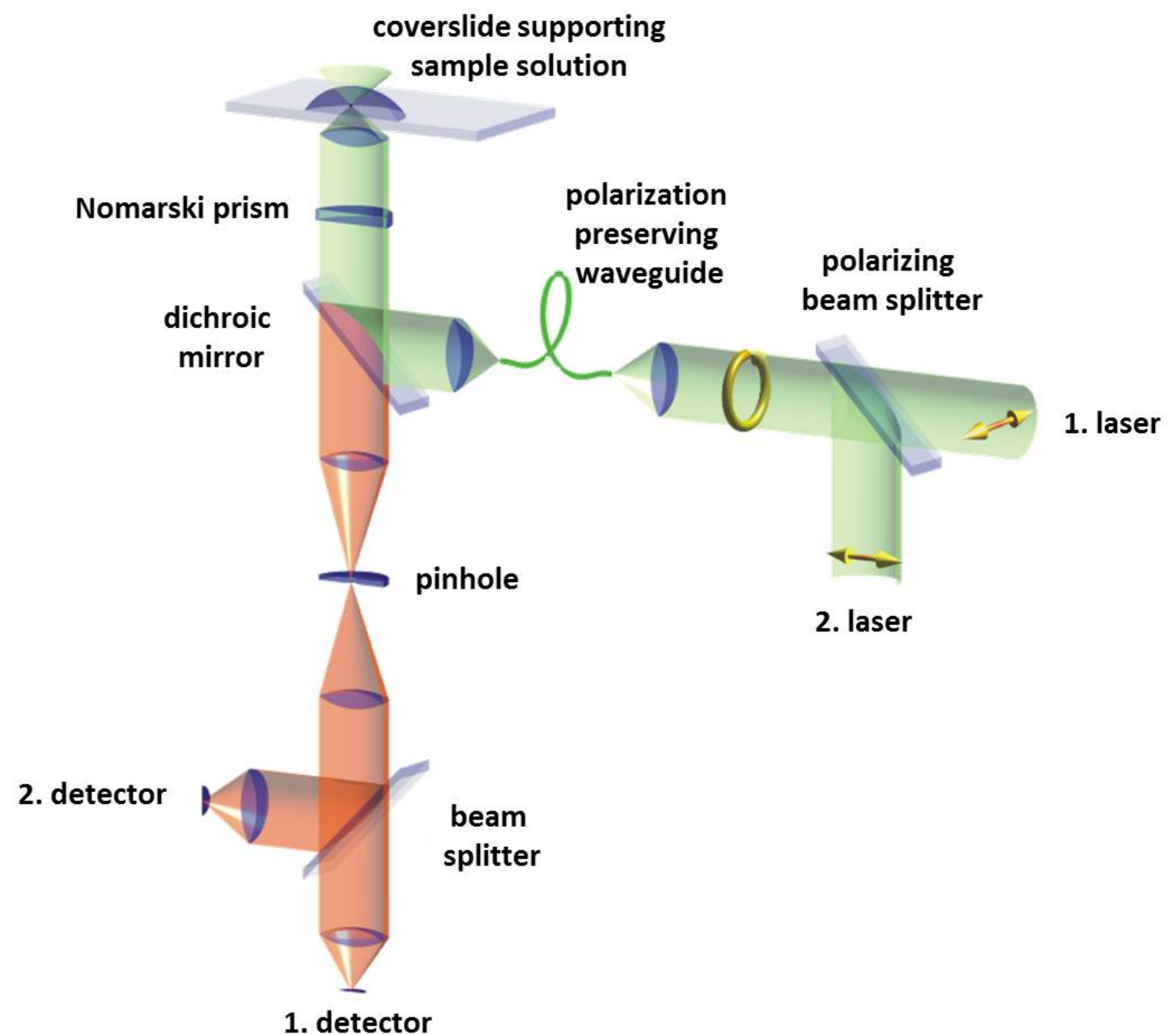

Figure 15: Schematic picture of the 2fFCS setup. Two identical lasers with perpendicular polarization (shown by arrows) are combined and reflected up into a Nomarski prism and the objective. The fluorescence light is collected by the same objective, passes through different optical elements, and is focused onto two detectors (adapted from Dertinger et al. ${ }^{[77}$ ).

In 2fFCS two pulsed excitation lasers with identical wavelength and perpendicular polarization are used. A Nomarski prism is placed in front of the objective. The laser beams are combined and reflected into the Nomarski prism by a dichroic mirror 
(see fig. 15). Due to their perpendicular polarizations, the laser beams are deflected by the Nomarski prism under different angles (shown in fig. 16). Afterwards, they are focused by the objective into the sample solution and two identical but laterally shifted and overlapping foci are generated. The distance $\delta$ between the foci depends on the properties of the Nomarski prism and the wavelength of the excitation beam. For a specific wavelength, the distance is fixed and constitutes an intrinsic ruler to the system. Fluorescence is then collected by the same objective and passed through the Nomarski prism, the dichroic mirror, and the pinhole. After the pinhole, the light is split and focused onto single-photon counting detectors. Using a single-photon counting device which detects the arrival times of each recorded photon allows for determining which fluorescent photon was generated by which laser. Then AutoCorrelation Functions for each focus and the Cross-Correlation Function between both foci can be calculated. Knowledge of the distance $\delta$ allows for calculating absolute values of the diffusion coefficient from the ACFs and

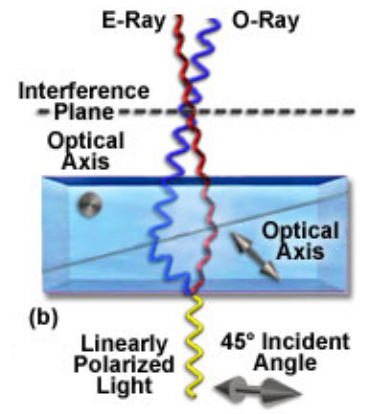

Figure 16: Schematic of a Nomarski prism. The ordinary and extraordinary rays correspond to the laser beams with perpendicular polarization (adapted from www.microscopyu.com) CCF 77 .

The theory of $2 \mathrm{fFCS}$ is similar to the theory of FCCS. In the case of $2 \mathrm{fFCS}$, the signals of the two shifted foci are cross-correlated:

$$
\begin{aligned}
G_{C C, 1}(\tau) & =\frac{\left\langle\delta I_{1}(t) \cdot \delta I_{2}(t+\tau)\right\rangle}{\left\langle I_{1}(t)\right\rangle\left\langle I_{2}(t)\right\rangle} \\
G_{C C, 2}(\tau) & =\frac{\left\langle\delta I_{2}(t) \cdot \delta I_{1}(t+\tau)\right\rangle}{\left\langle I_{1}(t)\right\rangle\left\langle I_{2}(t)\right\rangle}
\end{aligned}
$$

with the intensity signal $I_{1}$ from focus 1 and $I_{2}$ from focus 2 .

In order to obtain accurate diffusion coefficients, an appropiate model function for the MDF is important. This MDF can be approximated by a Gauss-Lorentzian function 76 :

$$
W(\vec{r})=\frac{\kappa(z)}{\omega^{2}(z)} \exp \left(-\frac{2}{\omega^{2}(z)}\left[\left(x \pm \frac{\delta}{2}\right)^{2}+y^{2}\right]\right)
$$


In this model, a Gaussian distribution in each plane perpendicular to the optical axis with the width $\omega(z)$ and the amplitude $\kappa(z) / \omega^{2}(z)$ was approximated. The functions $\omega(z)$ and $\kappa(z)$ are given by

$$
\begin{gathered}
\omega(z)^{2}=\omega_{0}^{2}\left[1+\left(\frac{\lambda_{e x} z}{\pi \omega_{0}^{2} n}\right)^{2}\right] \\
\kappa(z)=1-\exp \left(-\frac{2 \alpha^{2}}{R^{2}(z)}\right)
\end{gathered}
$$

The function $R(z)$ is defined by:

$$
R(z)^{2}=R_{0}^{2}\left[1+\left(\frac{\lambda_{e m} z}{\pi R_{0}^{2} n}\right)^{2}\right]
$$

$\lambda_{e x}$ denotes the excitation wavelength, $\lambda_{e m}$ the emission wavelength, $n$ is the refractive index of the immersion medium, and $\alpha$ is the radius of the confocal aperture defined by the magnification of the system. $\omega_{0}^{2}$ and $R_{0}^{2}$ are unknown model parameters. With the modified MDF, the cross-correlation function between two laterally shifted foci can be written as 76

$G_{C C}(\tau)=\frac{\eta_{1} \eta_{2} C}{4} \sqrt{\frac{\pi}{D \tau}} \int_{-\infty}^{\infty} d z_{1} \int_{-\infty}^{\infty} d z_{2} \frac{\kappa\left(z_{1}\right) \kappa\left(z_{2}\right)}{8 D \tau+\omega^{2}\left(z_{1}\right)+\omega^{2}\left(z_{2}\right)} e^{\left[-\frac{\left(z_{2}-z_{1}\right)^{2}}{4 D \tau}-\frac{2 \delta^{2}}{8 D \tau+\omega^{2}\left(z_{1}\right)+\omega^{2}\left(z_{2}\right)}\right]}$

Here, $\eta_{1,2}$ are two factors which represent the excitation intensities of each focus, the detection efficiencies, and the fluorophores properties.

The obtained diffusion coefficient in an FCS experiment strongly depends on the shape of the detection volume. Variations in the excitation laser intensity, in the coverslide thickness, and in the refractive index of the sample can influence the shape of the observation volume. These problems require a calibration of the system prior to each measurement. This can be overcome by using $2 \mathrm{fFCS}$. The mentioned parameters, e.g. the laser intensity, can change the size and shape of the detection volume of each focus but not the distance between them. Therefore, 2fFCS allows for observing absolute diffusion coefficients without calibration of the setup prior to each measurement. 


\subsubsection{Dual-color dual-focus Fluorescence Cross-Correlation Spectroscopy}

In the used setup, the two methods FCCS and 2fFCS are combined. Combining 2fFCS and FCCS allows for precisely calculating diffusion coefficients while performing highly accurate binding studies. In more detail, for each excitation wavelength (green and red) two lasers with perpendicular polarization are used. By using a Nomarski prism, laterally shifted foci are generated. Thereby, two laterally shifted green foci are overlapping with two laterally shifted red foci. For the calculation of the number of complexes (gr), all Auto-Correlation and Cross-Correlation Functions between the foci and the colors have to be determined (see section 2.1.7).

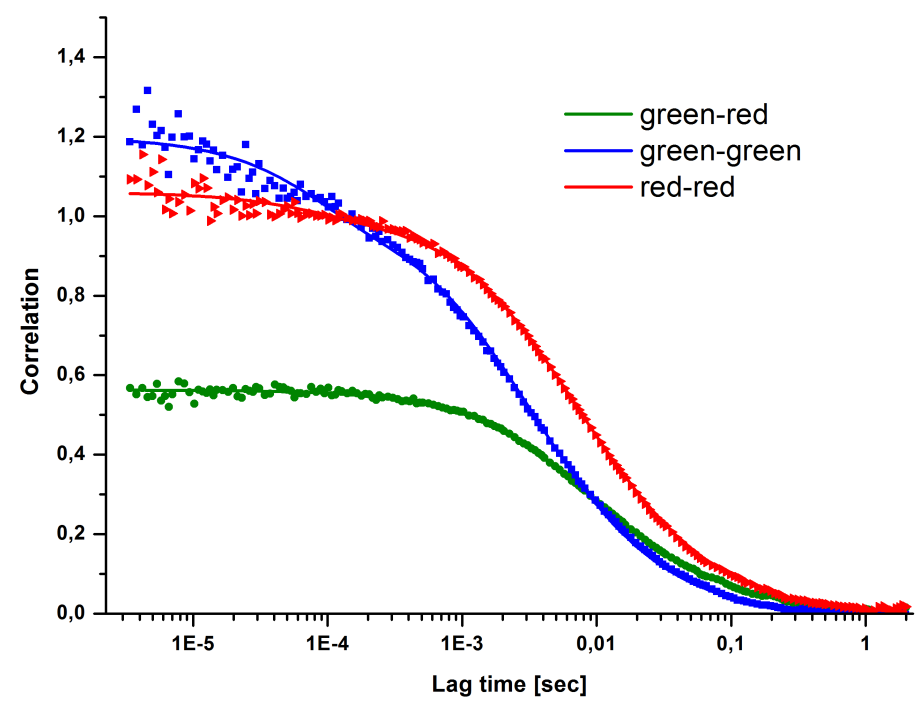

Figure 17: Exemplary representation of correlation curves in an FCCS experiment. The green line and symbols denote the cross-correlation between the two colors, the blue line and symbols the auto-correlation for both green foci and the red line and symbols the auto-correlation for both red foci. The correlation curves are normalized and show the binding of $C_{w c} 24-e G F P$ to $B^{a c t \Delta P r p 2}$.

Typical normalized correlation curves of a dual-color-dual-focus FCCS measurement are shown in figure 17. The amplitude of the Cross-Correlation Function between both colors (green-red) is smaller than the amplitude of both Auto-Correlation Functions of the foci (green-green, red-red). This can be also shown by comparing the amplitudes of the correlation functions (eq. 33 - eq. 35): 


$$
\begin{aligned}
& \frac{G_{g r}(0)}{G_{r}(0)}=\frac{\left\langle N_{g r}\right\rangle}{\left\langle N_{g}\right\rangle+\left\langle N_{g r}\right\rangle} \\
& \frac{G_{g r}(0)}{G_{g}(0)}=\frac{\left\langle N_{g r}\right\rangle}{\left\langle N_{r}\right\rangle+\left\langle N_{g r}\right\rangle}
\end{aligned}
$$

\subsubsection{Used optical setup for the FCCS experiments}

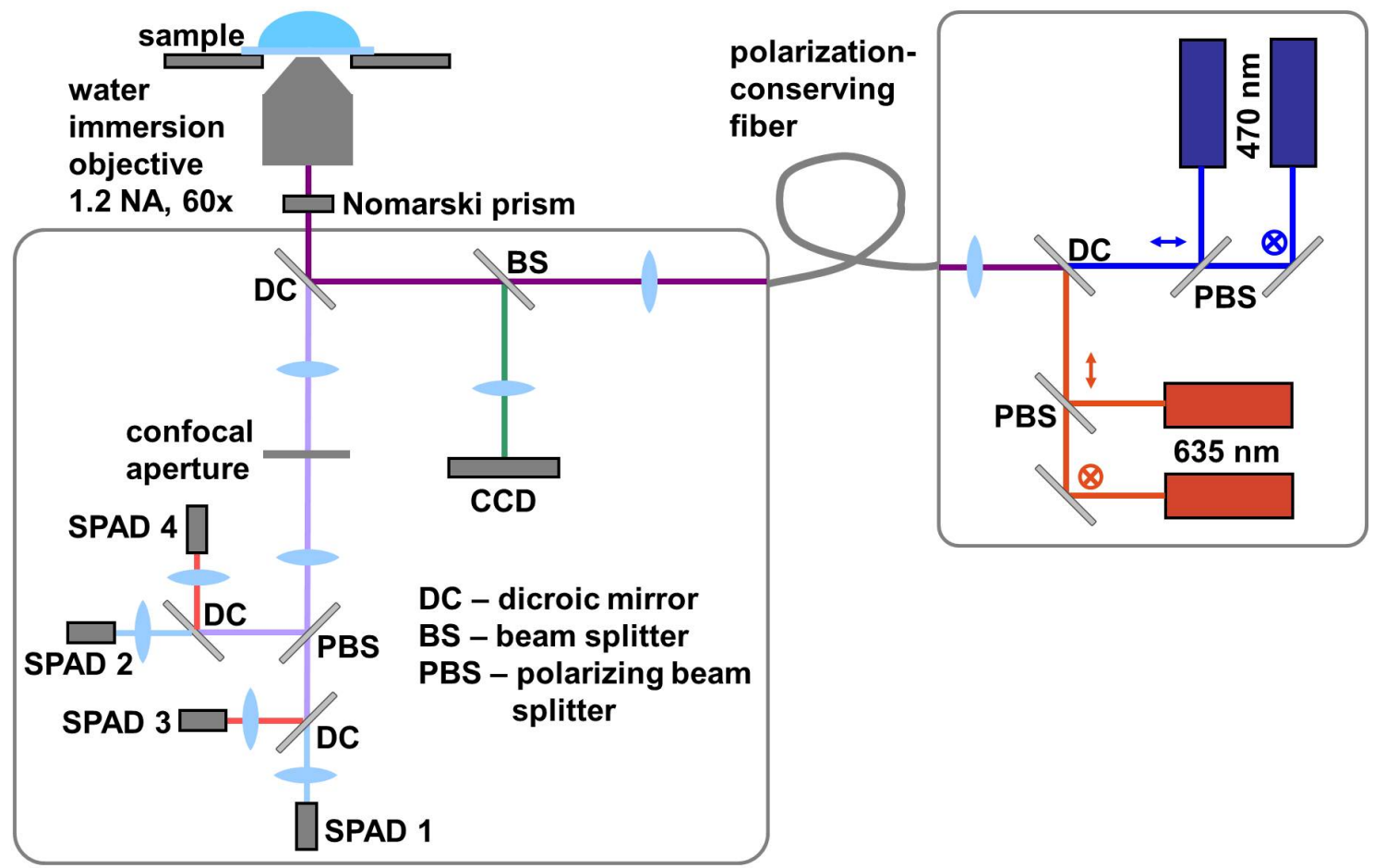

Figure 18: The used optical setup for FCCS and 2fFCS experiments.

The dual-color FCCS setup is based on an inverse epi-fluorescence microscope (MicroTime 200, PicoQuant GmbH, Berlin, Germany) with a 2fFCS extension 76 . The system is equipped with two identically pulsed $480 \mathrm{~nm}$ diode lasers (LDH-P-C-485 B, PicoQuant GmbH, Berlin, Germany) and two identically pulsed $635 \mathrm{~nm}$ diode lasers (LDH-P-635, PicoQuant GmbH, Berlin, Germany) with linear polarization and a pulse duration of $50 \mathrm{ps}$ (FWHM). The lasers are pulsed alternately with an overall repetition rate of $10 \mathrm{MHz}$ (PIE mode) 75 corresponding to a delay of $25 \mathrm{~ns}$ between pulses (PDL 828 "Sepia II", PicoQuant GmbH, Berlin, Germany).

The light of each of the two pairs of identical wavelength lasers is combined by two polarizing beam splitters (Ealing Catalogue, St. Asaph, UK) into single beams. 
These beams are combined by a dichroic mirror (490 dcxr, Chroma Technology, Rockingham, VT, USA) resulting in a single beam containing both wavelengths. The beam is coupled into a polarization-preserving single mode fiber. At the fiber output, the light is collimated and reflected up by a dichroic mirror (Q660LP, Chroma Technology, Rockingham, VT, USA). Before entering the objective, the light is passed through a Nomarski prism (U-DICTHC, Olympus Europa, Hamburg, Germany) which deflects the beam into two slightly different directions depending on their polarization. Then, the light is focused into the microscope's objective (UPLAPO 60x W, 1.2 N.A., Olympus Europa, Hamburg, Germany) and two overlapping foci with a fixed lateral distance determined by the properties of the Nomarski prism are obtained.

Fluorescence is collected by the same objective (epi-fluorescence setup), passed through the Nomarski prism and the dichroic mirror, and focused by a tube lens through a single circular aperture (diameter $150 \mu \mathrm{m}$ ). After the pinhole, the light is re-collimated, split by a polarizing beam splitter cube and a dichroic mirror (640 dcxr, Chroma Technology, Rockingham, VT, USA), and focused onto two single photon avalanche diodes (SPAD, AQR13, Perkin Elmer, Wellesley, MA, USA), respectively, for the red and the blue excitation. Emission bandpass filters HC692/40 and HC520/35 (Semrock, USA) for red and blue excitation, respectively, positioned directly in front of each detector, discriminate fluorescence against scattered light. A single-photon counting electronics (HydraHarp 400, PicoQuant GmbH, Berlin, Germany) independently records the detected photons of all detectors with an absolute temporal resolution of 4 ps on a common time frame.

\subsubsection{Computation and evaluation of the correlation data}

All correlation curves are calculated by a dedicated software 78 . In the first step, the following functions are calculated: the Auto-Correlation Functions (ACFs) of the focal volumes excited by the first green laser (GG1), the second green laser (GG2), the first red laser (RR1), and of the second red laser (RR2). The corresponding Cross-Correlation Functions between both green (GGX) and both red (RRX) focal volumes are also necessary. Here, 1 denotes the focal volume of the first laser, 2 denotes the focal volume of the second laser, and $\mathrm{X}$ denotes the focal volume of the overlap between the volumes of the same color. In the same way, the correlation functions for photon pairs of which the first photon was excited by the first green laser and the second was excited by the first red laser (GR1) are computed. The 
calculation is done accordingly for GR2, RG1, RG2, GRX, and RGX. Since the laser settings and buffer conditions are always constant during one set of experiments, a blank measurement is done using only the buffer as sample. The computation of the correlation data in absolute units of $\mathrm{cps}^{2}$ allows for compensating for the buffer background by simple subtraction of the corresponding blank curves. In this way, the number of data for the subsequent data evaluation can be reduced by setting

$$
\begin{aligned}
G_{n} & =G G_{n}-G G_{n}^{0} \\
R_{n} & =R R_{n}-R R_{n}^{0} \\
M_{n} & =\frac{\left(G R_{n}-G R_{n}^{0}+R G_{n}-R G_{n}^{0}\right)}{2}
\end{aligned}
$$

where $n$ can take the values 1,2 , and $X$ and the index 0 denotes the corresponding data of the blank sample. These three sets of data are fitted independently using a standard model based on an appropriate approximation of the molecule detection function 76 . The fit parameters were equal for all three data sets. Generally, one finds three types of molecules in solution: A fraction $(g)$ that carries only the green label, a fraction that carries only the red label $(r)$, and a fraction of complexes which carry both labels $(m)$. From the fits of the correlation curves, the diffusion constant of the molecules, the amplitude $a_{n}^{c}$ of the diffusion term, and the uncorrelated intensity contribution $b_{n}^{c}$ for each curve are obtained. Here, $n$ stands for the focal volume 1,2 , or $X$ and $c$ for the observed color $G, R, M$. FCS theory predicts that the relative amplitude $\gamma_{n}=b_{n}^{G} / a_{n}^{G}$ is equal to the number of molecules within the focal volume carrying a green label

$$
\gamma_{n}=\frac{b_{n}^{G}}{a_{n}^{G}}=N_{n}^{g}+N_{n}^{m}
$$

where $N_{n}^{g}$ and $N_{n}^{m}$ are the average numbers of green and mixed-colored molecules in the $n$th detection volume, respectively. Similar relations hold for the red and mixed colors:

$$
\begin{aligned}
& \rho_{n}=\frac{b_{n}^{R}}{a_{n}^{R}}=N_{n}^{r}+N_{n}^{m} \\
& \mu_{n}=\frac{a_{n}^{M}}{b_{n}^{M}}=\frac{N_{n}^{m}}{\left(N_{n}^{g}+N_{n}^{m}\right)\left(N_{n}^{r}+N_{n}^{m}\right)}
\end{aligned}
$$


Typically, the focal volumes for the different colors do not have the same size. It is impossible to directly solve these equations for the different numbers of molecules. Instead, the focal volumes need to be calibrated. For this purpose, a diluted sample of doubly labeled dsDNA (25-25 bases, FCCS Standard Probe, IBA GmbH, Göttingen, Germany) was measured as a reference. Since this sample shows perfect codiffusion for all colors, one knows a priori that $\tilde{N}_{n}^{g}=\tilde{N}_{n}^{r}=0$ (all symbols with $\sim$ denote the reference sample). In case of identical volumes, one would expect to find $\tilde{\gamma}_{n}=\tilde{\rho}_{n}=1 / \tilde{\mu}_{n}$. For the actual, non-identical volumes, the calibration factors $c_{n}^{g}=1 / \tilde{\gamma}_{n} \tilde{\mu}_{n}$ and $c_{n}^{r}=1 / \tilde{\rho}_{n} \tilde{\mu}_{n}$ are introduced. For the overlap between the red and the green excitation/detection volume, the average number of molecules is then given by the simple relations

$$
\begin{aligned}
N_{n}^{g} & =c_{n}^{g} \gamma_{n}-N_{n}^{m} \\
N_{n}^{r} & =c_{n}^{r} \rho_{n}-N_{n}^{m} \\
N_{n}^{m} & =\mu_{n} c_{n}^{g} \gamma_{n} c_{n}^{r} \rho_{n}
\end{aligned}
$$

The relative numbers of molecules given by the cross-correlation amplitudes are calculated for each measurement. For the final data analysis, the mean value and the standard deviation of these two measurements are calculated.

The spectroscopic measurements were done by myself and I calculated all correlation functions, including the analysis and evaluation of the data. 


\subsection{Spliceosome purification, reconstitution, and labeling}

The entire sample preparation was carried out by Dr. Peter Odenwälder, Dr. Julia Dannenberg, and Dr. Thomas Ohrt from the group of Prof. Dr. Reinhard Lührmann at the Max Planck Institute for Biophysical Chemistry. A list of the used materials and a more detailed description of the biochemical methods are given in the $\mathrm{PhD}$ Thesis of Dr. Julia Dannenberg and Dr. Peter Odenwälder.

\subsubsection{The model system}

In this study, spliceosomal complexes isolated from the yeast Saccharomyces cerevisiae were used to investigate pre-mRNA splicing with FCCS. The yeast system is the most favored model organism. A lot of protocols are given and allow for genomic manipulation of the organism in an easy manner. It is possible to stall the spliceosomal complexes at defined stages because of given temperature-sensitive strains for different proteins. Functional spliceosomal complexes on a complete pre-mRNA strand can be purified and analyzed with various methods. The yeast proteins can be overexpressed and purified in E.coli in an easy manner. Because of these facts, the spliceosome can be assembled and reconstituted step by step and allows observations on different stages of the splice cycle.

Another important fact is the reduced number of involved proteins in a yeast spliceosome in comparison to the human spliceosome or other higher eukaryotes. Many splicing factors of the yeast spliceosome are evolutionarily conserved in human spliceosomes. The yeast spliceosome represents the core design of the splicing machinery 37 .

\subsubsection{Spliceosome purification}

Yeast spliceosomal complexes were purified essentially as described 37 except that heat inactivated extracts from the yeast strain prp2-1 [79] were used in the assembly reactions. Before splicing, Actin pre-mRNA wildtype or other constructs were incubated with a 35-fold molar excess of purified MS2-MBP fusion protein at $4^{\circ} \mathrm{C}$ for $30 \mathrm{~min}$ in $20 \mathrm{mM}$ HEPES-KOH (pH 7.9). Depending on the required amount of spliceosomal complexes, a 6 - $72 \mathrm{ml}$ splicing reaction containing $1.8 \mathrm{nM}$ of ${ }^{32} \mathrm{P}-$ labeled M3Act pre-mRNA (specific activity 20-300 cpm/fmol) was performed in $60 \mathrm{mM} \mathrm{K}-\mathrm{PO}_{4}$ (pH 7.4) (phosphate buffer was prepared by mixing solutions of 
$\mathrm{K}_{2} \mathrm{HPO}_{4}$ and $\mathrm{KH}_{2} \mathrm{PO}_{4}$ according to Sambrook et al. ${ }^{80}$ ), $3 \%$ PEG 8000, $2.5 \mathrm{mM}$ $\mathrm{MgCl}_{2}, 2.0 \mathrm{mM}$ ATP, $2.0 \mathrm{mM}$ spermidine, and $40 \%$ yeast extract in buffer D (see table below). $\mathrm{B}^{\text {act }}$ complexes were assembled by incubation at $23^{\circ} \mathrm{C}$ for $50 \mathrm{~min} .6 \mathrm{ml}$ aliquots of the splicing reaction were loaded onto 36-ml linear $10-30 \%$ (w/w) glycerol gradients containing GK150 or in some experiments GK75 buffer. Gradients were centrifuged for $14 \mathrm{~h}$ at 23,000 rpm in a SureSpin 630 rotor (Thermo Fisher Scientific, Pittsburgh, USA) and harvested manually in $1500 \mu$ fractions from the top. Fractions were analyzed by Cherenkov counting in a scintillation counter and peak fractions containing complexes were pooled and loaded onto columns containing 250-300 $\mu \mathrm{l}$ of amylose matrix equilibrated with GK75 or GK150 buffer. The matrix was washed twice with $10 \mathrm{ml}$ GK150 buffer and twice with GK75 buffer supplemented with $5 \%$ glycerol and $0.01 \%$ NP40, and spliceosomal complexes were eluted stepwise with $750 \mu$ lelution buffer. The elution fractions were either used directly for reconstitution assays or for FCCS analysis or were loaded onto a 3.8-ml linear $10-30 \%$ (w/w) glycerol gradient containing GK75 buffer. Gradients were centrifuged for $107 \mathrm{~min}$ at 490,000 g in a Sorvall TH660 rotor, and $175 \mu$ l fractions were harvested from the top of the gradient and analyzed by Cherenkov counting. Peak fractions were then used for mass spectrometry analysis or used in reconstitution assays.

Buffers for purification:

$\begin{array}{lll}\text { Buffer D } & 20 \mathrm{mM} & \text { HEPES-KOH pH } 7.9 \\ & 50 \mathrm{mM} & \text { KCl } \\ & 0.2 \mathrm{mM} & \text { EDTA pH } 8.0 \\ & 20 \%(\mathrm{v} / \mathrm{v}) & \text { glycerol } \\ & 0.5 \mathrm{mM} & \text { DTT } \\ & 0.5 \mathrm{mM} & \text { PMSF } \\ \text { GK150/GK75 buffer } & 20 \mathrm{mM} & \text { HEPES-KOH pH } 7.9 \\ & 1.5 \mathrm{mM} & \text { MgCl }_{2} \\ & 150 \mathrm{or} 75 \mathrm{mM} & \text { KCl }^{-K} \\ \text { Elution buffer with } & 12-20 \mathrm{mM} & \text { maltose } \\ \text { GK75 buffer } & 5 \% & \text { glycerol } \\ & 0.01 \% & \text { NP40 }\end{array}$




\subsubsection{Splicing reconstitution assays}

$10 \mathrm{fmol}$ of $\mathrm{B}^{\mathrm{act} \Delta \operatorname{Prp} 2}$ complexes eluted from the amylose matrix or from peak fraction(s) of the second gradient were supplemented with 100 fmol (10-fold molar excess) of recombinant proteins in a $40 \mu$ reaction.

After incubating the reconstitution reaction for $10 \mathrm{~min}$ on ice, $1 / 10$ volume of a 10-fold "rescue" solution was added to the reaction. The reactions were incubated for $45 \mathrm{~min}$ at $23^{\circ} \mathrm{C}$. After Proteinase $\mathrm{K}$ digestion, the RNAs were extracted by phenol-chloroform-isoamyl alcohol (PCI) extraction, precipitated by ethanol, and then analyzed by $8 \%$ denaturing PAGE followed by autoradiography.

For reconstitution assays on the amylose matrix, complex concentration was determined by Cherenkov counting and recombinant proteins were added in 10-fold molar excess. A typical reconstitution reaction was performed with 250-300 $\mu$ l amylose beads in a final volume of 500-600 $\mu \mathrm{l}$ in GK75 buffer containing 50-60 $\mu \mathrm{l}$ 10-fold "rescue" solution and were incubated at $23^{\circ} \mathrm{C}$ for $45 \mathrm{~min}$. For ATP depletion, the reaction was supplemented with $2 \mathrm{mM}$ glucose and 2 units of recombinant hexokinase and incubated for $10 \mathrm{~min}$ at $23^{\circ} \mathrm{C}$. The matrix was then washed thoroughly with 100 column volumes of GK75 buffer and the complexes were eluted with 12$20 \mathrm{mM}$ maltose. For dual-color FCCS measurements, reactions were carried out in solution in a final volume of $60 \mu \mathrm{l}$ with a final complex concentration of $1.0 \mathrm{nM}$. The purified, labeled $\mathrm{B}^{\text {act } \Delta \operatorname{Prp} 2}$ spliceosomes were then complemented with a 10 -fold molar excess of recombinant proteins and the extent of binding of Prp16 and Slu7 fused to EGFP or Cwc25-Alexa488 to the spliceosome was measured. Instead of adding the 10-fold rescue mix, the reaction conditions were only adjusted to final concentrations of $2.5 \mathrm{mM} \mathrm{MgCl}_{2}$ and $2 \mathrm{mM}$ ATP (or ATP analogues), as PEG8000 increased the background of the FCCS measurements.

10x rescue solution $200 \mathrm{mM}$ phosphate buffer $\mathrm{K}-\mathrm{PO}_{4}(\mathrm{pH} 7.3)$

$10 \mathrm{mM} \quad \mathrm{MgCl}_{2}$

$20 \mathrm{mM} \quad$ ATP

$14 \% \quad$ PEG 8000 


$\begin{array}{lll}\text { GK75 buffer } & 20 \mathrm{mM} & \text { HEPES-KOH pH } 7.9 \\ & 1.5 \mathrm{mM} & \mathrm{MgCl}_{2} \\ 75 \mathrm{mM} & \mathrm{KCl} \\ 5 \% & \text { glycerol } \\ & 0.01 \% & \text { NP40 } \\ & 0.5 \mathrm{mM} & \text { DTT } \\ 0.5 \mathrm{mM} & \text { PMSF }\end{array}$

\subsubsection{Denaturing polyacrylamide gel electrophoresis}

Gel electrophoresis is a method for separation of macromolecules, i.e. DNA, RNA, and proteins, based on their size and charge. By applying an electric field, negatively charged molecules move through a polyacrylamide gel. This gel is a crosslinked polymer with a mesh network. Smaller and thereby faster molecules migrate more easily through the pores of the gel than larger molecules. The movement is also influenced by the charge density of the molecule. By using a denaturing gel, the natural structure of the molecule is disrupted and it is unfolded into a linear chain. The mobility of the molecule through the gel then depends on its linear length and its mass-to-charge ratio 34 . Using denaturing polyacrylamide gel electrophoresis allows for separating the splicing products, i.e. the RNAs up to $1500 \mathrm{bp}$ in size. For the denaturing of RNA, the gel contains $8 \mathrm{M}$ urea and acrylamide concentrations between $5 \%$ and $10 \%$. The acrylamide polymerization was initiated by adding ammonium persulfate and TEMED. The RNA samples were resuspended in RNA loading dye, denatured at $70^{\circ} \mathrm{C}$ for $3 \mathrm{~min}$, and loaded on the gel. For electrophoresis $1 \mathrm{x}$ TBE buffer (10x TBE buffer: 0.89 M Tris, 0.89 M boric acid, 25 mM EDTA pH 8.0) and a constant voltage were used. For a better separation of the splicing products and intermediates, an optimized ratio of acrylamide to bisacrylamide of 29:1 was used. The RNAs were visualized by autoradiography, which is a photographic technique for detection of radioactive material, i.e. radioactive labeled nucleotides.

\subsubsection{Labeling of spliceosomes}

Proteins were genetically modified by fusion to enhanced green fluorescent protein (eGFP). The different $\mathrm{B}^{\text {act } \Delta \mathrm{Prp} 2}$ complexes were assembled in vitro using heatinactivated splicing extracts from yeast strains that were temperature-sensitive in the ATPase Prp2 [79] and which stably expressed the protein of interest as an eGFP 
fusion protein. The purified, doubly labeled $\mathrm{B}^{\text {act } \Delta \operatorname{Prp} 2}$ spliceosomes were then complemented with recombinant Prp2, Spp2, and ATP. Recombinant Spp2 was always added together with Prp2, as our purified $\mathrm{B}^{\text {act } \Delta \operatorname{Prp} 2}$ complexes lack Spp2 48. Recombinant Cwc25 was chemically labeled with Alexa488 as described below.

\section{Labeling of pre-mRNAs with a fluorescent dye}

Guanosine-5'-O-monophosphorothioate (5'-GMPS) can be incorporated as the first nucleotide by T7 RNA polymerase in vitro. 5'-GMPS-containing mature actin premRNA was prepared by in vitro transcription of Acc65I digested pUC18-Actin-wt plasmid DNA with T7 RNA polymerase (40 mM Tris- $\mathrm{HCl}$, pH 8.0, $30 \mathrm{mM} \mathrm{MgCl}_{2}$, $10 \mathrm{mM}$ DTT, $2 \mathrm{mM}$ spermidine, $0.1 \mathrm{U}$ yeast inorganic pyrophosphatase (NEB), 0.5 U RNasin (Promega), 4 mM ATP, 4 mM CTP, 4 mM UTP, 1 mM GTP, $10 \mathrm{mM}$ 5'GMPS (Biolog); $37^{\circ} \mathrm{C}, 3 \mathrm{~h}$ ). Transcripts were purified by electrophoresis through $5 \%$ polyacrylamide, $8 \mathrm{M}$ urea gels and passively eluted into $0.5 \mathrm{M} \mathrm{NaOAc}$, pH 5.3, 1 mM EDTA, pH 8.0, $2.5 \%$ phenol-chloroform-isoamyl alcohol. To link the 5'GMPS-modified pre-mRNA covalently to the fluorescent dye Atto647N (ATTOTEC), Atto647N-maleimide was incubated with the pre-mRNA in the presence of $100 \mathrm{mM} \mathrm{NaPO}_{4}$ (pH 7.2) at RT for $4 \mathrm{~h}$. The Atto647N-labeled pre-mRNA was prepurified by $4 \mathrm{x}$ ethanol precipitation and several washing steps to remove unreacted dye, resulting in nearly $100 \%$ labeled pre-mRNA. The dye:RNA stoichiometry was calculated according to the manufacturer's protocol (Molecular Probes).

\section{Expression and Purification of Prp16-EGFP and Slu7-EGFP}

Full-length yeast PRP16 and $S L U^{\prime 7}$ genes were cloned into pYES2/CT plasmid containing an N-terminal $\mathrm{His}_{6}$-tag in frame with an N-terminal eGFP-tag and transformed into the INVSc1 yeast strain (Invitrogen). Cells were grown to an $\mathrm{OD}_{600}$ of 4.0 in YP medium supplemented with $2 \%$ galactose. Whole cell extract was prepared as described $\stackrel{\text { 81. }}{2}$ His $_{6}$-eGFP-Prp16 and His ${ }_{6}$-eGFPSlu7 were captured on a $\mathrm{Ni}^{2+}$-NTA resin (GE Healthcare), eluted with the GK75 buffer containing $200 \mathrm{mM}$ imidazole, and purified by size exclusion chromatography (S200 Sephadex 16/600 (GE Healthcare)) equilibrated with $20 \mathrm{mM}$ HEPES pH 7.5, $200 \mathrm{mM} \mathrm{NaCl}, 20 \%$ (w/v) glycerol, and $2 \mathrm{mM}$ DTT. Pooled fractions were dialyzed against $40 \mathrm{mM}$ Tris$\mathrm{HCl}, \mathrm{pH}$ 7.5, $150 \mathrm{mM} \mathrm{NaCl}, 20 \%$ (w/v) glycerol, and $1 \mathrm{mM}$ DTT. Recombinant Prp2, Spp2, Cwc25, Prp16, Slu7, Prp18 and Prp22 were expressed in E.coli and purified as described previously 48 . 


\section{Labeling of Cwc25}

Cwc25 does not contain any cysteine in its amino acid sequence. For fluorescent labeling a single cysteine at the C-terminus was added. The DNA sequence of the construct was confirmed by sequence analysis. The cysteine mutant, Cwc25-C was labeled with the fluorescent reagent Alexa488-maleimide (Invitrogen). The labeling was performed in $100 \mathrm{mM} \mathrm{NaPO}$-buffer ( $\mathrm{pH}$ 7.2) which contained $10 \mu \mathrm{M}$ reducing agent tris(2-carboxyethyl)phosphine (TCEP) (Sigma). The reaction mixture contained $20 \mathrm{nM}$ protein and $2 \mathrm{mM}$ Alexa488-maleimide, which was added in DMF. The reaction was allowed to proceed at $20^{\circ} \mathrm{C}$ for $2 \mathrm{~h}$. The reaction was stopped by adding GSH. Excess of the reagents was removed by gel filtration on a PD10 gel filtration column equilibrated with GK75. The degree of labeling was calculated according to the manufacturer protocol (Jena Bioscience) and yielded $73 \%$ of labeled Cwc25 at the C-terminus.

\subsection{Measurement protocol}

Spliceosome complexes were assembled on pre-mRNAs carrying the fluorescent label Atto647 at its 5'-end. The complexes were purified as described above and supplemented with recombinant proteins on a amylose matrix ${ }^{54}$. All samples were kept on ice until the measurements. For the FCCS measurements, the complexes were measured in a volume of $25 \mu \mathrm{l}$ at a concentration of $1.0 \mathrm{nM}$ in a measurement chamber. The chamber consisted of two glass coverslides connected through a $1 \mathrm{~mm}$ silicon spacer (Grace Bio-Labs). To avoid unspecific binding to the coverslides during the measurements, the coverslides were coated with Sigmacote (Sigma-Aldrich) according to the manufacturer's instructions before the experiments. Two independent measurements over $10 \mathrm{~min}$ of each sample were performed at room temperature. 


\section{Results}

In previous studies, changes in the protein composition and dynamics of the spliceosome were observed with biochemical methods, which provided initial indications of the protein content and dynamics. Only little is known about the order of recruitment, release, and degree of binding of the different spliceosomal proteins during maturation and catalysis of the spliceosome. The ordered recruitment and release of proteins and RNA is important for the spliceosome to become a functional multiprotein complex. To gain new insights into the dynamic behavior of spliceosomal proteins in solution at low concentrations, FCCS is a powerful and ideal technique. The advantage of using FCCS in solution is that the spliceosomal maturation can be investigated in thermal equilibrium and in solution without the need of additional purification steps.

Cwc22 was used to study the temporal stability of the spliceosome during FCCS experiments because it is a splicing factor and not a core component of the spliceosome as shown by mass spectrometry ${ }^{37}$. To study the feasibility of FCCS for the dynamic analysis of the spliceosome, the behavior of Snu114 tagged with eGFP was investigated as this protein represents a stable component during spliceosomal activation and catalysis 3782 . Then, binding behavior and interactions of several proteins (Cwc25, Prp16, Slu7/Prp18) at particular steps of the splice cycle were studied.

\subsection{Preliminary experiments for testing the availability of FCCS on the spliceosomal complex}

To test FCCS for monitoring the binding behavior of different spliceosomal proteins, measurements on the $\mathrm{B}^{\text {act } \Delta \operatorname{Prp} 2}, \mathrm{~B}^{*}, \mathrm{C}, \mathrm{C}^{*}$, and $\mathrm{C} 2 \mathrm{nd}$ complex were performed. The $\mathrm{B}^{\text {act } \Delta \operatorname{Prp} 2}$ is the spliceosomal complex stalled before Prp2 action at the stage of the $\mathrm{B}^{\text {act }}$ complex by using a temperature-sensitive Prp2 mutant 48 (see fig. 19). The B* complex is the $\mathrm{B}^{\text {act } \Delta \operatorname{Prp} 2}$ complex complemented with Prp2, Spp2, and ATP. Further addition of Cwc25 leads to the $\mathrm{C}$ complex. Complementation with Prp16 results in the formation of the $\mathrm{C}^{*}$ complex and further addition with Slu7/Prp18 leads to the C2nd complex. In all measurements, the pre-mRNA was fluorescently labeled with the red dye Atto647N and the protein of interest was labeled with eGFP. 


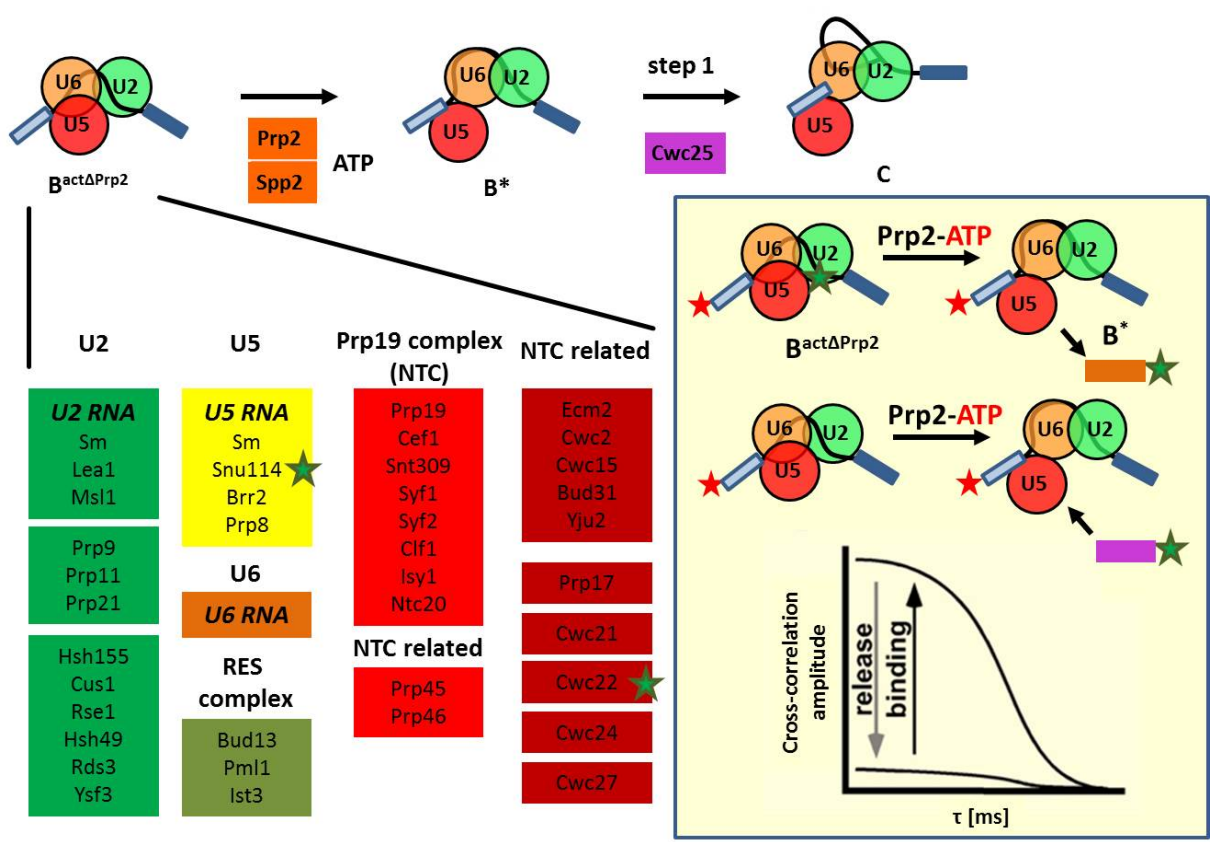

Figure 19: Schematic representation of the experimental design and data. The protein composition of the stalled, Prp2-deficient $B^{\text {act } \Delta \text { Prp2 }}$ complex is shown. The green stars indicate the proteins labeled with eGFP. Box on the right site: representation of the changes in the cross-correlation amplitude induced by release or binding of proteins.

(Figure adapted from Prior et al. ${ }^{[54]}$ )

In the FCCS measurements, the sample has to fulfill the following criteria. First, the sample has to be stable over $3 \mathrm{~h}$ at a concentration of $\sim 1 \mathrm{nM}$ which would be an indication of the stable integrity of the complex. Second, the complexes as well as the supplied proteins should not aggregate during the measurements.

To check the sample stability over time, FCCS measurements on Cwc22 and the B ${ }^{\text {act }}$ complex were performed and repeated after $3 \mathrm{~h}$. Cwc22 was chosen because it gives a good measure on spliceosomal integrity as it is not a core component but stably bound during spliceosomal maturation 83 . The results are shown in figure 20. The measurements showed that the criteria (no aggregation over 3 hours at $\sim 1 \mathrm{nM}$ concentration) are fulfilled indicated by comparable high cross-correlation amplitudes around $35 \%$. The long lifetime and stability of the complexes was observed by FCCS.

The correlation curves themselves could indicate the influence of aggregation in FCCS experiment and the curves for Cwc22's binding to the $\mathrm{B}^{\text {act }}$ complex at time point zero and after $180 \mathrm{~min}$ are shown in figure 21. The correlation curves were well-defined and could be fitted perfectly even after 3 hours. This showed that aggregation of complexes did not occur. 


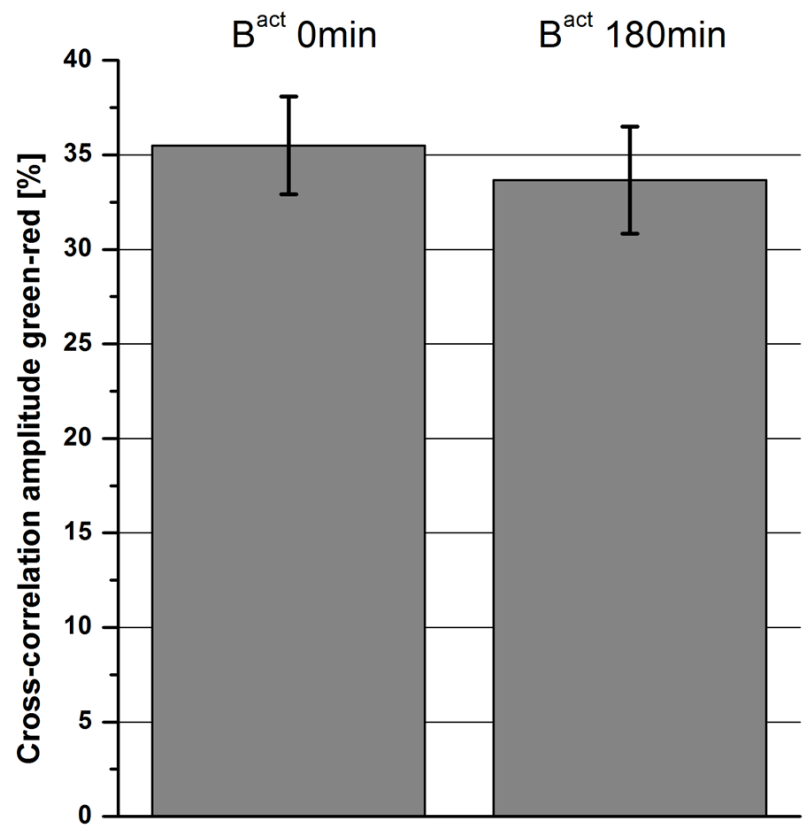

Figure 20: Binding behavior of Cwc22-eGFP to the $B^{\text {act }}$ complex at time point zero and after $180 \mathrm{~min}$. For each data point, the mean and the standard deviation of two independent measurements were calculated.

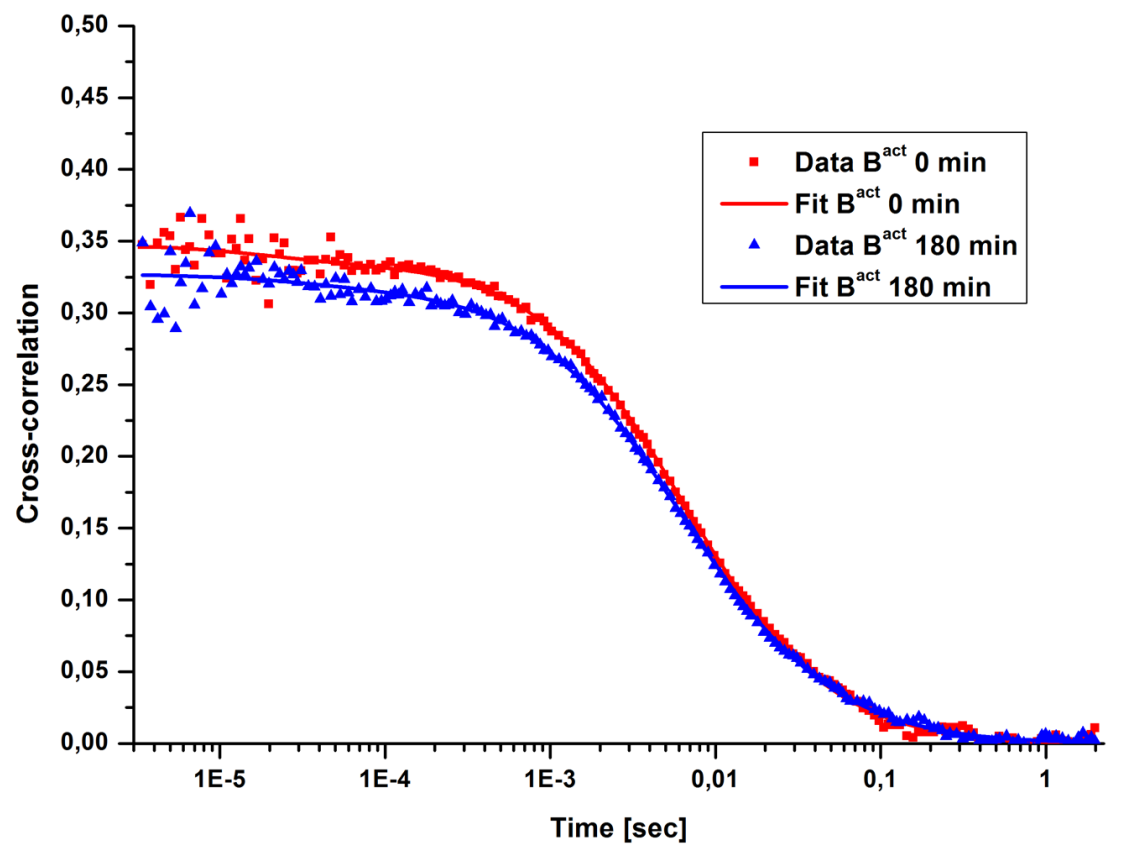

Figure 21: Normalized data points and fits of the cross-correlation curves for Cwc22's binding to the $B^{\text {act }}$ complex at time point zero and after $180 \mathrm{~min}$. Exemplary curves of one measurement at time point zero and of one measurement after $3 \mathrm{~h}$ are shown. 
The following control experiments were performed to ensure that the purified spliceosomes behave in the same way in all FCCS experiments and that the addition of further proteins has no disturbing effect. The well-characterized protein Snu114 was chosen as this protein remains bound to the spliceosome and pre-mRNA from the B complex over the complete splice cycle 82 .

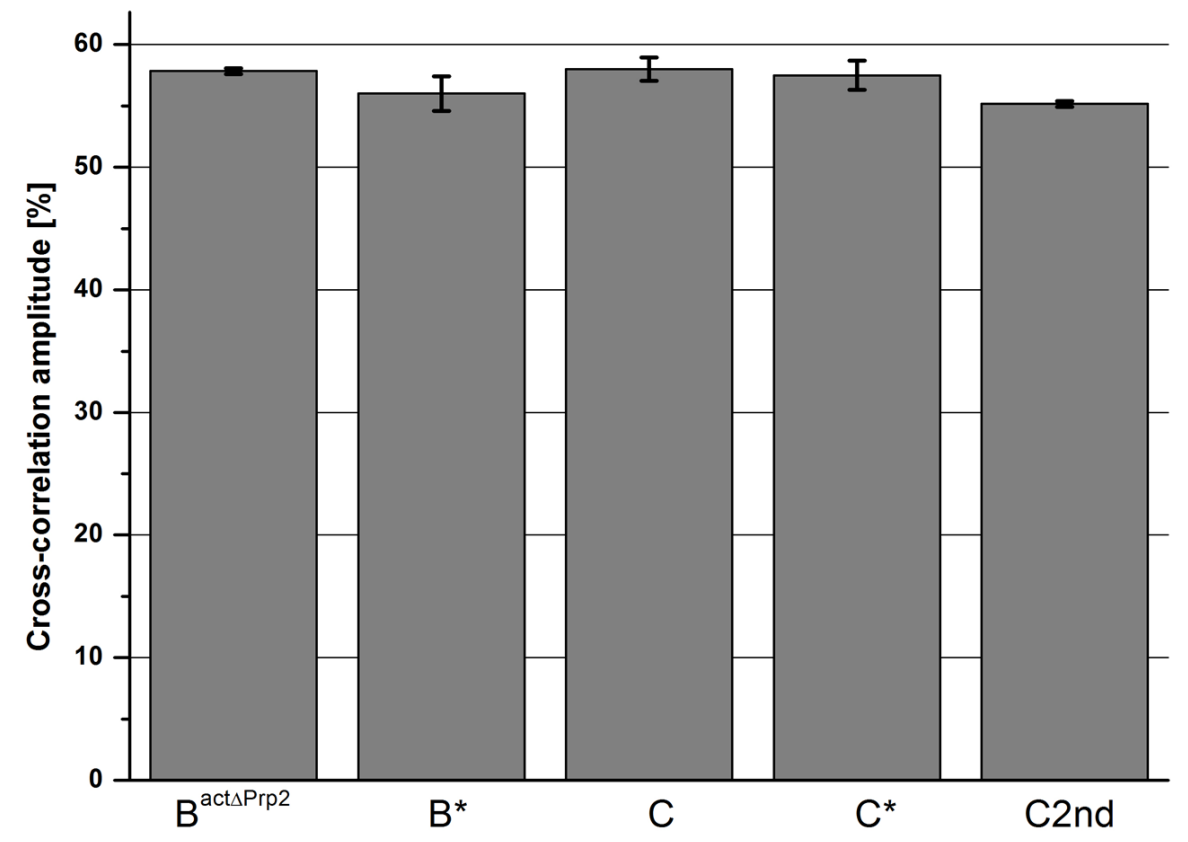

Figure 22: Binding behavior of Snu114-eGFP. Cross-correlation amplitudes for the binding behavior of Snu114-eGFP to the spliceosomal complexes $B^{\text {act } \Delta P r p 2}, B^{*}, C, C^{*}$, and $C 2 n d$. For each data point, the mean and the standard deviation of two independent measurements were calculated. (Parts of this figure also appeared in the PhD thesis of Julia Dannenberg and are published in Prior et al. ${ }^{[54}$ )

The results of the measurements on Snu114-eGFP are shown in figure 22. The crosscorrelation amplitude was at a high level of $\sim 57 \%$ for the $\mathrm{B}^{\text {act } \Delta \mathrm{Prp} 2}$ complexes which contain Snu114-eGFP. This showed that the $\mathrm{B}^{\text {act } \Delta \operatorname{Prp} 2}$ complex did not dissociate at the conditions of the FCCS measurement.

By incubating Snu114-eGFP-B ${ }^{\text {act } \Delta \operatorname{Prp} 2}$ with recombinant Prp2/Spp2 proteins plus $2.0 \mathrm{mM}$ ATP for $45 \mathrm{~min}$ at $23^{\circ} \mathrm{C}$, the catalytic activation of the complex was induced and the $\mathrm{B}^{*}$ complex was generated. As shown in figure 22 , the cross-correlation amplitude for the $\mathrm{B}^{*}$ complex was comparable to the one for the $\mathrm{B}^{\text {act } \Delta \operatorname{Prp} 2}$ complex. The relative number of Snu114-eGFP molecules bound to the complexes was constant at a high level even upon the activation of the spliceosome.

When the spliceosome underwent the first catalytic step, induced by the addition 
of Cwc25, Snu114-eGFP was still bound which was shown by a constantly crosscorrelation amplitude of over $55 \%$. The further addition of Prp16 and Slu7/Prp18 did not change the level of the cross-correlation amplitude and Snu114-eGFP was stable bound when the spliceosome underwent the second catalytic step. These measurements showed that the addition of proteins, for inducing the activation and catalytic steps of the spliceosome, did not generate aggregations of complexes or further artefacts. These measurements were performed at low salt concentration of $75 \mathrm{mM} \mathrm{KCl}$.

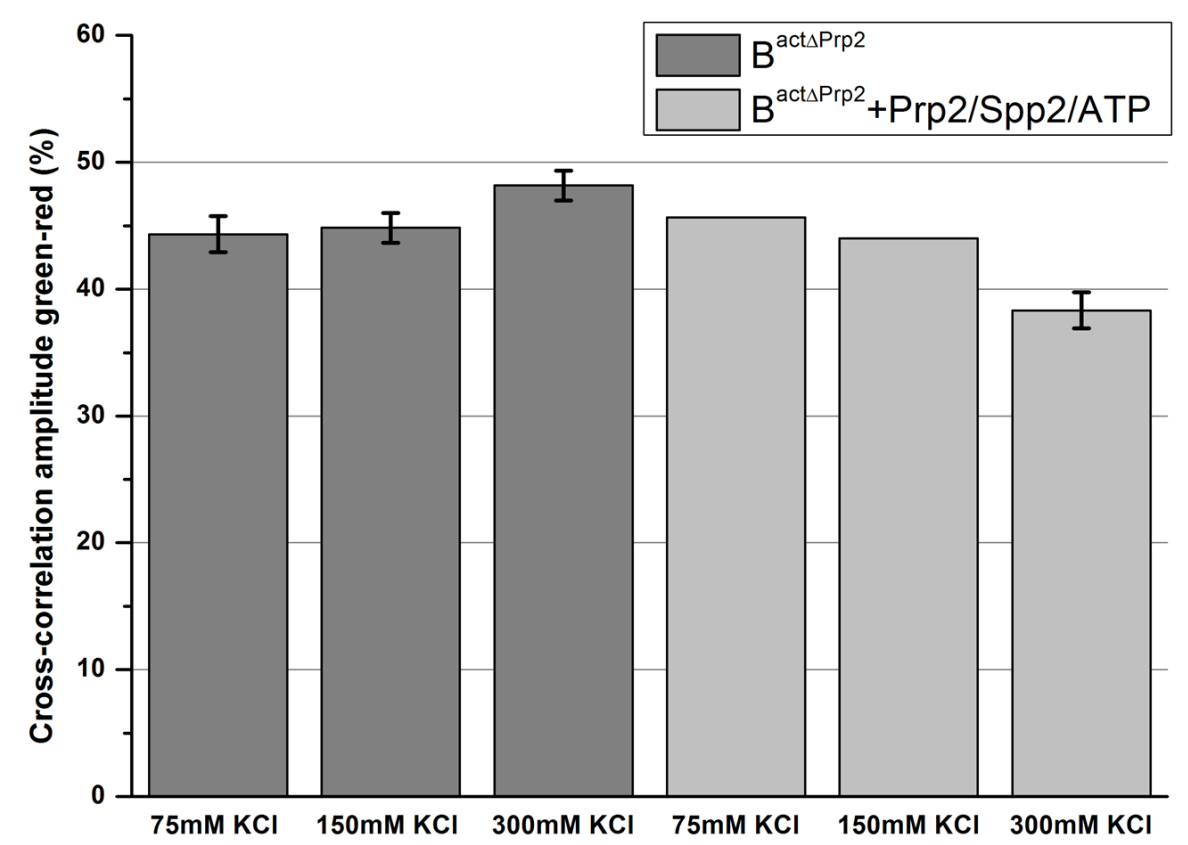

Figure 23: Binding behavior of Snu114-eGFP at different salt concentrations. Binding behavior of Snu114-eGFP to the spliceosomal $B^{\text {act } \triangle P r p 2}$ and $B^{*}$ complex at different salt concentrations ( $75 \mathrm{mM} \mathrm{KCl}, 150 \mathrm{mM} \mathrm{KCl}, 300 \mathrm{mM} \mathrm{KCl}$ ). (Figure also appeared in the PhD thesis of Julia Dannenberg and published in Prior et al. [54])

In the next experiment, the concentration of $\mathrm{KCl}$ was varied $(75 \mathrm{mM}, 150 \mathrm{mM}$, $300 \mathrm{mM}$ ) and the cross-correlation amplitude was measured for the $\mathrm{B}^{\text {act } \Delta \operatorname{Prp} 2}$ and $\mathrm{B}^{*}$ complex. The higher salt concentration subjects the spliceosome to harsher conditions, which allowed for testing the binding stability of Snu114 to both complexes. The results are shown in figure 23 . For both complexes, the cross-correlation amplitude was over $40 \%$ at different salt concentrations. Even harsh conditions like $300 \mathrm{mM} \mathrm{KCl}$ did not change the binding affinity of Snu114-eGFP to the $\mathrm{B}^{\text {act } \Delta \text { Prp2 }}$ complex. During the activation of the spliceosome by Prp2/Spp2 and at $\mathrm{KCl}$ concentrations of $150 \mathrm{mM}$ or $300 \mathrm{mM}$, the cross-correlation amplitude remained at a 
high level of over $35 \%$, which indicates that Snu114-eGFP was still bound. These results clearly show that Snu114 has a high-affinity binding site in the $\mathrm{B}^{\text {act } \Delta \operatorname{Prp} 2}$ and $\mathrm{B}^{*}$ complex and is in agreement with published results ${ }^{82 .}$. Additionally, the complexes are stable even at a concentrations of $\sim 1 \mathrm{nM}$.

Surprisingly, measurements at higher salt concentrations, i.e. $500 \mathrm{mM} \mathrm{KCl}$, were not possible because the spliceosomal complexes most likely aggregate and attach to the glass surface of the used coverslides.

To conclude, FCCS is a powerful method for monitoring the binding of proteins in a macromolecular complex at nanomolar concentrations from low to higher salt concentrations. The measurements showed that FCCS is an ideal tool for observing the dynamics of complex systems in solution and at equilibrium, and the method provides a fast readout of results. An advantage of FCCS is the small sample volume, which corresponds to small amounts of raw material. 


\subsection{Recruitment and release of Cwc25-Alexa488 studied by FCCS}

The requirements for Cwc25's recruitment to and dissociation from the spliceosome were investigated with FCCS. Measurements on spliceosomal complexes which undergo the catalytic steps were performed. Different pre-mRNA substrates were used to monitor the influence of the substrate type on the binding of Cwc25.

\subsubsection{Binding of Cwc25-Alexa488 to the actin wildtype pre- mRNA}

In the first step, the binding behavior of Cwc25 to complexes assembled on actin wildtype (Actwt) pre-mRNA was observed. For the experiments, purified $\mathrm{B}^{\text {act }}$ complexes assembled on Actwt pre-mRNA were bound to an amylose affinity matrix and incubated with Prp2, Spp2, ATP, and Cwc25-Alexa488. By incubation with hexokinase and glucose, ATP was removed. Afterwards, the column was washed with GK75 buffer. The produced C complexes were eluted from the affinity matrix with maltose. Additionally, Prp16 and Slu7/Prp18 were added to the solution. This was done with and without ATP, or with non-hydrolysable ATP analog AMP-PNP or AMP-PCP. The solutions were incubated for $45 \mathrm{~min}$ at $23{ }^{\circ} \mathrm{C}$ and used for FCCS measurements. In all measurements, the pre-mRNA was labeled with the red dye Atto647N and Cwc25 was labeled with Alexa488.

The results of the FCCS measurements are shown in figure 24. The cross-correlation amplitude for the $\mathrm{B}^{\text {act }}$ complex supplemented with Cwc25-Alexa488 was 1 \% (column $\mathrm{B}^{\text {act }}$ in figure 24). This shows that Cwc25 cannot bind to the $\mathrm{B}^{\text {act }}$ complex. The low cross-correlation amplitude corresponds to the background which appears in all FCCS measurements of the spliceosome. By the addition of Prp2, Spp2, and ATP, the C complex was formed. The cross-correlation amplitude increased from $\sim 1 \%$ to $\sim 31 \%$ which indicates that Cwc25 was recruited to the spliceosome during $\mathrm{B}^{*}$ complex formation and was stably bound to the $\mathrm{C}$ complex. Further incubation with Prp16 and ATP led to the formation of the $\mathrm{C}^{*}$ complex but not to the release of Cwc25 from the complex. The cross-correlation amplitude for the $\mathrm{C}^{*}$ complex was slightly reduced to $26 \%$ but was still comparable to the one for the C complex. The cross-correlation amplitude was also comparable to the one obtained from the reaction of the $\mathrm{C}$ complex with Prp16 and AMP-PNP (column $\mathrm{C}^{*}+\mathrm{AMP}-\mathrm{PNP}$ ). These results showed that Prp16 and its ATP-dependent catalytic activation for 


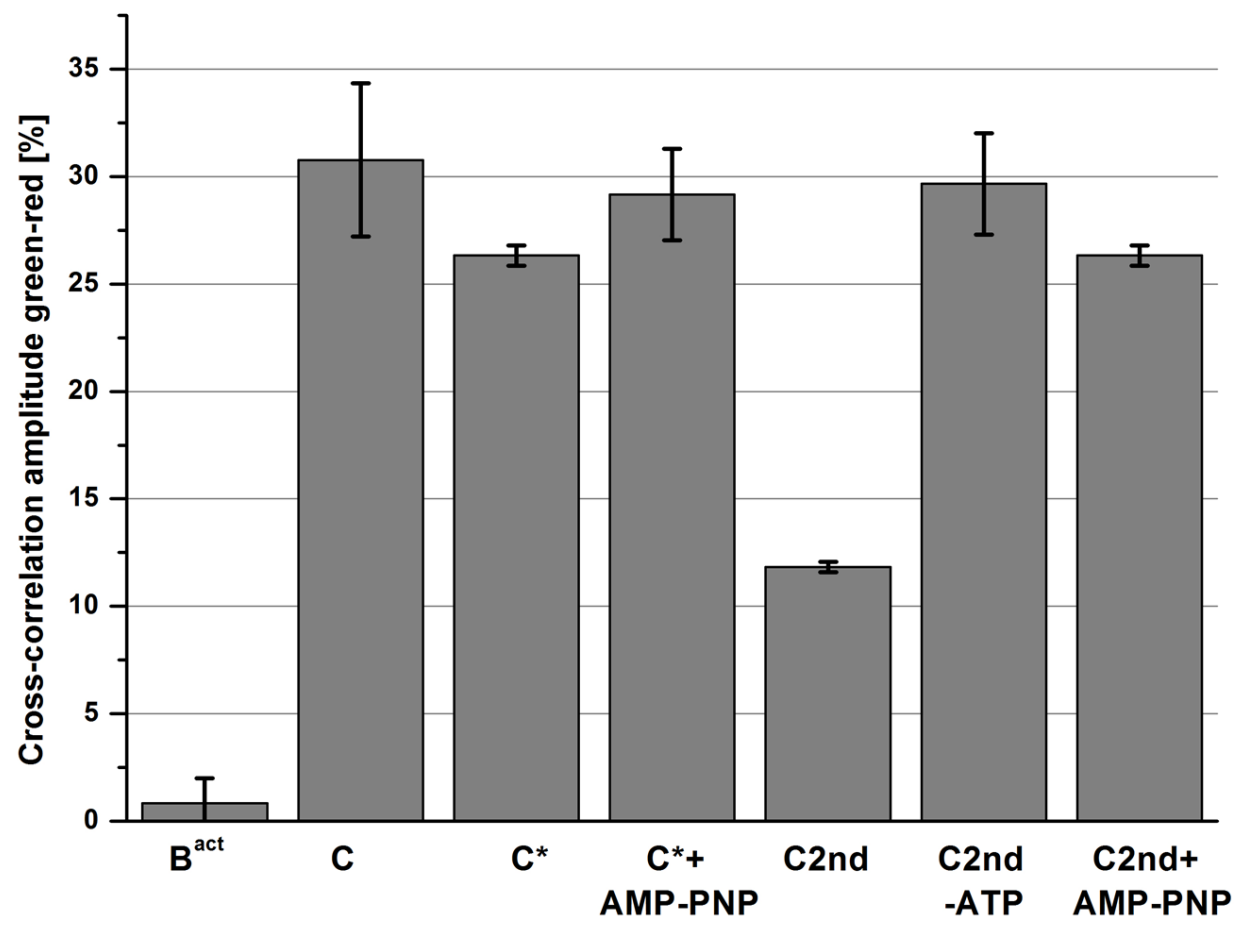

Figure 24: Binding of Cwc25-Alexa488 to Actwt pre-mRNA. Eluted C complexes were complemented in solution with Prp16 and Slu7/Prp18. FCCS measurements were performed upon the addition of ATP, AMP-PNP or without ATP. (Figure also appeared in the PhD thesis of Peter Odenwälder and Ohrt et al. ${ }^{84}$ )

the step 2 catalysis are not sufficient for the release of Cwc25. Further addition of Slu7/Prp18 to the $\mathrm{C}^{*}$ complexes led to a low cross-correlation amplitude of $\sim 12 \%$. This indicates that Cwc25 dissociated from the complex. A comparable release was not observed for C complex incubated with Prp16 and Slu7/Prp18 without ATP or with AMP-PNP.

In conclusion, these results showed that for the release of Cwc25, the ATPase activity of Prp16 and Slu7/Prp18 are necessary. The addition of only Prp16 is not sufficient for the dissociation of Cwc25 from the spliceosome.

\subsubsection{Binding affinity of Cwc25 to the $\mathrm{B}^{*}$ complex}

Initially, the binding of Cwc25 to the $\mathrm{B}^{*}$ complex was studied in detail. The binding affinity of Cwc25 to the spliceosome changed drastically from the $\mathrm{B}^{\text {act }}$ to the $\mathrm{B}^{*}$ complex (see fig. 25). Therefore, the binding constant for Cwc25 to the $\mathrm{B}^{\text {act }}$ and $\mathrm{B}^{*}$ complexes was measured. By choosing these two complexes, the binding affinity of Cwc25 immediately before and after catalytic activation by Prp2 could be 


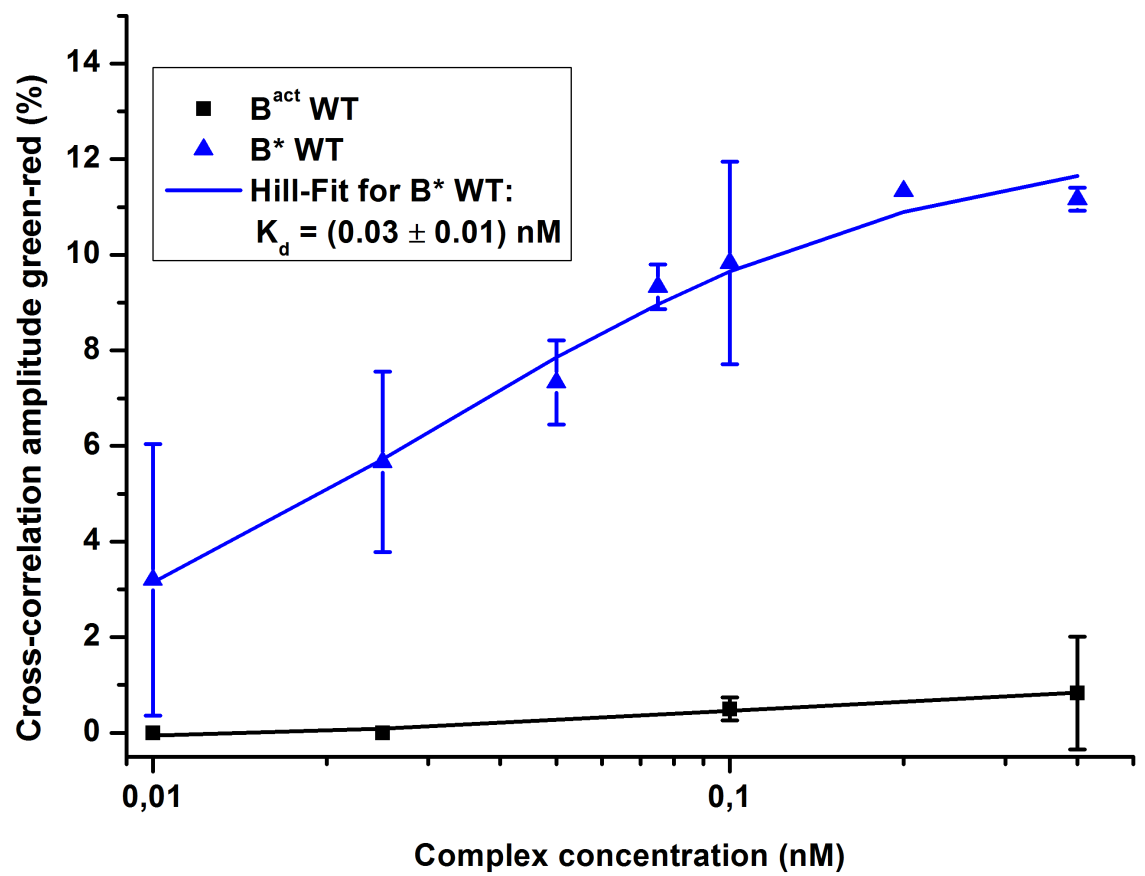

Figure 25: Titration of $B^{\text {act } \Delta P r p 2}$ complexes and $B^{*}$ complexes to Cwc25Alexa488. Cross-correlation amplitudes for the titration of $B^{\text {act } \Delta \operatorname{Prp} 2}$ and $B^{*}$ complexes to $0.3 \mathrm{nM}$ Cwc25-Alexa488. The data points were fitted by a Hill plot and yielded a dissociation constant of Cwc25-Alexa488. (Figure also appeared in the PhD thesis of Peter Odenwälder and published in Prior et al. [54])

investigated. To measure the binding curves, increasing amounts of the complexes were added to a fixed concentration of Cwc25-Alexa488 of $0.3 \mathrm{nM}$.

The changes in the cross-correlation amplitude with complex concentration are shown in figure 25. It was not possible to observe a binding curve of Cwc25 to the $\mathrm{B}^{\text {act }}$ complex because Cwc25 was not bound to this complex. However, a binding curve was observed for the $\mathrm{B}^{*}$ complex (see fig. 25). Data points were fitted using the Hill equation

$$
y=\frac{x^{n}}{K_{d}^{n}+x^{n}}+C
$$

where $y$ is the cross-correlation amplitude, $x$ the complex concentration, $n$ the Hill coefficient, $K_{d}$ the binding constant, and $C$ a constant taking into account some offset in the binding curve. For the fitting, $n=1$ was assumed to describe a noncooperative one-to-one binding behavior. The mean $K_{d}$ and its error were estimated by a bootstrapping method taking into account the standard deviation of each data 
point $\frac{85}{85}$. The fit of the Hill equation to the data points of the $\mathrm{B}^{*}$ complex yielded a binding constant of $K_{d}=(0.03 \pm 0.01) \mathrm{nM}$. This low binding constant indicates a high binding affinity of Cwc25 to the complex after the action of Prp2.

These measurements showed on the one hand, that FCCS can be used to monitor very low binding constants between two interaction partners which have large differences in size, i.e. the spliceosome and one small protein. On the other hand, that the Prp2-dependent rearrangements generated a high-affinity binding site for Cwc25. To obtain more insights into the mechanisms of Cwc25's release, further experiments on mutated pre-mRNAs were necessary. These are described in the following sections.

\subsubsection{Binding of Cwc25-Alexa488 to the ActACAC pre-mRNA}

Measurements on spliceosomes assembled on actin pre-mRNA with a mutated 3'SS were performed, to determine if the dissociation of Cwc 25 necessitates the second catalytic step. The used actin pre-mRNA carried an ACAC sequence instead of an AGAG one on the 3'SS (ActACAC pre-mRNA). Due to this mutation, the spliceosome cannot undergo the second catalytic step.

The results of FCCS measurements on spliceosomes assembled on ActACAC premRNA are shown in figure 26. Comparable to the results for the measurements on spliceosomes assembled on Actwt pre-mRNA, the cross-correlation amplitude was higher for the $\mathrm{C}$ (fig. 26, column C, 22\%) than for the $\mathrm{B}^{\text {act }}$ complex ( 1 \%). Cwc25 was bound to the $\mathrm{C}$ complex after the action of Prp2/Spp2. Whereas addition of Prp16 led to a small decrease in the cross-correlation amplitude to $20 \%$, the overall amplitude remained high suggesting that Cwc25 was still bound. Further addition of Slu7/Prp18 and ATP led to the release of Cwc25, shown by a reduced cross-correlation amplitude of $\sim 4 \%$. Measurements on $\mathrm{C}$ complexes incubated with Prp16 and Slu7/Prp18 with AMP-PCP or without ATP showed no significant change in the cross-correlation amplitude which remained between $18 \%$ and $19 \%$ in all cases. A release of Cwc25 was only observed for complexes incubated with Prp16, Slu7/Prp18, and ATP (fig. 26, column C2nd).

These data showed that Cwc25 is released from the spliceosome if the second catalytic step is blocked and Slu7/Prp18 is required for the dissociation of Cwc25. As a control experiment, the products of the splicing reaction were analyzed by denaturing gel electrophoresis (these experiments were performed by the lab of Prof. Lührmann) and aliquots from the same reactions that had been used for the 


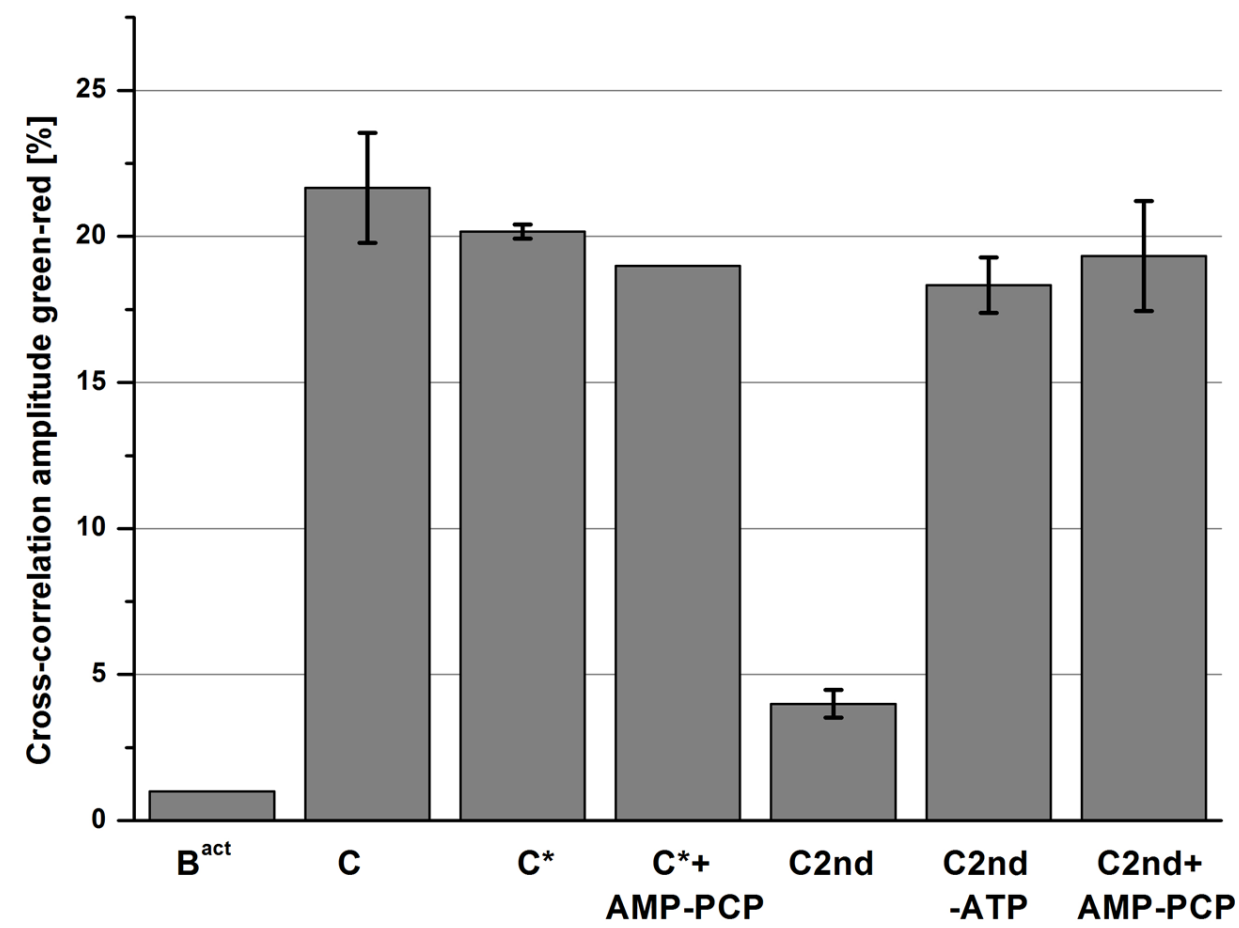

Figure 26: Binding of Cwc25-Alexa488 to ActACAC 3'SS mutated pre-mRNA. Eluted $C$ complexes were complemented in solution with Prp16 and Slu7/Prp18. FCCS measurements were performed upon the addition of ATP, AMP-PCP, or without ATP.

(Figure also appeared in the PhD thesis of Peter Odenwälder and in Ohrt et al. [84])

FCCS measurements were taken. The samples were loaded on a gel and visualized by autoradiography. The splicing products, i.e. the 5'exon, the intron-3'exon, the intron, and both exons, are visualized by bands. The electrophoresis experiment showed that the second catalytic step did not occur because splicing products were not detected (see fig. 27, left site). In contrast, a comparable gel of the spliceosomes assembled on Actwt pre-mRNA showed that the first as well as the second catalytic step proceed which can be concluded from the presence of bands corresponding to the splicing products (see fig. 27, right site).

The measurements on Act pre-mRNA and ActACAC pre-mRNA showed that Slu7/Prp18 is required for the displacement of Cwc25 from the spliceosome irrespective of whether or not the second step occurs. The used pre-mRNA has a long distance between the branch site and the 3'SS of 38 nucleotides. For this reason, Slu7/Prp18 is essential to bind the 3'SS efficiently into the step 2 active site ${ }^{61}$. It was shown by crosslinking experiments that Slu7 directly contacts mutant 3'SS 61] and may also facilitate docking of the 3'SS ACAC mutant. It is possible that the actual docking of the 3'SS to the step 2 active site induces the release of Cwc25 from 


\section{ActACAC pre-mRNA}

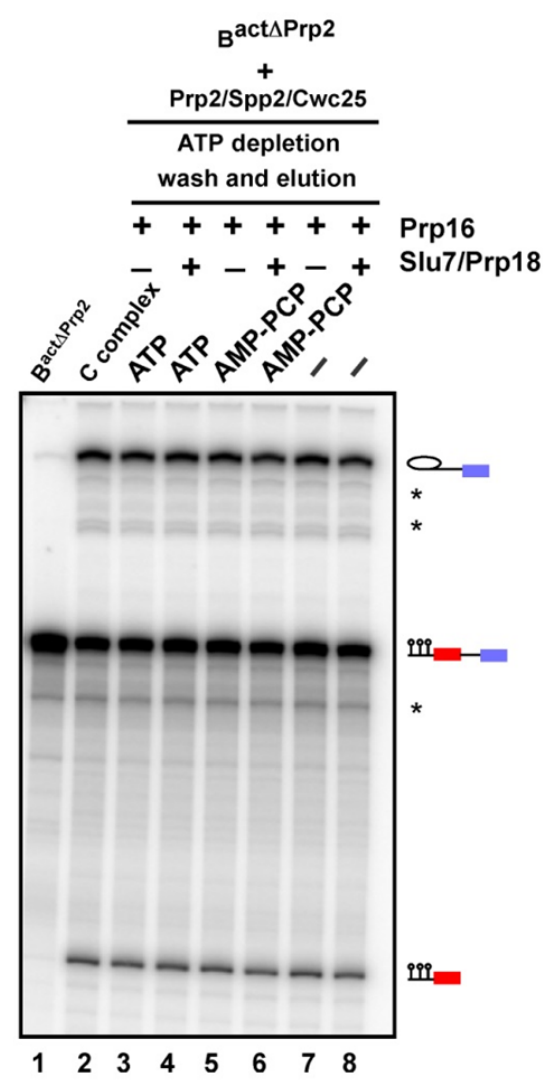

Actwt pre-mRNA

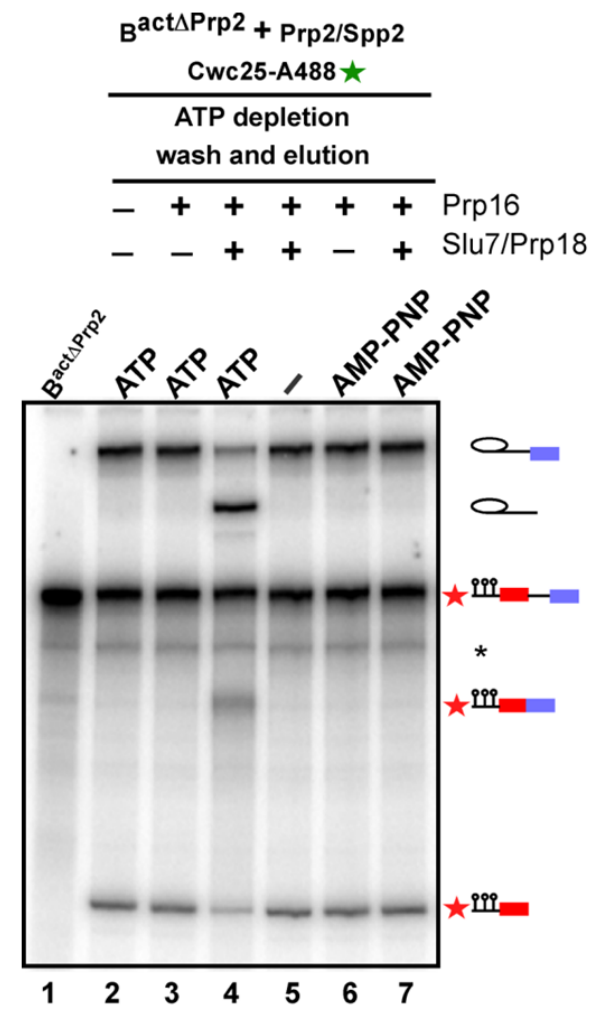

Figure 27: Splicing assay of spliceosomes assembled on ActACAC pre-mRNA and Actwt pre-mRNA. ActACAC and Actwt pre-mRNAs were extracted from spliceosomal complexes obtained as described above each lane, separated by denaturing PAGE, and visualized by autoradiography. The positions of the pre-mRNAs and the splicing intermediates are indicated on the right. Asterisks: uncharacterized pre-mRNA bands. (Figure also appeared in Ohrt et al. $\left.{ }^{84}\right]$ )

the spliceosome and not the recruitment of Slu7/Prp18 to the spliceosome per se. To test this hypothesis, Act pre-mRNA with a shorter distance between the branch site and the 3'SS was used. This experiment is described in the following section.

\subsubsection{Binding of Cwc25-Alexa488 to the Act7wt pre-mRNA}

The requirement of step 2 factors for the second catalytic step is less strict if the distance between the branch site and the 3'SS is short 65 66 [66. Act7 pre-mRNA with a distance of seven nucleotides between the BS and 3'SS was used to investigate the binding behavior of Cwc25 for pre-mRNAs with short distances. The results of 


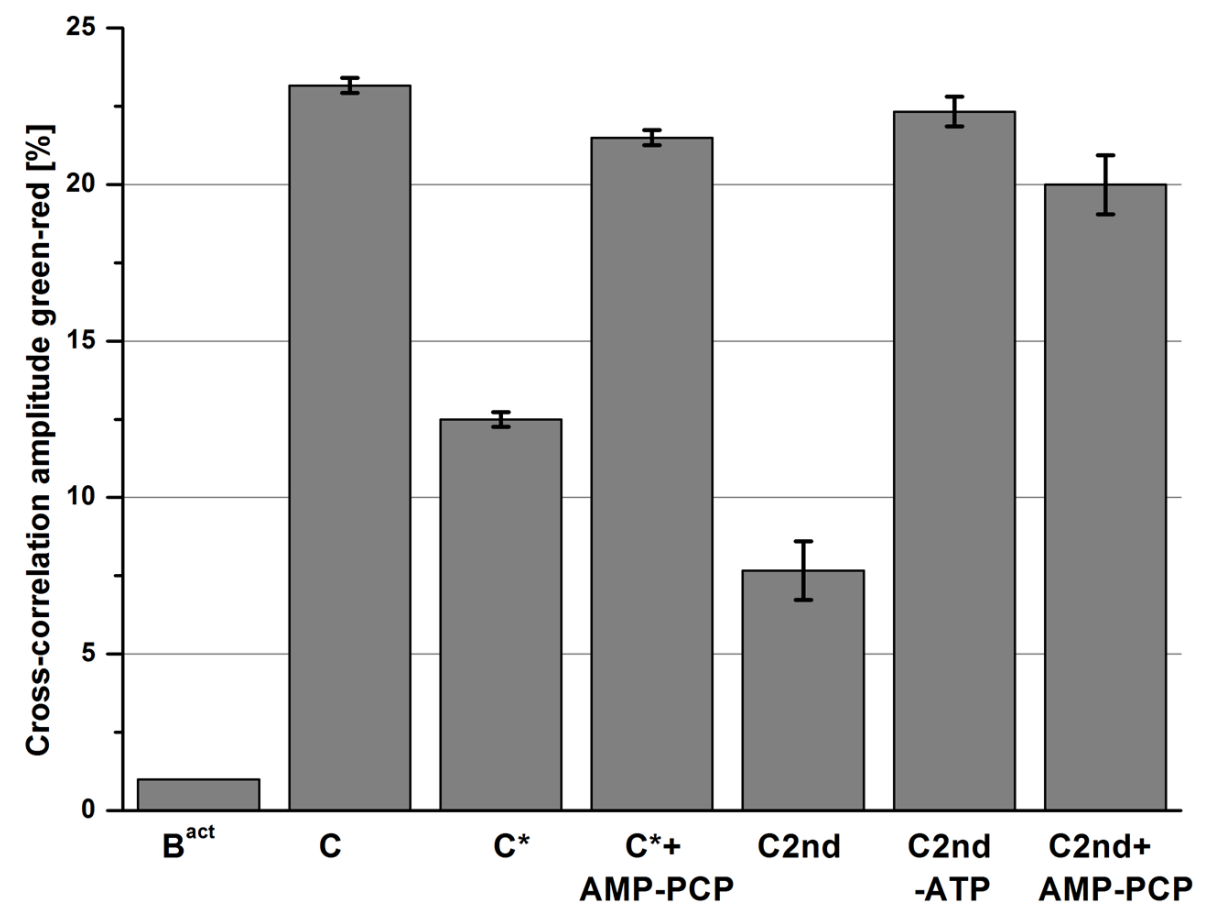

Figure 28: Binding of Cwc25-Alexa488 to Act7wt pre-mRNA. Eluted C complexes were complemented in solution with Prp16 and Slu7/Prp18. FCCS measurements were performed upon the addition of ATP, AMP-PCP, or without ATP. (Figure also appeared in the PhD thesis of Peter Odenwälder and in Ohrt et al. ${ }^{\text {[84] }}$ )

FCCS measurements with Act7 pre-mRNA are shown in figure 28. Comparable to the previous results, Cwc 25 was bound to the $\mathrm{C}$ complex shown by a high crosscorrelation amplitude of $\sim 23 \%$. Further incubation of C complexes with Prp16 and ATP led to a lower cross-correlation amplitude of $12.5 \%$ (see fig. 28, column C and $\mathrm{C}^{*}$ ) which indicates that a high amount of Cwc25 was released. Incubation of C complexes with Prp16, Slu7/Prp18, and ATP induced further release of Cwc25 indicated by a decrease in cross-correlation amplitude to $\sim 7 \%$. Similar to the previous results, incubation with AMP-PCP or without ATP did not change the cross-correlation amplitudes which were about $20 \%$ to $22 \%$. This suggests that Cwc25 does not dissociate from the spliceosome.

To investigate the factor requirements for step 2 catalysis for spliceosomes assembled on Act7 pre-mRNA, affinity-purified Act7-B ${ }^{\text {act }}$ complexes were eluted from the matrix, complemented with recombinant proteins, and analyzed by denaturing gel electrophoresis (see fig. 29). 


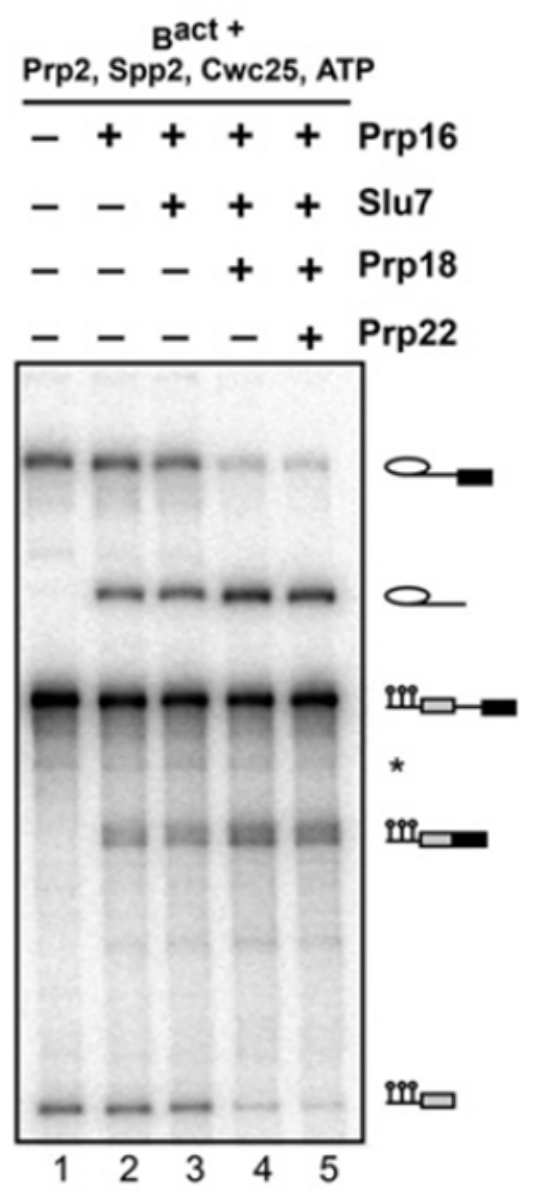

Figure 29: Factor requirements for the second catalytic step of complexes assembled on Act7 pre-mRNA. $B^{\text {act }}$ complexes were complemented with Prp2, Spp2, Cwc25, and ATP (lane 1). Complexes were additionally complemented with Prp16 (lane 2), Slu7/Prp18 (lane 4), and Prp22 (lane 5). (Figure also appeared in the PhD thesis of Peter Odenwälder and in Ohrt et al. ${ }^{84}$ )

Incubation of Act7-B ${ }^{\text {act }}$ complexes with Prp2, Spp2, Cwc25, and ATP yielded efficient step 1 intermediates (see lane 1 in fig. 29). The addition of Prp16 led to the formation of step 2 splicing products (lane 2). Upon further addition of Slu7 and Prp18, the amount of splicing products, i.e. mRNA and intron lariat, was increased (lane 4). The addition of Prp22 had no effect on the amount of splicing products (lane 5).

These data showed that for a pre-mRNA with a short distance between BS and 3'SS, incubation of C complexes with Prp16 and ATP is sufficient for the release of a small amount of Cwc25 from the spliceosome and for catalysis of the second step. Further addition of Slu7/Prp18 led to the dissociation of more Cwc25 and to a higher amount of splicing products. 


\subsubsection{Binding of Cwc25-Alexa488 to the Act7ACAC mutated pre-mRNA}

In the next experiments, spliceosomes were assembled on Act7ACAC pre-mRNA. This pre-mRNA has a distance of seven nucleotides between BS and 3'SS and a mutation on the 3'SS. Due to this mutation, the second catalytic step is blocked. With this experiment it can be tested if the second catalytic step is a prerequisite for the dissociation of Cwc25 from spliceosomes assembled on Act7 pre-mRNA.

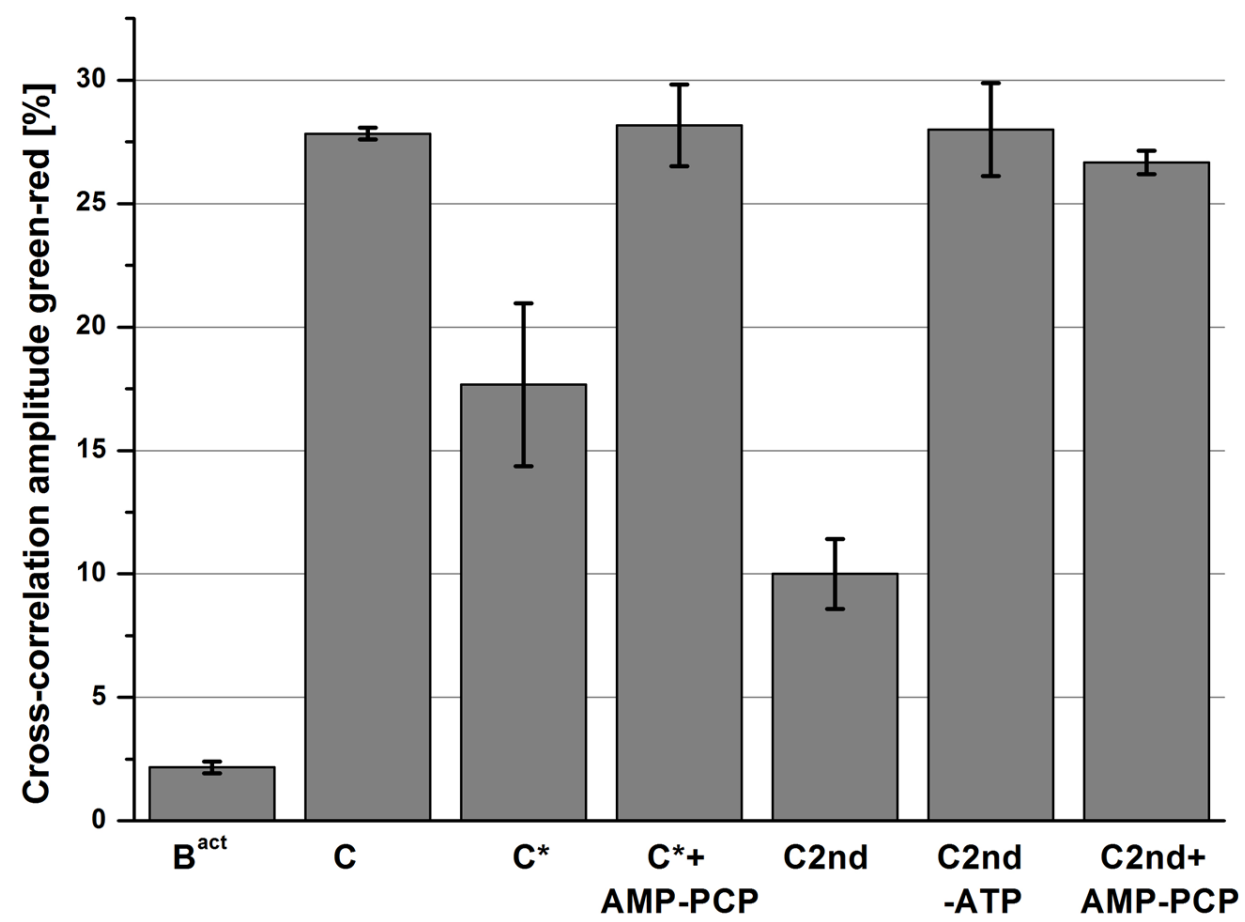

Figure 30: Binding of Cwc25-Alexa488 to Act7ACAC 3'SS mutated premRNA. Eluted C complexes were complemented in solution with Prp16 and Slu7/Prp18. FCCS measurements were performed upon the addition of ATP, AMP-PCP, or without ATP. (Figure also appeared in the PhD thesis of Peter Odenwälder and in Ohrt et al. ${ }^{84]}$ )

The measured cross-correlation amplitudes are shown in figure 30 and were similar to the cross-correlation amplitudes observed for spliceosomes assembled on Act7 pre-mRNA. After incubation of the $\mathrm{C}$ complex with Prp16 and ATP, the crosscorrelation amplitude was decreased from $\sim 27 \%$ to $\sim 17 \%$. A further decrease to $\sim 10 \%$ was observed for the C complex incubated with Prp16, Slu7/Prp18, and ATP. Similar to the previous measurements on different pre-mRNAs, ATP was necessary for the release of Cwc25. Incubation with AMP-PCP or without ATP led to further binding of Cwc25 to the spliceosome. 
The measurements on spliceosomes assembled on Act7ACAC pre-mRNA showed that Cwc25 is released from the spliceosome by the action of Prp16. Further release is initiated by the action of Slu7/Prp18. The second catalytic step per se is again not a condition for the dissociation of Cwc25.

In summary, the results of all measurements on spliceosomes assembled on Act premRNA, ActACAC pre-mRNA, Act7 pre-mRNA, and Act7ACAC pre-mRNA showed that the second catalytic step is not a cause for the release of Cwc25. The distance between the branch site and 3'SS is important and can change the conditions for Cwc25's release. The results are consistent with the assumption that the docking of the 3'SS to the active step 2 site is sufficient for the release of Cwc25. 


\subsection{Functionality testing of Slu7-eGFP and Prp16-eGFP}

The previous FCCS measurements showed that Prp16 and Slu7/Prp18 are involved in the dissociation process of Cwc25. The stage of the spliceosome at which Prp16 and Slu7/Prp18 are recruited to the spliceosome and their binding sites are poorly understood. To clarify these ambiguities, the binding and dissociation of Prp16eGFP and Slu7-eGFP/Prp18 to the different stages of the spliceosome were investigated. For the experiments, a fusion protein of Slu7 with eGFP and a fusion protein of Prp16 with eGFP were expressed in yeast cells. Before starting with the FCCS experiments, the functionality of Slu7-eGFP and Prp16-eGFP was tested by an in vitro splicing assay which is shown in figure 31 .

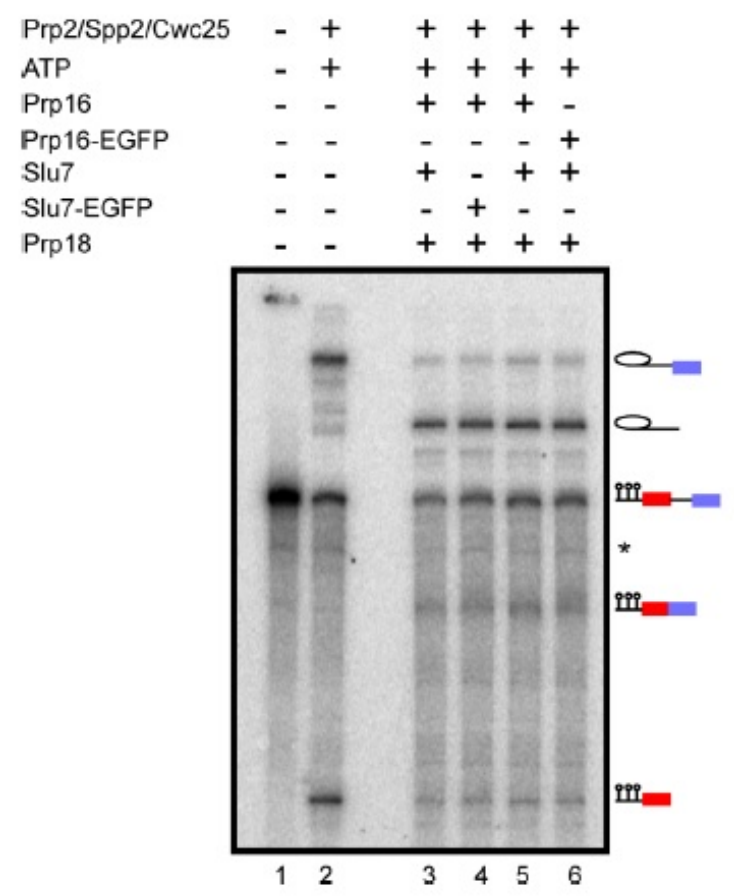

Figure 31: Functional assay for testing the functionality of Slu7-eGFP and Prp16-eGFP. $B^{\text {act }}$ complexes assembled on Actwt pre-mRNA (lane 1) were complemented with Prp2, Spp2, ATP, and Cwc25 (lane 2). Complexes were additionally complemented with Slu7-eGFP (lane 4), Prp16-eGFP (lane 6), or Prp16, Slu7, and Prp18 (lanes 3 and 5). (Figure also appeared in Ohrt et al. ${ }^{84}$ )

The splicing assay showed the splicing products for spliceosomes complemented with Slu7-eGFP (lane 4) instead of Slu7 (lane 3) and Prp16-eGFP (lane 6) instead of Prp16 (lane 5). The bands were similar for the four different compositions and both proteins are functional if they carry the fluorescent protein eGFP. 


\subsection{FCCS measurements for investigating the binding of Slu7- eGFP}

Previous immunoprecipitation studies suggested that Slu7 binds stably to the spliceosome after the Prp16-mediated remodeling of the spliceosome 6165. However, it is unclear if Slu7 already has a binding site at an earlier stage of the splice cycle. To clarify this, the cross-correlation amplitudes for Slu7-eGFP bound to the $\mathrm{B}^{\text {act }}, \mathrm{B}^{*}$, $\mathrm{C}$, and $\mathrm{C}^{*}$ complex at low salt $(75 \mathrm{mM} \mathrm{KCl})$ and high salt $(150 \mathrm{mM} \mathrm{KCl})$ conditions were measured. The different complexes were reconstituted by supplementing affinity-purified $B^{\text {act }}$ on the amylose affinity matrix with recombinant splicing factors. Then, the complexes were incubated with Slu7-eGFP and Prp18, and without Prp18 and washed with GK75 buffer respectively GK150 buffer. In the end, the complexes were eluted from the matrices with maltose and measured with FCCS. The results are shown in figure 32 .

First, the cross-correlation amplitudes for complexes which were washed with a low salt buffer containing $75 \mathrm{mM} \mathrm{KCl}$ were measured. The observed cross-correlation amplitude for $\mathrm{B}^{\text {act }}$ complexes incubated with Slu7 and Prp18 was low ( 3\%) and Slu7-eGFP was not bound to the $\mathrm{B}^{\text {act }}$ complex. After the addition of Prp2/Spp2, the cross-correlation amplitude was increased up to $22 \%$ and Slu7 was bound efficiently to $\mathrm{B}^{*}$ complexes (see fig. 32, column 2).

In order to form the $\mathrm{C}$ complex, the $\mathrm{B}^{\text {act }}$ complex was supplemented with Prp2/Spp2, Cwc25, and ATP. Further addition of Prp16 led to the formation of the $\mathrm{C}^{*}$ complex. The addition of Slu7 and Prp18 to the $\mathrm{C}^{*}$ complex then resulted in a post-catalytic (PC) complex. The obtained cross-correlation amplitudes for the $\mathrm{C}$ and $\mathrm{C}^{*} / \mathrm{PC}$ complex were comparably high (18\% - $19 \%)$.

These data showed that Slu7 was bound to the spliceosome at an early stage. A binding site for Slu7/Prp18 is already created when the spliceosome is catalytically activated by Prp2, Spp2, and ATP. Without Prp18, the measured cross-correlation amplitude was low $(\sim 2 \%)$ and Slu7 is only bound to the spliceosome in the presence of Prp18. This result is consistent with the assumption that Slu7 and Prp18 bind as a heterodimer to the spliceosome.

In the next step, the spliceosomes were washed with GK150 buffer with $150 \mathrm{mM}$ $\mathrm{KCl}$ instead of $75 \mathrm{mM} \mathrm{KCl}$. The resulting cross-correlation amplitudes are shown in figure 32 on the right side. In comparison to complexes washed with $75 \mathrm{mM} \mathrm{KCl}$, the cross-correlation amplitudes for $\mathrm{B}^{\text {act }}, \mathrm{B}^{*}$, and $\mathrm{C}$ complexes were low $(\sim 1 \%)$. Slu7 was not bound to these complexes. The cross-correlation amplitude was high 

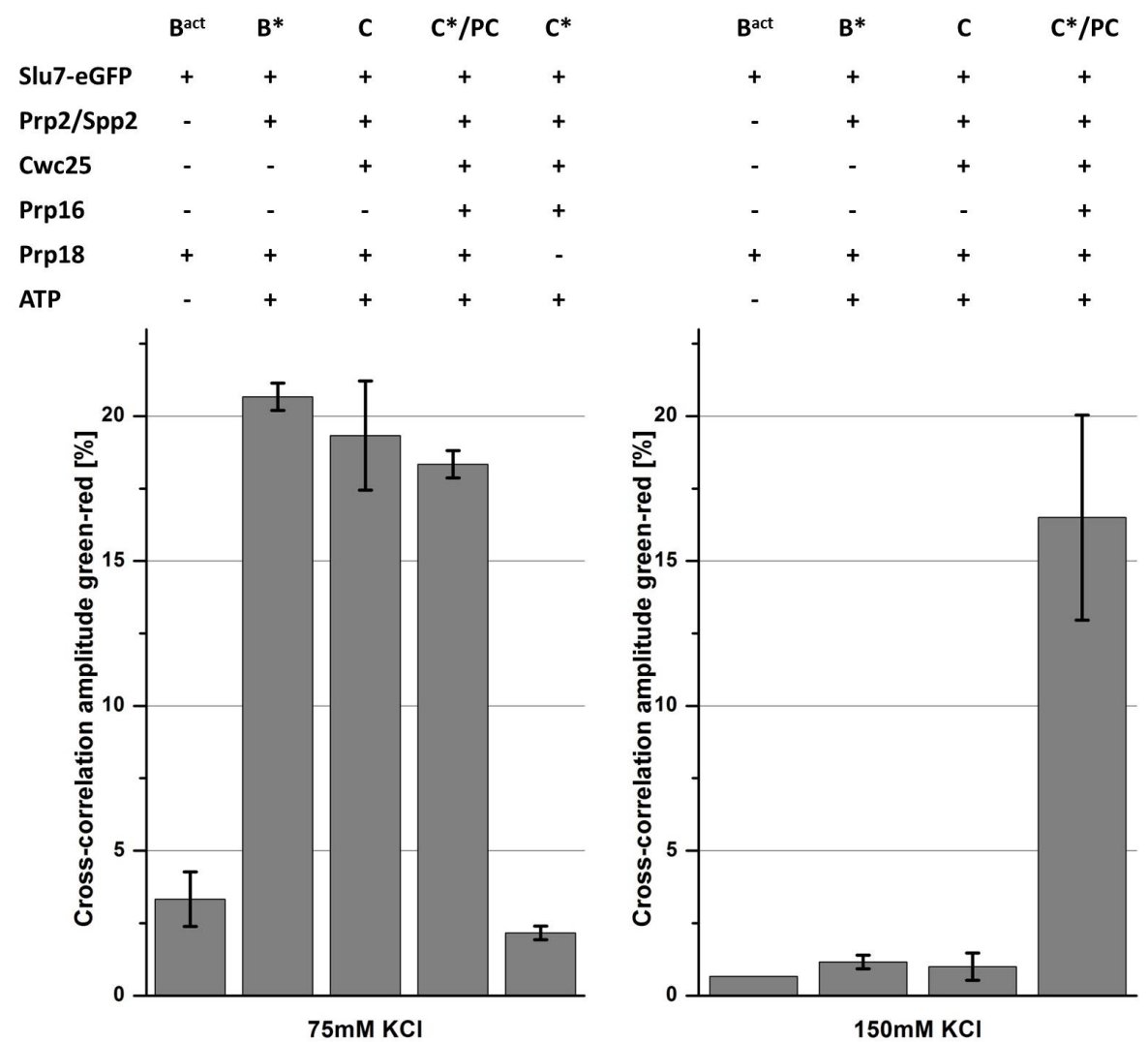

Figure 32: Binding behavior of Slu7-eGFP to the spliceosome. Cross-correlation amplitudes for the binding of Slu7-eGFP to the $B^{\text {act }}, B^{*}, C$, and $C^{*} / P C$ complexes are shown. $C^{*} / P C$ indicates that upon Slu7/Prp18 binding to the $C^{*}$ complex, the latter is transformed into the post-catalytic complex $(P C)$. Measurements were performed with $75 \mathrm{mM} \mathrm{KCl}$ and $150 \mathrm{mM} \mathrm{KCl}$. (Figure also published in Ohrt et al. ${ }^{84}$ )

( 16 \%) when the $\mathrm{B}^{\text {act }}$ complex was incubated with Prp2/Spp2, Cwc25, Prp16, Prp18, ATP, and Slu7 .

For testing if C complexes containing Slu7/Prp18 catalyze the second catalytic step, a denaturing PAGE was performed. Reconstituted $\mathrm{C}$ complexes were incubated on the matrix with Slu7/Prp18 at $75 \mathrm{mM} \mathrm{KCl}$ and $150 \mathrm{mM} \mathrm{KCl}$. Complexes were washed with GK75 buffer and GK150 buffer, respectively. Then they were eluted from the matrix in GK75 buffer and mock-treated or incubated with Prp16. After incubation, the RNAs were extracted and separated by denaturing PAGE. The RNAs from mock-treated complexes are shown in lanes 2 and 5 in figure 33 and RNAs from complexes incubated with Prp16 plus ATP in lanes 3 and 6 . This experiment shows that $\mathrm{C}$ complexes which were washed with GK75 buffer and thus have a low-affinity binding site for Slu7/Prp18, catalyze the second step efficiently if Prp16 and ATP are present (see lane 3 in fig. 33). Splicing products were not 


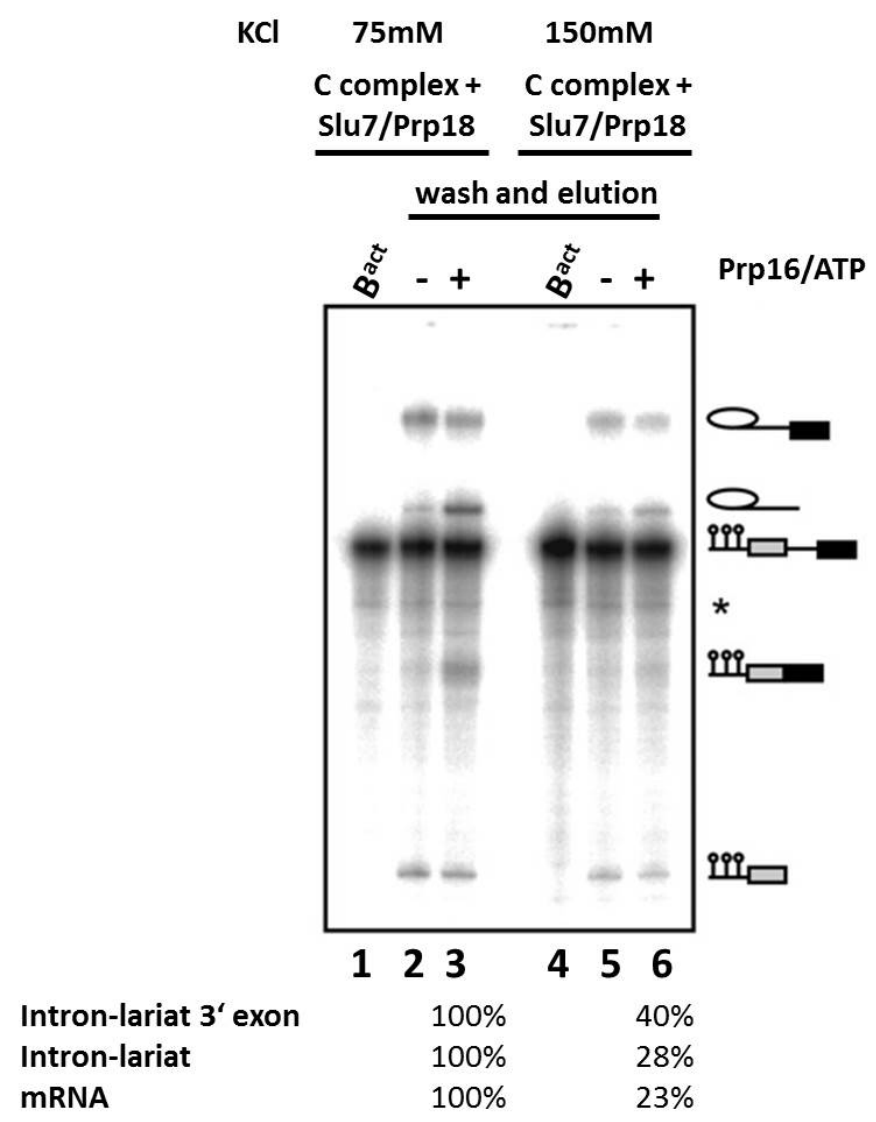

Figure 33: Denaturing PAGE with $\boldsymbol{C}$ complexes. Denaturing PAGE of extracted $R N A$ s of C complexes which were washed with buffers containing 75 or $150 \mathrm{mM} \mathrm{KCl}$, eluted from the matrix, and mock-treated or incubated with Prp16 plus ATP. Using ImageQuant software, the intensities of the intermediate and mRNA product signals were quantified.

The signals in lane 3 were used as $100 \%$. (Figure also appeared in Ohrt et al. ${ }^{[84}$ )

detected for the $\mathrm{B}^{\text {act }}$ complex or the $\mathrm{C}$ complex without Prp16 plus ATP.

If the C complex was washed with GK150 buffer, only low amounts of mRNA products were detected (see lane 6 in fig. 33). By quantifying the signal intensities of the intermediates and mRNA products by using the ImageQuant software (Molecular Dynamics) and setting the signals in lane 3 as $100 \%$, the amounts of mRNA products in lane 6 can be calculated. C complexes washed with GK150 buffer yielded only $40 \%$ of the intron-lariat 3' exon, $28 \%$ of the intron-lariat, and $23 \%$ of mRNA. These measurements showed that the binding affinity of Slu7/Prp18 to the $\mathrm{B}^{\text {act }}, \mathrm{B}^{*}$, and $\mathrm{C}$ complex is low because the observed cross-correlation amplitudes were low at harsh conditions, i.e. $150 \mathrm{mM} \mathrm{KCl}$. Due to the high cross-correlation amplitude obtained for $\mathrm{C}^{*}$ complexes at low and high salt concentrations, the low-affinity binding site is converted into a high-affinity binding site for the $\mathrm{C}^{*} / \mathrm{PC}$ complex. 
The conversion into a high-affinity binding site is done by the Prp16 ATP-dependent remodeling of the $\mathrm{C}$ complex into the $\mathrm{C}^{*}$ complex.

The data obtained from the electrophoresis experiment suggest that Slu7/Prp18 can facilitate the second catalytic step if it is bound to its low-affinity binding site in the $\mathrm{C}$ complex. This indicates that the low-affinity binding site is the functionally relevant site.

\subsection{FCCS measurements for investigating the binding of Prp16- eGFP}

It was shown that Prp16 may act during step 1 of splicing ${ }^{8788}$. To prove this assumption, FCCS measurements with Prp16 similar to the experiments with Slu7 were performed. For the experiments, Prp16-eGFP was overexpressed in yeast cells and subsequently purified as described in the method section. The binding behavior of Prp16-eGFP to the $\mathrm{B}^{\text {act }}, \mathrm{B}^{*}, \mathrm{C}^{*}$, and $\mathrm{C}^{*} / \mathrm{PC}$ complex at low salt concentration $(75 \mathrm{mM} \mathrm{KCl})$ and at high salt concentration $(150 \mathrm{mM} \mathrm{KCl})$ was observed.

First, the binding of Prp16 to the spliceosomal complexes at low salt concentration was studied. The obtained data are shown on the left side in figure 34 . For the $\mathrm{B}^{\text {act }}$ complex, a cross-correlation amplitude of $\sim 7 \%$ was measured (see fig. 34 , column 1) which indicates that Prp16 was not bound to this complex. After the remodeling of the spliceosome activated by Prp2, Prp16 was bound to the $\mathrm{B}^{*}$ complex which could be seen by a high cross-correlation amplitude of $\sim 25 \%$. A comparable crosscorrelation amplitude was observed for the $\mathrm{C}^{*}$ complex after the addition of Cwc25. After further addition of Slu7/Prp18 and the conversion of the $\mathrm{C}^{*}$ complex into the $\mathrm{C}^{*} / \mathrm{PC}$ complex by the action of Prp16 and ATP, the cross-correlation amplitude remained high $(\sim 27 \%)$. These results indicate that Prp16 is bound to the $\mathrm{B}^{*}, \mathrm{C}^{*}$, and $\mathrm{C}^{*} / \mathrm{PC}$ complex.

When the experiment was carried out at high salt concentration $(150 \mathrm{mM} \mathrm{KCl})$, the cross-correlation amplitude for the $\mathrm{B}^{\text {act }}$ complex remained low $(\sim 3 \%)$ (see fig. 34 . right side). For the $\mathrm{B}^{*}$ complex, the observed cross-correlation amplitude of $\sim 15 \%$ was lower than the one for the $\mathrm{B}^{*}$ complex at $75 \mathrm{mM} \mathrm{KCl}(\sim 25 \%)$. However, the cross-correlation amplitude was still higher than for the $\mathrm{B}^{\text {act }}$ complex and thereby higher than the background. The measured cross-correlation amplitude for the $\mathrm{C}^{*}$ complex was high $(\sim 25 \%)$ and similar to the observed cross-correlation amplitude for the $\mathrm{C}^{*}$ complex at low salt concentration. From the high cross-correlation amplitude even at harsh conditions it could be concluded that Prp16 has a high binding affinity 


$\begin{array}{lcccccccc} & B^{\text {act }} & \mathrm{B}^{*} & \mathrm{C}^{*} & \mathrm{C}^{*} / \mathrm{PC} & \mathrm{B}^{\text {act }} & \mathrm{B}^{*} & \mathrm{C}^{*} & \mathrm{C}^{*} / \mathrm{PC} \\ \text { Prp16-eGFP } & + & + & + & + & + & + & + & + \\ \text { Prp2/Spp2 } & - & + & + & + & - & + & + & + \\ \text { CWC25 } & - & - & + & + & - & - & + & + \\ \text { Slu7/Prp18 } & - & - & - & + & - & - & - & + \\ \text { ATP } & + & + & + & + & + & + & + & +\end{array}$
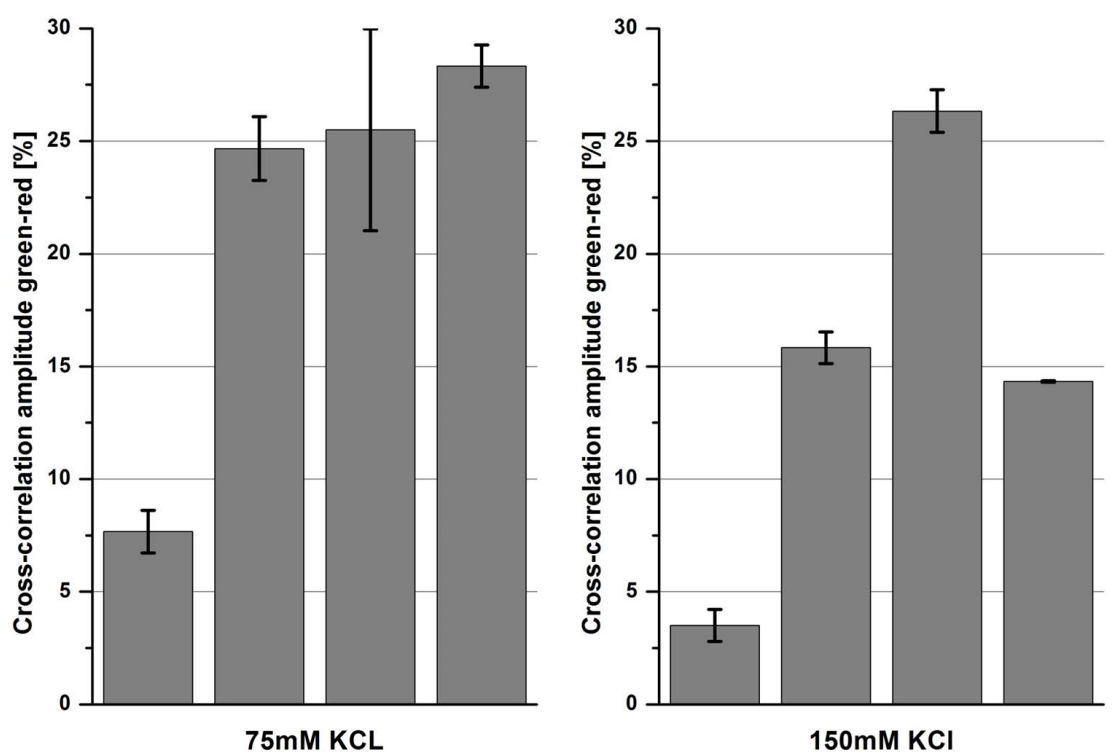

Figure 34: Binding behavior of Prp16-eGFP to the spliceosome. Cross-correlation amplitudes for the binding of Prp16-eGFP to the $B^{\text {act }}, B^{*}, C^{*}$, and $C^{*} / P C$ complexes are shown. $C^{*} / P C$ indicates that upon Slu7/Prp18 binding to the $C^{*}$ complex, the latter is transformed into the post-catalytic complex $(P C)$. Measurements were performed with $75 \mathrm{mM} \mathrm{KCl}$ and $150 \mathrm{mM} \mathrm{KCl}$. (Figure also published in Ohrt et al. ${ }^{84}$ )

to the $\mathrm{C}^{*}$ complex. For the $\mathrm{C}^{*} / \mathrm{PC}$ complex, the cross-correlation amplitude was reduced to $\sim 15 \%$.

In summary, Prp16 has already a binding site on the B* complex. The affinity of Prp16 is more salt resistant compared to Slu7/Prp18 and Prp16 is released from the spliceosome after the second catalytic step has proceed. 


\subsection{Discrimination of Prp16's and Slu7's binding site on the B* spliceosome}

The measurements on Slu7-eGFP and Prp16-eGFP showed that both proteins have binding sites on the $\mathrm{B}^{*}$ complex. It is still unclear if they have the same binding site, different binding sites, or partially overlapping sites. To answer this question, FCCS was used to monitor the binding of Prp16-eGFP to the B* complex after the addition of different amounts of unlabeled Prp16 and unlabeled Slu7/Prp18. The observed cross-correlation amplitudes are shown in figure 35.

By increasing the amount of unlabeled Prp16 (1:1, 1:3, 1:7 of Prp16-eGFP to Prp16), the cross-correlation amplitudes were reduced from $26 \%$ to $13 \%$ (see fig. 35, column 1-4). By increasing the amount of unlabeled Slu7/Prp18, the cross-correlation amplitudes remained constant at $\sim 25 \%$. This corresponds to a high binding affinity of Prp16-eGFP to the $\mathrm{B}^{*}$ complex.

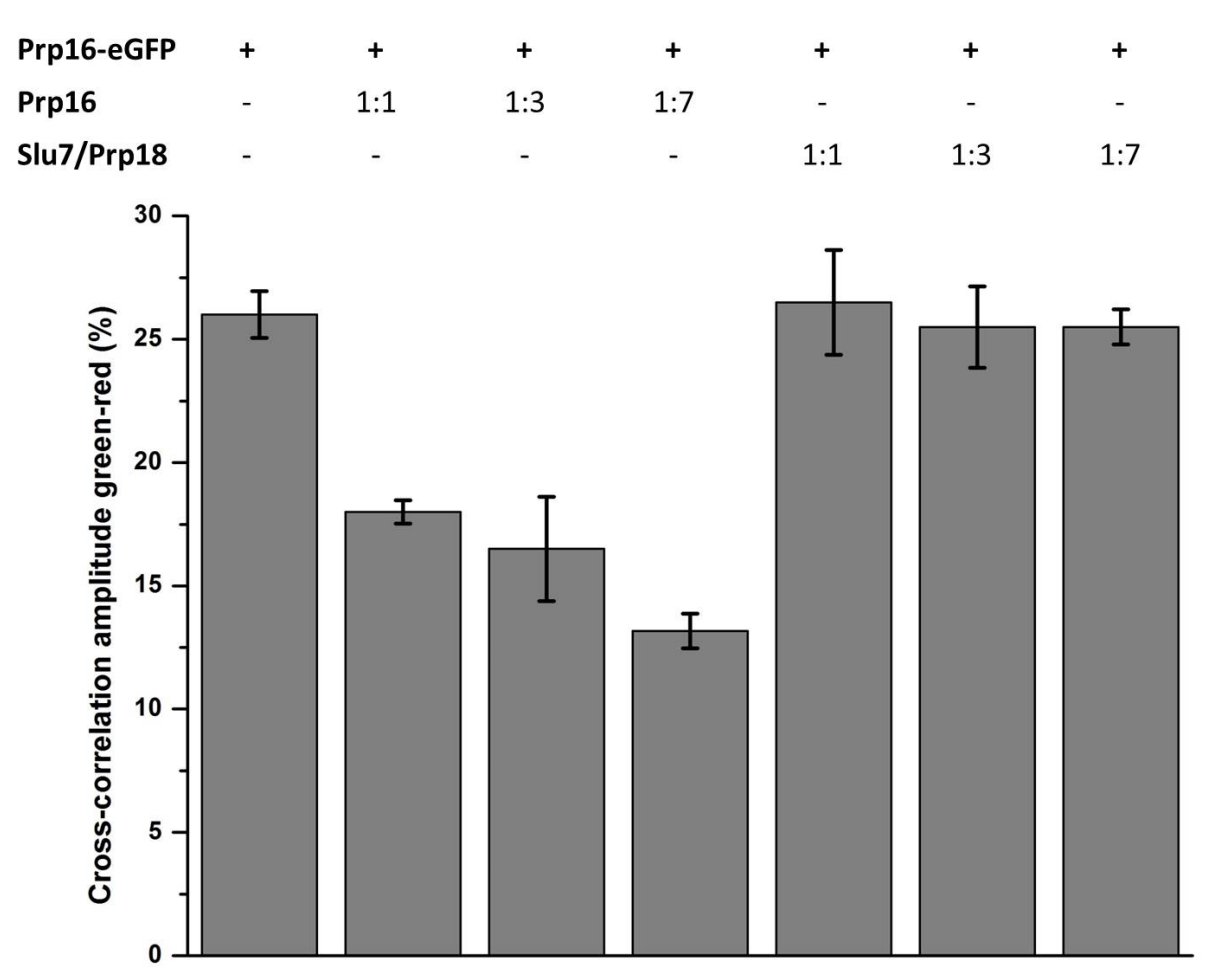

Figure 35: Competition of Prp16-eGFPs binding site with the one for Slu7/Prp18. Cross-correlation amplitudes for the binding of Prp16-eGFP to the $B^{*}$ spliceosome after the addition of unlabeled Prp16 or Slu7/Prp18 in different amounts. The amount of unlabeled Prp16 or Slu7/Prp18 was increased in the ratio of 1:1, 1:3, and 1:7 of Prp16-eGFP to Prp16 or Slu7/Prp18. (Figure also published in Ohrt et al. ${ }^{84}$ ) 
In the next step, the complementary experiment was performed by monitoring the binding behavior of Slu7-eGFP after the addition of increasing amounts of unlabeled Slu7/Prp18 or unlabeled Prp16. The results are shown in figure 36.

Comparable to the experiment with Prp16, the cross-correlation amplitude was decreased after the addition of unlabeled Slu7/Prp18 to Slu7-eGFP from 17\% to 6\% (1:1, 1:3, 1:7 of Slu7-eGFP to Slu7/Prp18). The cross-correlation amplitudes remained constant after the addition of unlabeled Prp16 at $15 \%$.

The data of both experiments indicate that Prp16 and Slu7/Prp18 have different binding sites on the catalytically activated $\mathrm{B}^{*}$ complex. Otherwise, the crosscorrelation amplitude of Slu7-eGFP to the B* complex should show a decrease after the addition of Prp16 and vice versa.

$\begin{array}{lccccccc}\text { Slu7-eGFP } & + & + & + & + & + & + & + \\ \text { Prp18 } & + & + & + & + & + & + & + \\ \text { Slu7/Prp18 } & - & 1: 1 & 1: 3 & 1: 7 & - & - & - \\ \text { Prp16 } & - & - & - & - & 1: 1 & 1: 3 & 1: 7\end{array}$

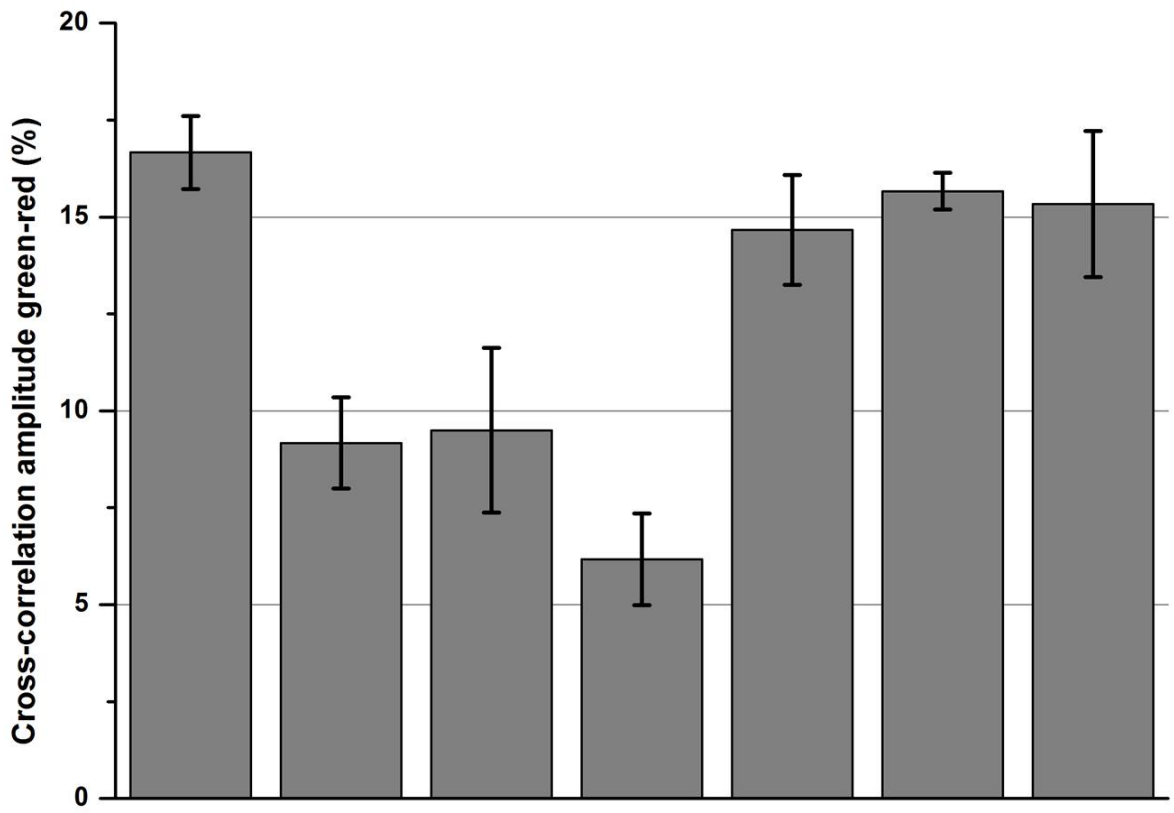

Figure 36: Competition of Slu7-eGFPs binding site with the one for Prp16. Cross-correlation amplitudes for the binding of Slu7-eGFP/Prp18 to the $B^{*}$ spliceosome after the addition of Prp18 and different amounts of unlabeled Prp16 or Slu7/Prp18. The amount of unlabeled Slu7/Prp18 was increased in the ratio of 1:1, 1:3, and 1:7 of Slu7-eGFP to Prp16 or Slu7/Prp18.

(Figure also published in Ohrt et al. ${ }^{84}$ ) 


\section{Discussion}

\subsection{FCCS studies on the spliceosome}

FCCS is a method to study interactions of various molecules such as proteins, lipids, or carbohydrates, in solution with the need of being fluorescent either by carrying already a natural fluorescent center or by a fluorescent tag attached to it. In this study, FCCS was used to monitor binding reactions and interactions of proteins to the spliceosome, a huge macromolecular complex.

The spliceosome, added proteins and reaction conditions have to fulfill several criteria during the experiment. First, the spliceosome needs to be stable over the preparation and measurement time (3 hours), and degradation and aggregation of the complexes have to be avoided as they can distort the correlation curves. Second, it has to be tested if the spliceosomes are stable at the nanomolar concentrations. Nanomolar concentrations are necessary because FCCS is a single molecule method. The feasibility of FCCS for studying dynamics of the spliceosomes was investigated. The temporal stability was tested with purified labeled spliceosomes carrying an additional fluorescently labeled Cwc22 protein over a time range of 3 hours. Cwc22 was used to investigate the temporal stability because it is not a core component of the spliceosome 37 . The cross-correlation amplitudes for Cwc22's binding to the $\mathrm{B}^{\text {act }}$ complex at time point zero and after 3 hours were comparably high (see fig. 20 ). The shape of the correlation curves and their perfect fits showed that the sample did not aggregate or degrade over 3 hours (see fig. 21). The measurements showed further that no additional fluorescent background is generated by the spliceosome or the used buffers. In addition, the fluorescent signals were constant over the measurement time and no amplitude due to aggregates could be observed in the count rates (data not shown).

The purified spliceosomal complexes have to behave in the same way in all FCCS measurements, and the addition of further proteins should not induce further aggregation of the complexes. These criteria were investigated by using Snu114-eGFP because Snu114 is a stable component during the activation and catalytic steps of the spliceosome. The observed cross-correlation amplitudes were constant at the different maturation steps of the spliceosome (see fig. 22). Snu114-eGFP remained stably attached to all complexes as observed for native Snu114 proteins in previous studies 82 and no dissociation of proteins due to the necessary low concentrations ( $1 \mathrm{nM}$ for single molecule measurements) was observed. For inducing the activation 
and catalytic steps, several proteins were added to the solution. These proteins induced no aggregation of complexes and had no further disturbing effects because the cross-correlation curves were all well-defined (data not shown). The measurements showed further that the fluorescent labels did not induce artefacts in the FCCS measurements because Snu114-eGFP and native Snu114 showed a similar binding behavior.

To test whether the fluorescent labels have an effect on the spliceosome behavior and thereby inducing artefacts, reconstitution assays where performed with extracts from tagged yeast strains or tested in reconstituted splicing assays, e.g. Prp16-eGFP and Slu7-eGFP (fig. 31). The reconstitution assay showed that the labeled proteins were just as functional as native proteins and the catalysis of the spliceosome was not influenced by the fluorophores.

To investigate the suitability of FCCS for observing small changes in the binding affinity of a protein, the measurement errors have to be considered. For example, measurements on Snu114-eGFP and the $\mathrm{B}^{\text {act } \Delta \operatorname{Prp} 2}, \mathrm{~B}^{*}, \mathrm{C}, \mathrm{C}^{*}$, and $\mathrm{C} 2$ nd complex yielded high cross-correlation amplitudes of around $57 \%$ (see fig. 22). The standard deviations for the data points were between $0.2 \%$ to $1.4 \%$ and comparable to the cross-correlation amplitudes quite low. The cross-correlation amplitudes for Snu114, which is bound to all complexes, only differed in a range of $2 \%$. Therefore, FCCS can be used for monitoring small changes in the binding of a protein to the spliceosome.

\subsubsection{Information about binding affinities given by FCCS}

Accurately describing protein function and activity is still difficult. FCCS offers a possibility for determining protein affinities with high precision and is thus a promising tool to tackle this problem. In most studies, the binding interactions between small molecules were investigated ${ }^{20 \mid 22}$, but no study monitored the binding constant in a macromolecular complex in which more than 80 proteins are involved like the spliceosomal $\mathrm{B}^{\text {act }}$ or $\mathrm{B}^{*}$ complex. Here, FCCS was used for the first time to determine a binding constant for the spliceosome in equilibrium and in a nanomolar to picomolar range. The binding affinity between Cwc25, a small protein, and the $\mathrm{B}^{\text {act }}$ and $\mathrm{B}^{*}$ complex were determined. These complexes were chosen because FCCS measurements showed that Cwc25 does not bind efficiently to the spliceosome before the catalytic activation by Prp2 but after the activation.

In the measurements, the standard deviations of several data points were high compared to the cross-correlation amplitude itself (see fig. 25). For $0.01 \mathrm{nM}$ of the $\mathrm{B}^{*}$ 
complex, a cross-correlation amplitude of $3.2 \%$ with a standard deviation of $2.8 \%$ was measured. The standard deviations of other data points were low, e.g. a crosscorrelation amplitude of $11.2 \%$ with a standard deviation of $0.2 \%$ was measured for $0.4 \mathrm{nM} \mathrm{B*}$ complex. These different and partly high standard deviations can influence the fitting procedure, but were taken into account by using a bootstrapping method, which considers the different standard deviations of each data point. The Hill equation fit together with the bootstrapping method yielded a binding constant of $K_{d}=(0.03 \pm 0.01) \mathrm{nM}$. The error of the binding constant is quite high compared to the binding constant itself because the error is influenced by the relatively high standard deviations of particular data points. However, even considering the large error, the binding constant is still low and reflects a strong binding site for Cwc25 on the $\mathrm{B}^{*}$ complex. The relatively large error of the binding constant is also caused by the complexity of the preparation method and the complexity of the sample itself. Additionally, measuring at only picomolar concentration further increases the pipetting error and errors in serial dilutions. Despite the relatively large errors, FCCS can be reliably used to determine binding constants. This was done here for the first time at only picomolar concentrations which is extremely beneficial for biological samples as preparing them in large amounts is very challenging. 


\subsection{Information obtained by FCCS about the recruitment and release of spliceosomal factors}

\subsubsection{Binding behavior of the step 1 factor Cwc25}

FCCS was used to understand the dynamic nature of the spliceosome during its catalytic activation.

Cwc25's binding and release was investigated at the different maturation steps of the spliceosome with a wildtype pre-mRNA. It was observed that Cwc25 was not bound to the $\mathrm{B}^{\text {act }}$ complex shown by a low cross-correlation amplitude which was comparable to the background. The measurement of the $\mathrm{C}$ complex yielded a much higher cross-correlation amplitude than for the $\mathrm{B}^{\text {act }}$ complex (see fig. 24). Thus, by the action of the helicase Prp2 and its cofactor Spp2, a strong binding site for Cwc25 is formed in the $\mathrm{B}^{*}$ complex. The formation of the binding site was also shown by the binding constant measurement on the $\mathrm{B}^{\text {act }}$ and $\mathrm{B}^{*}$ complex (see fig. 25) which yielded for Cwc25-Alexa488 a binding constant of $30 \mathrm{pM}$ to the $\mathrm{B}^{*}$ complex (see fig 25). This value reflects a strong binding of Cwc25-Alexa488 to the $\mathrm{B}^{*}$ complex. In contrast to the high binding affinity to the $\mathrm{B}^{*}$ complex, it was not possible to detect binding of Cwc25-Alexa488 to the $\mathrm{B}^{\text {act }}$ complex. This is in agreement with previous mass spectrometry data of purified spliceosomes showing no Cwc25 at $\mathrm{B}^{\text {act }}$ level and higher values at $\mathrm{C}$ complex 34 . By Prp2s action, a binding site for Cwc25 is generated.

Further incubation of the C complex with Prp16 and ATP yielded the $\mathrm{C}^{*}$ complex. The cross-correlation amplitude was slightly decreased in comparison to the one for the C complex, but no significant change within the error bars for both complexes was observed. The control experiment with Prp16 and AMP-PNP yielded a cross-correlation amplitude that was comparable to the one obtained for $\mathrm{C}$ complexes. This indicates that Prp16 and its ATP-dependent catalytic activation of the spliceosome for step 2 catalysis are not sufficient to release Cwc25 under physiological conditions. This measurement showed a different behavior than the study of Tseng et al., in which Cwc25 is released from the spliceosome after the action of Prp16 solely 69 .

Supplementation of the $\mathrm{C}^{*}$ complex with Slu7/Prp18 led to the formation of the $\mathrm{C} 2$ nd complex and resulted in the significant release of Cwc25. As negative controls for the ATP-dependent action of Prp16, experiments with AMP-PNP instead of ATP or without ATP were performed. In the absence of ATP, a decrease comparable to 
the one for the C2nd complex formation could not be observed. To proof that Prp16 without ATP hydrolysis is not able to form the catalytic center for step 2 catalysis, the measurement was performed in the presence of AMP-PNP (a non-hydrolysable ATP analog). Again, the cross-correlation amplitude remained at a high level and no release of Cwc25 could be observed. This indicates that in both cases, i.e. absence of ATP and presence of AMP-PNP, the $\mathrm{C}^{*}$ complex formation and thereby also the $\mathrm{C} 2 \mathrm{nd}$ complex formation were inhibited.

The measurements on complexes supplemented on Actwt pre-mRNA showed that the ATPase activity of Prp16 and Slu7/Prp18 are necessary for the dissociation of Cwc25 from the spliceosome. However, it is unclear if the binding of Slu7/Prp18, the second catalytic step, or the docking of the 3'SS into the catalytic center is responsible for Cwc25s dissociation. To determine if the 2nd catalytic step or the docking of the 3'SS induce Cwc25's release, spliceosomes were assembled on ActACAC pre-mRNA which is incapable of undergoing the second catalytic step due to the mutation in the 3'SS. The results of these measurements were comparable to the results of the FCCS measurements on complexes assembled on Actwt premRNAs (see fig. 26) and indicate that even when the spliceosome cannot undergo the second catalytic step, Slu7/Prp18 and the activity of Prp16 are necessary for the release of Cwc25. The FCCS experiments on Actwt pre-mRNA and ActACAC pre-mRNA suggest that the binding of Slu7/Prp18 to the spliceosome or a conformational change induced by their binding directly displaces Cwc25 and not the step 2 catalysis.

To clarify the role of the 3'SS in the release of Cwc25, experiments with spliceosomes assembled on Act7 pre-mRNA were performed. Previous studies showed that the requirement for step 2 factors is less strict if the distance between the branch site and the 3'SS is short 65 [66. A6. An Act7 pre-mRNA with a short distance of seven nucleotides between the BS and 3'SS was used to investigate the binding behavior of Cwc25. Electrophoresis experiments showed that step 2 catalysis of spliceosomes assembled on Act7 pre-mRNA was catalyzed in the absence of Slu7/Prp18, but the step 2 efficiency was increased twofold in the presence of this heterodimer (see fig. 29). However, only Prp16 and ATP are required for exon ligation and not Slu7 or Prp18. Previous studies showed that despite single or double depletions of Slu7, Prp18, or Prp22 from splicing extracts the second catalytic step proceeds 65 66] 66] [56. With the used reconstitution assays, it could be shown that the ATP-dependent action of Prp16 is sufficient for the formation of a functional step 2 site. Exon ligation occurs without Slu7, Prp18, and Prp22. This indicates that for pre-mRNAs with 
a short distance between the BS and 3'SS, diffusion brings the 3'SS to its docking position in the catalytic center and no further help by Slu7/Prp18 is necessary. The explanation for the fact that the addition of Slu7/Prp18 increases the efficiency of the second step could be that the docking of the 3'SS is more efficient in the presence of Slu7/Prp18. This suggests that the action of Slu7/Prp18 alters the conformation of the spliceosome. For pre-mRNAs with a longer distance between BS and 3'SS, the docking of the 3'SS only by diffusion through space is inefficient and may be prevented by secondary structures in the pre-mRNA 89 . Then, Slu7/Prp18 are essential for the second catalytic step and the efficient docking of the 3'SS in the catalytic center. The results with Act7 pre-mRNA showed that the 3'SS is recognized and bound by the step 2 active site without Slu7, Prp18, and Prp22. A previous study demonstrated that in the absence of the step 2 factors the spliced Act7 mRNA is stably bound to the spliceosome of the second step and dissociates only by the action of Prp22 [90. Loop 1 of the U5 snRNA and Prp8 are the known spliceosomal components that are involved in the recognition and binding of the 3'SS 919293 94 95. The function of Slu7/Prp18 could be to help to stabilize the interaction between the 3'SS and the active site. The stabilization may occur directly or indirectly via Prp8, particularly if the interaction of the 3'SS is weak ${ }^{96}$.

In the FCCS experiments on spliceosomes assembled on Act7 pre-mRNA, the crosscorrelation amplitude for the $\mathrm{C}$ complex incubated with Prp16 was reduced and Cwc25 dissociated from the spliceosome in the absence of Slu7/Prp18. The amount of released Cwc25 proteins was increased upon incubation with Prp16, Slu7/Prp18, and ATP (see fig. 28). This result indicates the following mechanism: First, local rearrangement of the catalytic center from the step 1 to step 2 conformation was induced by the ATP-dependent action of Prp16. This structural change decreases the binding affinity of Cwc25 in the spliceosome but Cwc25 is still bound. By the docking of the 3'SS into the active center, Cwc25 is displaced from the spliceosome via an additional conformational change at the catalytic center.

To check if the stable docking of the 3'SS and not the process of the second catalytic step is the trigger for Cwc25s release, experiments with spliceosomes assembled on Act7ACAC pre-mRNA were conducted. The measurements on Act7 pre-mRNA and Act7ACAC pre-mRNA showed comparable results (see fig. 30p. Even if the spliceosome could not undergo the second catalytic step due to the ACAC mutation in the 3'SS, a certain amount of Cwc25 dissociated from the spliceosome after the activity of Prp16. Further incubation with Slu7/Prp18 then led to further release of Cwc25 from the spliceosome. These measurements showed that the stable docking 
of the 3'SS into the catalytic center is the prerequisite for Cwc25's dissociation and not a productive catalysis of the second step. This model is in agreement with the results of previous studies in which the second catalytic step was inhibited by mutations in the 3'SS 61 [6]. These mutations were nevertheless bound by Slu7 and potentially at the catalytic center of the spliceosome.

In a model of the processes, the binding of Slu7/Prp18 to Actwt $\mathrm{C}^{*}$ spliceosomes leads indirectly to the release of Cwc25. The primary task of Slu7/Prp18 is efficiently docking of the 3'SS into the catalytic center for the second step. A possible further function of Slu7/Prp18 is the triggering of a conformational change in the catalytic center that stabilizes the step 2 conformation and destabilizes the binding of Cwc 25 in the catalytic center. The FCCS measurements on the C complexes assembled on Act7 pre-mRNA showed that this possible function of Slu7/Prp8 is not a critical one.

To conclude, the results indicate that the second catalytic step is not the trigger for the release of Cwc25 from the spliceosome. The FCCS measurements suggest that the activity of Prp16 is a prerequisite for the release of Cwc25. For complexes assembled on Actwt pre-mRNA, the action of Slu7/Prp18 leads to the dissociation of Cwc25. The distance between BS and 3'SS strongly influences the requirements for Cwc25s release. If the distance is short, the 3'SS can assume a position close to the active site. Upon ATP hydrolysis by Prp16, the spliceosome is rearranged with the 3'SS still in the catalytic center. In this case, the 3'SS is close enough to the catalytic center and the step 2 active site without further stabilization by Slu7/Prp18. The activity of Prp16 alone and the docking of the 3'SS into the active site is sufficient for the dissociation of Cwc25. Further addition of Slu7/Prp18 then stabilizes the step 2 conformation, facilitates the docking event, and thereby the further release of Cwc25 from the spliceosome.

A previous study of Tseng et al. showed a different behavior. Using immunoprecipitation experiments, they monitored that Cwc25 is displaced after the action of Prp16 alone. They performed experiments in the presence and in the absence of ATP and showed that the function of Prp16 is ATP dependent 69. The experiments were performed at high salt concentrations of $200 \mathrm{mM} \mathrm{KCl}$. It was shown by several FCCS experiments in this study that the binding affinity of one protein varies for different salt concentrations. Higher salt concentrations correspond to harsher conditions for the binding of a protein. At $200 \mathrm{mM} \mathrm{KCl}$, Cwc25 is more readily displaced from the spliceosome. Our experiments were performed at lower and more physiological salt concentrations of $75 \mathrm{mM} \mathrm{KCl}$, which is a more gentle condition 
for the spliceosome. Another problem in the study of Tseng et al. is a possible contamination with Slu7/Prp18 due to their experimental conditions. The presence of this heterodimer can severely influence and falsify their results.

\subsubsection{Binding behavior of Slu7/Prp18 and Prp16}

Currently, it is poorly understood at which moment the step 2 factors Slu7/Prp18 and Prp16 are recruited to the spliceosome. Therefore, FCCS measurements with Slu7-eGFP and Prp16-eGFP on the $\mathrm{B}^{\text {act }}, \mathrm{B}^{*}, \mathrm{C}$, and $\mathrm{C}^{*}$ complex were performed. Experiments at low $(75 \mathrm{mM} \mathrm{KCl})$ and at high salt concentrations $(150 \mathrm{mM} \mathrm{KCl})$ were conducted to gain information about whether the binding is weak - indicated by reduced binding at $150 \mathrm{mM}$ - or strong - indicated by comparable binding levels at both concentrations.

At $75 \mathrm{mM} \mathrm{KCl,} \mathrm{a} \mathrm{high} \mathrm{cross-correlation} \mathrm{amplitude} \mathrm{for} \mathrm{Slu7-eGFP} \mathrm{from} \mathrm{the} \mathrm{B*} \mathrm{to}$ the post catalytic (PC) spliceosome could be observed but a low cross-correlation amplitude for $\mathrm{B}^{\text {act }}$ complexes (see fig. 32 . When the salt concentration was increased to $150 \mathrm{mM} \mathrm{KCl}$, Slu7-eGFP remained only stable at $\mathrm{C}^{*} / \mathrm{PC}$ but not at the previous steps like $\mathrm{B}^{*}$ and $\mathrm{C}$, shown by low cross-correlation amplitudes which were comparable to the background. The binding strength of Slu7 to these complexes is weak, because the binding which is present at low salt concentration is disrupted at harsher conditions. In contrast, the observed cross-correlation amplitude for the $\mathrm{C}^{*} / \mathrm{PC}$ complex was high at both conditions demonstrating that the binding of Slu7 to this latter complex appears to be stronger.

As a control of the functionality of the assay, it was investigated if Slu7 can bind without Prp18 to the spliceosome. For complexes supplemented with Slu7 but without Prp18 at $75 \mathrm{mM} \mathrm{KCl,} \mathrm{a} \mathrm{low} \mathrm{cross-correlation} \mathrm{amplitude} \mathrm{was} \mathrm{observed.} \mathrm{This}$ result indicated that Slu7 can only bind to the spliceosome in the presence of Prp18. This is in agreement with earlier findings which showed that both proteins are only functionally active as a heterodimer 86 [56 55 .

As a further control experiment, a denaturing PAGE was performed to see if Slu7/Prp18 catalyzes the second catalytic step at low and high salt concentrations. The electrophoresis experiments showed that C complexes, in which Slu7/Prp18 has a low-affinity binding site, were able to carry out the second catalytic step after the addition of Prp16 and ATP (see fig. 33). This indicates that Slu7/Prp18's low-affinity binding site on the $\mathrm{C}$ complex is its functionally relevant site. Comparable FCCS experiments were performed with Prp16-eGFP. At $75 \mathrm{mM} \mathrm{KCl}$, 
Prp16-eGFP had similar binding efficiencies from $\mathrm{B}^{*}$ to $\mathrm{C}^{*} / \mathrm{PC}$ complexes (see fig. 34). For the binding of Prp16-eGFP to the complexes at a salt concentration of $150 \mathrm{mM} \mathrm{KCl}$, a different behavior was monitored. Prp16-eGFP was bound to the $\mathrm{B}^{*}$ complex but the binding to the $\mathrm{C}^{*}$ complex was more efficient. In comparison to the binding behavior of Slu7, the binding of Prp16 to the $\mathrm{B}^{*}$ complex was stronger, i.e. more salt-resistant because the cross-correlation amplitude for Prp16 to this complex was still higher than for Slu7 even at high salt concentrations. The data also indicated that Prp16 was still bound after the activation for the second step catalysis and was destabilized from the spliceosome after the second catalytic step. The measurements showed further that Prp16 is most stably bound to the $\mathrm{C}^{*}$ complex in which it is active. Several studies assumed a proofreading function for Prp16 because it can act during or before the first catalytic step if the splicing reaction is influenced by mutations in the BS or the U6 snRNA [87 97 98 69. Using FCCS, it was observed that Prp16 is bound to the early $\mathrm{B}^{*}$ complex but is not required for the first catalytic step. This corresponds to Prp16's proofreading function.

As the FCCS measurements showed, binding sites are generated for the step 1 factor Cwc25 and the second step factors Slu7/Prp18 and Prp16 in the B* complex. The measurements monitored further that the binding of Prp16 and Slu7/Prp18 to the $\mathrm{B}^{*}$ complex are sensitive against increasing salt concentrations, but several observations suggest that the binding is specific. The question arose whether Prp16 and Slu7 have partially overlapping or distinct binding sites on the $\mathrm{B}^{*}$ complex. Competition experiments performed with one labeled protein, e.g. Prp16-eGFP after addition of the same but unlabeled protein, i.e. Prp16, and after addition of the other unlabeled protein, i.e. Slu7/Prp18, showed that Prp16's entry site on the B* complex is distinct from Slu7/Prp18's entry site and they do not overlap (see fig. 35 and 36). Because Slu7/Prp18 bound with the same efficiency to the B* and C complex (which contains Cwc25), it was assumed that binding sites of Cwc25 and Slu7/Prp18 on the C complex do not overlap. Otherwise, the cross-correlation amplitude for the C complex incubated with Slu7/Prp18 would have to decrease if Cwc25 was bound to the complex. This was not observed and Cwc25 cannot be dissociated from the spliceosome by competing for its binding site with Slu7/Prp18. The advantage of binding sites for Prp16 and Slu7/Prp18 at the early stage of the $\mathrm{B}^{*}$ complex could be that the step 2 factors are already present in the spliceosome before their catalytic function is needed during the second step. 


\subsubsection{Model for the binding and activity of the step 1 and step 2 splicing factors}

Based on the findings shown in this work on the dynamic behavior of spliceosomal proteins to and from the spliceosome, the following model of substantial changes from step 1 to step 2 catalysis can be made. The catalytic steps are shown schematically in figure 37 .

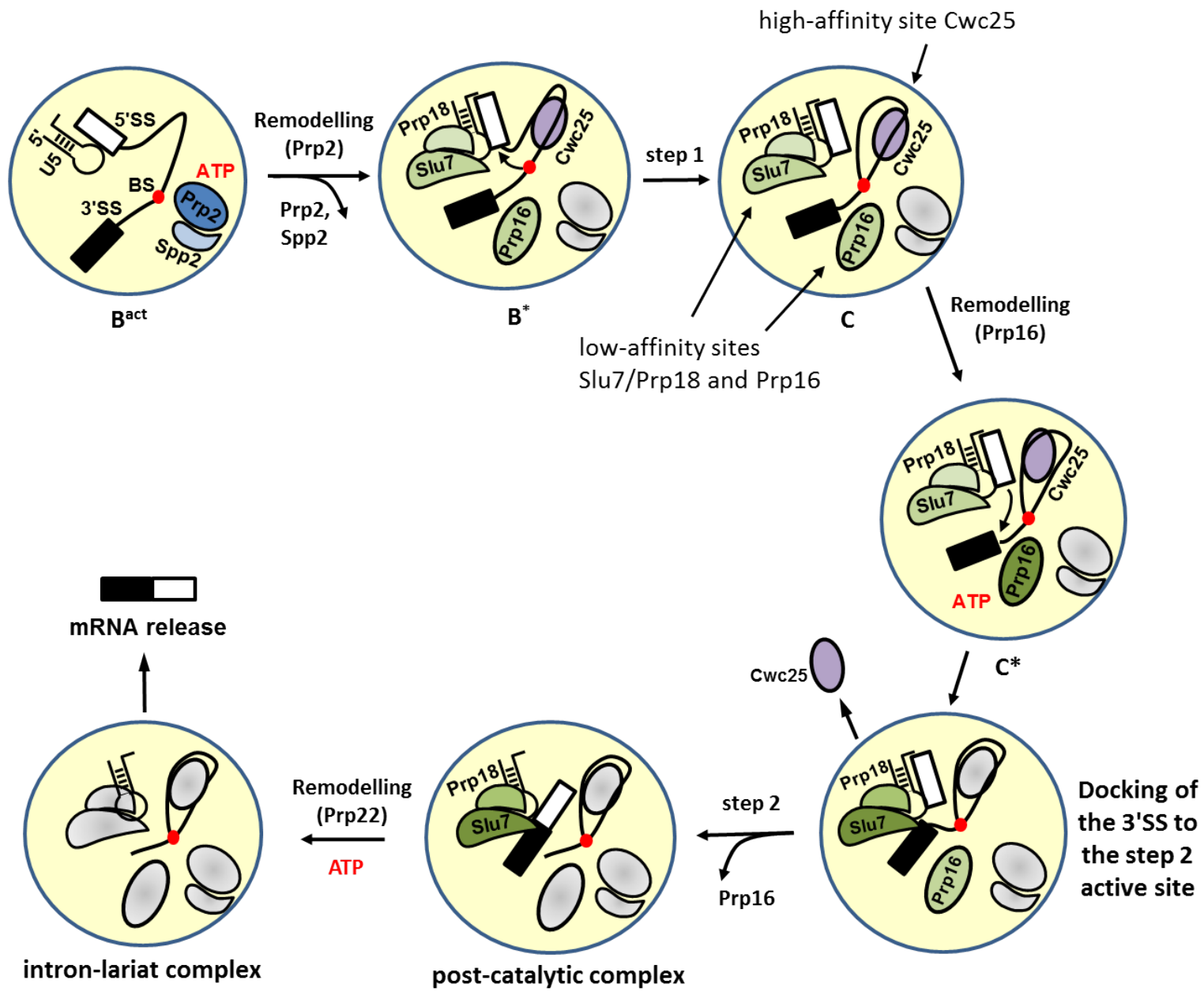

Figure 37: Schematic representation of the binding and activity of step 1 and step 2 factors during the catalytic steps of the spliceosome. (Figure adapted from Ohrt et al. ${ }^{84]}$ )

In the first step, a high-affinity binding site is generated for Cwc25 and low-affinity binding sites are generated for Slu7/Prp18 and Prp16 by the Prp2-mediated catalytic activation of the $\mathrm{B}^{\text {act }}$ complex. After binding of the step 1 factor Cwc25, the first catalytic step proceeds. This leads to the formation of the $\mathrm{C}$ complex in which $\mathrm{Cwc} 25$ is stably bound. During this processes, a conformational change is likely to occur. The low-affinity binding site of Prp16 is transformed into a high-affinity binding site 
which resists high salt concentrations of $150 \mathrm{mM} \mathrm{KCl}$. Prp16 hydrolyzes ATP and concomitantly the catalytic center is transformed from the step 1 into the step 2 conformation. The binding affinity of Cwc25 is reduced due to the rearrangements but is still bound. Due to Prp16's ATPase activity, the low-affinity binding site for Slu7/Prp18, which is also Slu7/Prp18's entry site, is transformed into a high-affinity one shown by the increased salt resistance of Slu7/Prp18's binding, and the efficient docking of the 3'SS into the step 2 active site.

Most likely by the docking of the 3'SS into the active site, a conformational change at the catalytic center is induced. This leads to the dissociation of Cwc25 and the second catalytic step can occur. Prp16 is bound to the $\mathrm{C}^{*}$ complex after hydrolyzing ATP and dissociates when Slu7/Prp18 is stably bound to the complex.

Our results suggest that the step 2 factors and Cwc25 are bound simultaneously to the catalytically activated spliceosome. The binding site of each factor is converted or "toggled" from a low-affinity, to a high-affinity, and back to a low-affinity site. 


\section{Conclusion and outlook}

Precise binding studies in highly complex macromolecular systems are challenging to perform. In this thesis, FCCS was used to study the recruitment and dissociation of certain proteins to the spliceosome.

An FCCS setup which allows for observing binding reactions, measuring low binding constants, and distinguishing between binding sites of proteins in a complex was implemented. A statement whether a protein has a low- or high-affinity for a binding site could be made. These applications make it possible to gain new insight in the rearrangements during the catalytic steps of the spliceosome.

With FCCS, it could be shown that Cwc25 is bound with a high-affinity to the spliceosome after the Prp2-mediated rearrangements of the $\mathrm{B}^{\text {act }}$ complex into the catalytically activated $\mathrm{B}^{*}$ complex. By the activity of Prp16, local rearrangements of the catalytic center from the step 1 to the step 2 conformation are induced. The primary task of Slu7/Prp18 is then the docking of the 3'SS into the step 2 catalytic center, which leads to the dissociation of Cwc25. It was shown that Slu7/Prp18 and Prp16 bind early to distinct binding sites in the step 1-activated $\mathrm{B}^{*}$ complex. One important finding was that the low-affinity binding sites of Prp16 and Slu7 were converted into high-affinity binding sites during spliceosomal maturation.

The results of the FCCS measurements provide information about the factor requirement for the second catalytic step and the dynamics of step 1 and 2 factors during the catalytic steps.

For future work, it would be interesting to investigate the recruitment and dissociation of other helicases, e.g. Prp22 and Prp43, to answer the questions: When are these helicases recruited to the spliceosome? Which proteins or helicases induce the recruitment or dissociation of Prp22 or Prp43? Is there an overlap between their binding sites? All these questions can be again answered by FCCS and the established experiments in this work. It would be interesting to determine the binding constants for other proteins to gain comprehensive information about the regulation of binding and dissociation of proteins in the spliceosome.

Another interesting aspect is the positioning of the step 1 and step 2 factors to each other during the catalytic steps. With FCCS, information about the recruitment and dissociation order of the splicing factors and their binding affinities can be obtained but not how the positions and orientations of the proteins within the spliceosome change during the catalytic steps. Is the distance between Prp16 and Slu7/Prp18 altering during the rearrangements? What is the distance between Cwc25 and Prp16? 
One possibility to determine distances between certain proteins is given by Förster Resonance Energy Transfer (FRET). This is a process which describes the energy transfer between two fluorophores. Through dipole-dipole coupling, a donor fluorphore in its electronically excited state may transfer energy to an acceptor fluorphore. The efficiency of the energy transfer is inversely proportional to the sixth power of the distance between donor and acceptor. This makes FRET useful for determining small distances between a molecule carrying the donor fluorophore, e.g. Prp16, and a molecule carrying the acceptor fluorophore, e.g. Slu7/Prp18.

It would be interesting to determine changes in the distances of e.g. Prp2 and Spp2 from one stage of the splice cycle to another one. Other possible protein pairs are Cwc22 and Cwc25, Snu114 and Cwc24, or Slu7/Prp18 and Prp16.

During the implementation of FRET for studies on the spliceosome, several aspects have to be considered. First of all, suitable donor and acceptor fluorophores have to be chosen. The so-called Förster radius, i.e. the distance at which the energy transfer efficiency is $50 \%$, has to match the distances under investigation. With standard FRET pairs, distances between 1-10 nm can be observed, which is the distance range that would be expect in the catalytic center of the spliceosome.

Second, the orientation between both fluorophores has to be considered. This is taken into account by the orientation factor $\kappa^{2}$, which depends of the relative orientation of the donor's emission dipole moment and the acceptor's absorption dipole moment. For freely rotating fluorophores, $\kappa^{2}$ adopts a value of $2 / 3$. For the spliceosome, however, which is a complex system, the assumption of $\kappa^{2}=2 / 3$ does not hold. For such complex systems values for $\kappa^{2}$ between 0 and 4 have to be assumed depending on the orientation of the fluorophores in the system. Until now it is not possible to determine the orientation factor with experimental methods.

The distance between both fluorophores depends on the Förster radius which in turn depends on the orientation factor. It is important that the fluorophores are attached via a flexible linker to the molecules of interest, since for freely rotating dyes, a value of $2 / 3$ can be assumed for the orientation factor and absolute distances can be observed. Otherwise, only the relative movement of two molecules to each other can be determined. When choosing fluorescent dyes with the appropiate Förster distance and orientations for the proteins of the spliceosome, the used FCCS setup offers the possibility to perform FRET measurements on spliceosomal complexes. 


\section{Appendix}

\section{Bibliography}

[1] D. Magde, E. Elson, W. W. Webb, "Thermodynamic fluctuations in a reacting system - measurement by fluorescence correlation spectroscopy" Phys. Rev. Lett. (1972) 29, 705-708.

[2] D. Magde, E. Elson, W. W. Webb, "Fluorescence Corelation Spectroscopy II. An Experimental Realization" Biopolymers (1974) 13, 29-61.

[3] E. L. Elson, D. Magde, "Fluorescence Corelation Spectroscopy I. Conceptual Basis and Theory" Bioploymers (1974) 13, 1-27.

[4] J. Widengren, R. Rigler, U Mets, "Triplet-state Monitoring by Fluorescence Correlation Spectroscopy" J Fluoresc (1994) 4, 255-258.

[5] P. Schwille, S. Kummer, A. A. Heikal, W. E. Moerner, W. W. Webb, "Fluorescence correlation spectroscopy reveals fast optical excitation-driven intramolecular dynamics of yellow fluorescent proteins" PNAS (2000) 97(1), 151-156.

[6] J. Widengren, U Mets, R. Rigler, "Photodynamic properties of green fluorescent proteins investigated by fluorescence correlation spectroscopy" Chem Phys (1999) 250, 171-186.

[7] L. Edman, Z. Földes-Papp, S. Wennmalm, R. Rigler, "The fluctuating enzyme: a single molecule approach" Chem Phys (1999) 247, 11-22.

[8] S. Wennmalm, L. Edman, R. Rigler, "Conformational Fluctuations In Single DNA-Molecules" PNAS (1997) 94, 10641-10646.

[9] P. Schwille, F. Oehlenschläger, N. G. Walter, "Quantitative Hybridization Kinetics of DNA Probes to RNA in Solution Followed by Diffusional Fluorescence Correlation Analysis" Biochemistry (1996) 35(31), 10182-10193.

[10] U. Kettling, A. Koltermann, P. Schwille, M. Eigen, "Real-time enzyme kinetics monitored by dual-color fluorescence cross-correlation spectroscopy" PNAS (1998) 95(4), 1416-1420. 
[11] M. Kinjo, G. Nishimura, T. Koyama, U. Mets, R. Rigler, "Single-molecule analysis of restriction DNA fragments using fluorescence correlation spectroscopy" Anal Biochem (1998) 260, 166-172.

[12] M. Rarbach, U. Kettling, A. Koltermann, M. Eigen, "Dual-Color Fluorescence Cross-Correlation Spectroscopy for Monitoring the Kinetics of EnzymeCatalyzed Reactions" Methods (2001) 24(2), 104-116.

[13] J. Lakowicz, Principles of Fluorescence Spectroscopy, Third Edition, Springer Science + Business Media New York (2006).

[14] T. Weidemann, P. Schwille, "Chapter Three - Dual-Color Fluorescence CrossCorrelation Spectroscopy with Continuous Laser Excitation in a Confocal Setup" Methods in Enzymology (2013) Volume 518, 43-70.

[15] Z. Földes-Papp, "How the molecule number is correctly quantified in twocolor fluorescence cross-correlation spectroscopy: corrections for cross-talk and quenching in experiments." Curr Pharm Biotechnol. (2005) 6(6), 437-44.

[16] P. J. Walla, Modern Biophysical Chemistry - Detection and Analysis of Biomolecules, WILEY-VCH Weinheim (2009).

[17] Földes-Papp Z., Rigler R., "Quantitative two-color fluorescence crosscorrelation spectroscopy in the analysis of polymerase chain reaction." Biol Chem (2001) 382(3), 473-8.

[18] M. Eigen, R. Rigler, "Sorting single molecules: Application to diagnostics and evolutionary biotechnology" PNAS (1994) 91, 5740-5747.

[19] P. Schwille, F. J. Meyer-Almes, R. Rigler, "Dual-color fluorescence crosscorrelation spectroscopy for multicomponent diffusional analysis in solution" Biophys J (1997) 72, 1878-1886.

[20] R. Rigler, Z. Foeldes-Papp, F. J. Meyer-Almes, C. Sammet, M. Voelcker, A. Schnetz, "Fluorescence cross correlation: A new concept for polymerase chain reaction" J Biotechnol (1998) 63, 97-109.

[21] J. Bieschke, A. Giese, W. Schulz-Schäffer, I. Zerr, S. Poser, M. Eigen, H. Kretzschmar, "Ultrasensitive detection of pathological prion protein aggregates by dual-color scanning for intensely fluorescent targets" PNAS (2000) 97, 54685473. 
[22] L. T. Varghese, R. K. Sinha, J. Irudayaraj, "Study of binding and denaturation dynamics of $\operatorname{IgG}$ and anti-IgG using dual color fluorescence correlation spectroscopy" Analytica Chimica Acta (2008) 625(1), 103-109.

[23] T. Weidemann, M. Wachsmuth, M. Tewes, K. Rippe, J. Langowski, "Analysis of Ligand Binding by Two-Colour Fluorescence Cross-Correlation Spectroscopy" Single Molecules (2002) 3, 49-61.

[24] K. Bacia, P. Schwille, "Practical guidelines for dual-color fluorescence crosscorrelation spectroscopy" Nature Protocols (2007) 2(11), 2842-2856.

[25] E. Thews, M. Gerken, R. Eckert, J. Zaepfel, C. Tietz, J. Wrachtrup, "Cross Talk Free Fluorescence Cross-Correlation Spectroscopy in Live Cells" Biophys $J(2005)$ 89, 2069-2076.

[26] S. Maiti, U. Haupts, W. W. Webb, "Fluorescence correlation spectroscopy: Diagnostics for sparse molecules" PNAS (1997) 94, 11753-11757.

[27] P. Schwille, U. Haupts, S. Maiti, W. W. Webb, "Molecular dynamics in living cells observed by fluorescence correlation spectroscopy with one- and twophoton excitation" Biophysical Journal (1999) 77(4), 2251-2265.

[28] Y. H. Foo, N. Naredi-Rainer, D. C. Lamb, S. Ahmed, T. Wohland, "Factors Affecting the Quantification of Biomolecular Interactions by Fluorescence CrossCorrelation Spectroscopy" Biophysical Journal (2012) 102(5), 1174-1183.

[29] L. C. Hwang, T. Wohland, "Dual-Color Fluorescence Cross-Correlation Spectroscopy Using Single Laser Wavelength Excitation" ChemPhysChem (2004) $5(4), 549-551$.

[30] L. C. Hwang, T. Wohland, "Single wavelength excitation fluorescence crosscorrelation spectroscopy with spectrally similar fluorophores: Resolution for binding studies" JCP (2005) 122, 114708.

[31] K. Heinze, A. Koltermann, P. Schwille, "Simultaneous two-photon excitation of distinct labels for dual-color fluorescence crosscorrelation analysis" PNAS (2000) 97, 10377-10382.

[32] S. A. Kim, K. G. Heinze, K. Bacia, M. N. Waxham, P. Schwille, "Two-photon cross-correlation analysis of intracellular reactions with variable stoichiometry" Biophysical Journal (2005) 88(6), 4319-4336. 
[33] D. Elliott, M. Ladomery, Molecular Biology of RNA, Oxford Univ. Press Oxford (2011).

[34] G. Karp, Cell and molecular biology: concepts and experiments - 3rd edition, John Wiley \& Sons, Inc. New York (2002).

[35] P. J. Lopez, B. Seraphin, "Genomic-scale quantitative analysis of yeast premRNA splicing: implications for splice-site recognition" $R N A$ (1999) 5(9), 1135-1137.

[36] P. J. Lopez, B. Seraphin, "YIDB: the Yeast Intron DataBase" Nucleic Acids Research (2000) 28(1), 85-86.

[37] P. Fabrizio, J. Dannenberg, P. Dube, B. Kastner, H. Stark, H. Urlaub, R. Lührmann, "The Evolutionarily Conserved Core Design of the Catalytic Activation Step of the Yeast Spliceosome" Molecular Cell (2009) 36(4), 593608.

[38] B. Alberts, A. Johnson, J. Lewis, M. Raff, K. Roberts, P. Walter, Molecular biology of the cell - 5th edition, Garland Science, Taylor \& Francis Group New York (2007).

[39] G. M. Cooper, R.E. Hausman, The Cell: A Molecular Approach - 5th edition, ASM Press Washington (2009).

[40] C. L. Will, R. Lührmann, "Spliceosome Structure and Function" Cold Spring Harbor Perspectives in Biology (2011) 3(7).

[41] M. J. Moore, P. A. Sharp, "Evidence for two active sites in the spliceosome

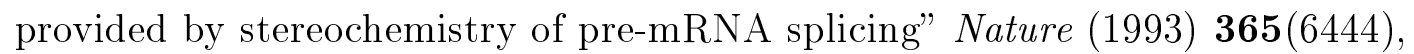
364-368.

[42] M. Sammeth, S. Foissac, R. Guigo, "A General Definition and Nomenclature for Alternative Splicing Events" PLoS Comput Biol (2008) 4(8), e1000147.

[43] O. Cordin, D. Hahn, J. D. Beggs, "Structure, function and regulation of spliceosomal RNA helicases" Current Opinion in Cell Biology (2012) 24(3), 431-438.

[44] J. P. Staley, C. Guthrie, "Mechanical Devices of the Spliceosome: Motors, Clocks, Springs, and Things" Cell (1998) 92(3), 315-326. 
[45] E. J. Sontheimer, J. A. Steitz, "The U5 and U6 small nuclear RNAs as active site components of the spliceosome" Science (1993) 262(5142), 1989-96.

[46] D. S. Horowitz, "The mechanism of the second step of pre-mRNA splicing" Wiley Interdisciplinary Reviews: RNA (2012) 3(3), 331-350.

[47] C. Chen, W. Yu, T. Tsao, L. Wang, H. Chen, J. Lin, W. Tsai, S. Cheng, "Functional and physical interactions between components of the Prp19p-associated complex" Nucleic Acids Research (2002) 30(4), 1029-1037.

[48] Z. Warkocki, P. Odenwälder, J. Schmitzova, F. Platzmann, H. Stark, H. Urlaub, R. Ficner, P. Fabrizio, R. Lührmann, "Reconstitution of both steps of Saccharomyces cerevisiae splicing with purified spliceosomal components" Nat Struct Mol Biol (2009) 16(12), 1237-1243.

[49] J. Roy, K. Kim, J. R. Maddock, J. G. Anthony, J. L. Woolford, "The final stages of spliceosome maturation require Spp2p that can interact with the DEAH box protein Prp2p and promote step 1 of splicing" $R N A$ (1995) 1(4), 375-90.

[50] S. H. Kim, R. J. Lin, "Spliceosome activation by PRP2 ATPase prior to the first transesterification reaction of pre-mRNA splicing" Molecular and Cellular Biology (1996) 16(12), 6810-9.

[51] E. J. Silverman, A. Maeda, J. Wei, P. Smith, J. D. Beggs, R. Lin, "Interaction between a G-Patch Protein and a Spliceosomal DEXD/H-Box ATPase That Is Critical for Splicing" Molecular and Cellular Biology (2004) 24(23), 1010110110.

[52] Y. Chiu, Y. Liu, T. Chiang, T. Yeh, C. Tseng, N. Wu, S. Cheng, "Cwc25 Is a Novel Splicing Factor Required after Prp2 and Yju2 To Facilitate the First Catalytic Reaction" Molecular and Cellular Biology (2009) 29(21), 5671-5678.

[53] R. M. Lardelli, J. X. Thompson, J. R. Yates, S. W. Stevens, "Release of SF3 from the intron branchpoint activates the first step of pre-mRNA splicing" $R N A$ (2010) 16(3), 516-528.

[54] M. Prior, T. Ohrt, J. Dannenberg, P. Odenwälder, O. Dybkov, N. Rasche, J. Schmitzova, I. Gregor, P. Fabrizio, J. Enderlein, R. Lührmann, "Prp2mediated protein rearrangements at the catalytic core of the spliceosome as revealed by dcFCCS" $R N A$ (2012) 18(6), 1244-1256. 
[55] B. Schwer, C. Guthrie, "A conformational rearrangement in the spliceosome is dependent on PRP16 and ATP hydrolysis" EMBO J (1992) 11(13), 5033-9.

[56] S. James, W. Turner, B. Schwer, "How Slu7 and Prp18 cooperate in the second step of yeast pre-mRNA splicing" RNA (2002) 8(8), 1068-1077.

[57] B. Schwer, "A Conformational Rearrangement in the Spliceosome Sets the Stage for Prp22-Dependent mRNA Release" Molecular Cell (2008) 30(6), 743-754.

[58] R.-T. Tsai, R.-H. Fu, F.-L. Yeh, C.-K. Tseng, Y.-C. Lin, Y. Huang, S.-C. Cheng, "Spliceosome disassembly catalyzed by Prp43 and its associated components Ntr1 and Ntr2" Genes \& Development (2005) 19(24), 2991-3003.

[59] M. C. Wahl, C. L. Will, R. Lührmann, "The Spliceosome: Design Principles of a Dynamic RNP Machine" Cell (2009) 136(4), 701-718.

[60] S. Valadkhan, Y. Jaladat, "The spliceosomal proteome: At the heart of the largest cellular ribonucleoprotein machine" PROTEOMICS (2010) 10(22), $4128-4141$.

[61] J. G. Umen, C. Guthrie, "Prp16p, Slu7p, and Prp8p interact with the 3" splice site in two distinct stages during the second catalytic step of pre-mRNA splicing" RNA (1995) 1(6), 584-597.

[62] D. S. McPheeters, B. Schwer, P. Muhlenkamp, "Interaction of the yeast DExHbox RNA helicase Prp22p with the 3' splice site during the second step of nuclear pre-mRNA splicing" Nucleic Acids Research (2000) 28(6), 1313-1321.

[63] M. Company, J. Arenas, J. Abelson, "Requirement of the RNA helicase-like protein PRP22 for release of messenger RNA from spliceosomes" Nature (1991) 349(6309), 487-493.

[64] B. Schwer, C.H. Gross, "Prp22, a DExH-box RNA helicase, plays two distinct roles in yeast pre-mRNA splicing" EMBO J (1998) 17(7), 2086-2094.

[65] A. Ansari, B. Schwer, "SLU7 and a novel activity, SSF1, act during the PRP16dependent step of yeast pre-mRNA splicing" EMBO J (1995) 14(16), 4001-9.

[66] A. Brys, B. Schwer, "Requirement for SLU7 in yeast pre-mRNA splicing is dictated by the distance between the branchpoint and the 3' splice site" RNA (1996) 2(7), 707-717. 
[67] T. A. Steitz, J. A. Steitz, "A general two-metal-ion mechanism for catalytic RNA" Proc Natl Acad Sci U S A (1993) 90(14), 6498-502.

[68] M. M. Konarska, C. C. Query, "Insights into the mechanisms of splicing: more lessons from the ribosome" Genes \&f Development (2005) 19(19), 2255-2260.

[69] C. Tseng, H. Liu, S. Cheng, "DEAH-box ATPase Prp16 has dual roles in remodeling of the spliceosome in catalytic steps" $R N A$ (2011) $\mathbf{1 7}(1), 145-154$.

[70] A. Nagy, J. R. Wu, K. M. Berland, "Characterizing observation volumes and the role of excitation saturation in one-photon fluorescence fluctuation spectroscopy" Journal of Biomedical Optics (2005) 10(4), 044015.

[71] R. Rigler, E.S. Elson, Fluorescence correlation spectroscopy: theory and application, Springer Berlin Heidelberg (2001).

[72] H. Qian, E. L. Elson, "Analysis of confocal laser-microscope optics for 3-D fluorescence correlation spectroscopy" Appl Opt (1991) 30, 1185-1195.

[73] R. Rigler, U. Mets, J. Widengren, P. Kask, "Fluorescence Correlation Spectroscopy with High Count Rate and Low-Background - Analysis of Translational Diffusion" European Biophysics Journal with Biophysics Letters (1993) 22(3), 169-175.

[74] J. Enderlein, W. P. Ambrose, "The optical collection efficiency function in single molecule detection experiments" Appl. Opt. (1997) 36, 5298-5302.

[75] B. K. Müller, E. Zaychikov, C. Brauchle, D. C. Lamb, "Pulsed interleaved excitation" Biophysical Journal (2005) 89(5), 3508-3522.

[76] T. Dertinger, V. Pacheco, I. von der Hocht, R. Hartmann, I. Gregor, J. Enderlein, "Two-Focus Fluorescence Correlation Spectroscopy: A New Tool for Accurate and Absolute Diffusion Measurements" ChemPhysChem (2007) 8, 433-443.

[77] T. Dertinger, A. Loman, B. Ewers, C.B. Müller, B. Krämer, J. Enderlein, "The optics and performance of dual-focus fluorescence correlation spectroscopy" Opt. Express (2008) 16, 14535-14368.

[78] M. Wahl, I. Gregor, M. Patting, J. Enderlein, "Fast calculation of fluorescence correlation data with asynchronous time-correlated single-photon counting" Opt. Express (2003) 11, 3583-3591. 
[79] S. L. Yean, R. J. Lin, "U4 small nuclear RNA dissociates from a yeast spliceosome and does not participate in the subsequent splicing reaction" Molecular and Cellular Biology (1991) 11(11), 5571-5577.

[80] J. Sambrook, D. W. Russell, Molecular Cloning: A Laboratory Manual, Cold Springer Harbor Laboratory Cold Spring Habor, New York (1989).

[81] A. Gottschalk, G. Neubauer, J. Banroques, M. Mann, R. Lührmann, P. Fabrizio, "Identification by mass spectrometry and functional analysis of novel proteins of the yeast [U4/U6·U5] tri-snRNP" EMBO J (1999) 18(16), 45354548.

[82] C. Bartels, H. Urlaub, R. Lührmann, P. Fabrizio, "Mutagenesis Suggests Several Roles of Snu114p in Pre-mRNA Splicing" Journal of Biological Chemistry (2003) 278(30), 28324-28334.

[83] T.-C. Yeh, H.-L. Liu, C.-S. Chung, N.-Y. Wu, Y.-C. Liu, S.-C. Cheng, "Splicing Factor Cwc22 Is Required for the Function of Prp2 and for the Spliceosome To Escape from a Futile Pathway" Molecular and Cellular Biology (2011) 31(1), $43-53$.

[84] T. Ohrt, P. Odenwälder, J. Dannenberg, M. Prior, Z. Warkocki, J. Schmitzova, R. Karaduman, I. Gregor, J. Enderlein, P. Fabrizio, R. Lührmann, "Molecular dissection of step 2 catalysis of yeast pre-mRNA splicing investigated in a purified system" RNA (2013).

[85] D. D. Boos, "Introduction to the Bootstrap World" Statistical Science (2003) 18(2), 168-174.

[86] X. Zhang, B. Schwer, "Functional and physical interaction between the yeast splicing factors Slu7 and Prp18" Nucleic Acids Res (1997) 25(11), 2146-52.

[87] S. Burgess, J. R. Couto, C. Guthrie, "A putative ATP binding protein influences the fidelity of branchpoint recognition in yeast splicing" Cell (1990) 60(5), 705717.

[88] S. M. Burgess, C. Guthrie, "A mechanism to enhance mRNA splicing fidelity: The RNA-dependent ATPase Prp16 governs usage of a discard pathway for aberrant lariat intermediates" Cell (1993) 73(7), 1377-1391. 
[89] M. Meyer, M. Plass, J. Perez-Valle, E. Eyras, J. Vilardell, "Deciphering 3'ss Selection in the Yeast Genome Reveals an RNA Thermosensor that Mediates Alternative Splicing" Molecular Cell (2011) 43(6), 1033-1039.

[90] J. Fourmann, J. Schmitzova, H. Christian, H. Urlaub, R. Ficner, K. Boon, P. Fabrizio, R. Lührmann, "Dissection of the factor requirements for spliceosome disassembly and the elucidation of its dissociation products using a purified splicing system" Genes \& Development (2013) 27(4), 413-428.

[91] D. Frank, B. Patterson, C. Guthrie, "Synthetic lethal mutations suggest interactions between U5 small nuclear RNA and four proteins required for the second step of splicing" Molecular and Cellular Biology (1992) 12(11), 5197-5205.

[92] S. Teigelkamp, A. J. Newman, J. D. Beggs, "Extensive interactions of PRP8 protein with the 5' and 3' splice sites during splicing suggest a role in stabilization of exon alignment by U5 snRNA" EMBO J (1995) 14(11), 2602-12.

[93] C. A. Collins, C. Guthrie, "Allele-specific genetic interactions between Prp8 and RNA active site residues suggest a function for Prp8 at the catalytic core of the spliceosome" Genes \& Development (1999) 13(15), 1970-1982.

[94] R. J. Grainger, J. D. Beggs, "Prp8 protein: At the heart of the spliceosome" $R N A(2005) \mathbf{1 1}(5), 533-557$.

[95] A. Aronova, D. Bacikova, L. B. Crotti, D. S. Horowitz, B. Schwer, "Functional interactions between Prp8, Prp18, Slu7, and U5 snRNA during the second step of pre-mRNA splicing" RNA (2007) 13(9), 1437-1444.

[96] L. B. Crotti, D. Bacikova, D. S. Horowitz, "The Prp18 protein stabilizes the interaction of both exons with the U5 snRNA during the second step of premRNA splicing" Genes \& Development (2007) 21(10), 1204-1216.

[97] P. Koodathingal, T. Novak, J. A. Piccirilli, J. P. Staley, "The DEAH Box ATPases Prp16 and Prp43 Cooperate to Proofread 5' Splice Site Cleavage during Pre-mRNA Splicing" Molecular Cell (2010) 39(3), 385-395.

[98] D. S. Horowitz, "The splice is right: Guarantors of fidelity in pre-mRNA splicing" $R N A(2011) \mathbf{1 7}(4), 551-554$. 


\section{List of Figures}

1 If the two fluorescently labeled molecules are not bound to each other,

\begin{tabular}{|c|}
\hline the cross-correlation amplitude is small. If the molecules are bound, \\
\hline
\end{tabular}

the cross-correlation amplitude is large. . . . . . . . . . . . . . 4

2 An overview of information flow from genes to proteins. . . . . . . . 10

3 Schematic representation of conserved sequences in introns of S. cere-

visiae and homo sapiens. . . . . . . . . . . . . . . . . 12

4 Principle of pre-mRNA splicing. . . . . . . . . . . . . . . . 13

5 Scheme of the different modes of splicing. . . . . . . . . . . . . . . 14

$6 \quad$ The assembly cycle of the spliceosome. (Figure taken from ${ }^{[40}$ ) . . . . 16

7 Compositional dynamics of yeast spliceosomes. . . . . . . . . . . . 17

8 The two-state model for the catalytic steps. . . . . . . . . . . . . 20

9 Spliceosomal complexes to which the binding of several proteins was

observed with FCCS. . . . . . . . . . . . . . . . . . . 21

10 Confocal microscope setup for Dual-Color Fluorescence Cross-Correlation

Spectroscopy. . . . . . . . . . . . . . . . . . . 24

11 Detection principle of Dual-Color Fluorescence Cross-Correlation Spec-

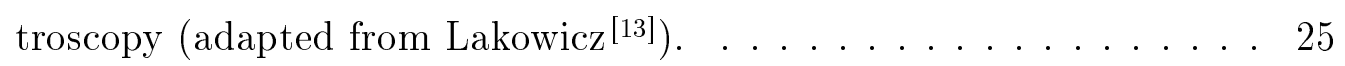

12 Principle of FCCS. . . . . . . . . . . . . . . . . . 26

13 Example of an auto-correlation curve with the diffusion time $\tau_{D}$. . . 28

14 Emission and transmission spectra. . . . . . . . . . . . . . . 34

15 Schematic picture of the 2fFCS setup. . . . . . . . . . . . . 35

16 Schematic of a Nomarski prism. . . . . . . . . . . . . 36

17 Exemplary representation of correlation curves in an FCCS experiment. 38

18 The used optical setup for FCCS and 2fFCS experiments. . . . . . . . 39

19 Schematic representation of the experimental design and data. . . . . 50

20 Binding behavior of Cwc22-eGFP to the $\mathrm{B}^{\text {act }}$ complex at time point

zero and after 180 min. For each data point, the mean and the stan-

dard deviation of two independent measurements were calculated. . . 51

21 Normalized data points and fits of the cross-correlation curves for Cwc22's binding to the $\mathrm{B}^{\text {act }}$ complex at time point zero and after

$180 \mathrm{~min}$. Exemplary curves of one measurement at time point zero and of one measurement after $3 \mathrm{~h}$ are shown. . . . . . . . . . . . 51

22 Binding behavior of Snu114-eGFP. . . . . . . . . . . . . 52

23 Binding behavior of Snu114-eGFP at different salt concentrations. . . 53 
24 Binding of Cwc25-Alexa488 to Actwt pre-mRNA. . . . . . . . . . . 56

25 Titration of $\mathrm{B}^{\text {act } \Delta \operatorname{Prp} 2}$ complexes and B* complexes to Cwc25-Alexa488. 57

26 Binding of Cwc25-Alexa488 to ActACAC 3'SS mutated pre-mRNA. . 59

27 Splicing assay of spliceosomes assembled on ActACAC pre-mRNA and Actwt pre-mRNA. . . . . . . . . . . . . . . 6 60

28 Binding of Cwc25-Alexa488 to Act7wt pre-mRNA. . . . . . . . . . . 61

29 Factor requirements for the second catalytic step of complexes assembled on Act7 pre-mRNA. . . . . . . . . . . . . . . . . . . 62

30 Binding of Cwc25-Alexa488 to Act7ACAC 3'SS mutated pre-mRNA. 63

31 Functional assay for testing the functionality of Slu7-eGFP and Prp16-

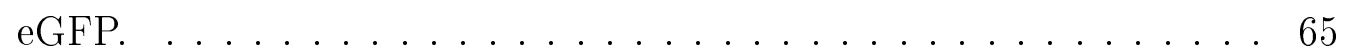

32 Binding behavior of Slu7-eGFP to the spliceosome. . . . . . . . . . 67

33 Denaturing PAGE with $\mathrm{C}$ complexes. . . . . . . . . . . . . . . 68

34 Binding behavior of Prp16-eGFP to the spliceosome. . . . . . . . . 70

35 Competition of Prp16-eGFP's binding site with the one for Slu7/Prp18. 71

36 Competition of Slu7-eGFP's binding site with the one for Prp16. . . . 72

37 Schematic representation of the binding and activity of step 1 and step 2 factors during the catalytic steps of the spliceosome. (Figure adapted from Ohrt et al. $\left.\left.{ }^{84}\right)\right] \ldots$. . . . . . . . . . . 82 


\section{Acronyms}

2fFCS Dual-Focus Fluorescence Correlation Spectroscopy

3'SS 3' Splice Site

5'SS 5' Splice Site

5'-GMPS Guanosine-5'-O-mono phosphorothioate

A Adenosine

ACF Auto-Correlation Function

ADP Adenosine-5'-diphosphate

ATP Adenosine-5'-triphosphate

bp base pairs

BS Branch Site

C Cytosine

CCF Cross-Correlation Function

CEF Collection Efficiency Function

DMF Dimethylformamide

DTT 1,4-dithiothreitol

EDTA ethylenediamine-N, N, N', N'-tetraacetic acid

eGFP enhanced green fluorescent protein

FCCS Dual-Color Fluorescence Cross-Correlation Spectroscopy

FCS Fluorescence Correlation Spectroscopy

FP $\quad$ Fluorescence Polarization

G Guanosine

GSH Gluthathione

MDF Molecular Detection Function 
mRNA messenger RNA

MS Mass Spectrometry

NP-40 Nonidet P-40

NTC Nineteen Complex

OD Optical density

PAGE Polyacrylamide gel electrophoresis

PEG Polyethylene glycol

PMSF Phenylmethylsulfonylfluorid

pre-mRNA precursor-messenger RNA

S Svedberg

snRNA small nuclear Ribonucleic Acid

snRNP small nuclear Ribonucleoprotein

T Thymidine

TCSPC Time-Correlated Single-Photon Counting

TEMED N, N, N', N'-tetramethylethylenediamine

U Uridine 


\section{Acknowledgments}

Ich möchte allen danken, die mich während meiner Doktorarbeit unterstützt und mir immer geholfen haben:

- Jörg Enderlein danke ich für die Betreuung meiner Doktorarbeit und die Möglichkeit, während dieser Zeit an vielen verschiedenen und interessanten Projekten zu arbeiten. Ich danke dir für diese abwechslungsreichen Projekte, durch die ich immer wieder etwas Neues lernen konnte. Auch für deine stets offene Tür und alle Hilfen bei Problemen rund um meine Arbeit danke ich dir sehr.

- Meinem Thesis Committee bestehend aus Reinhard Lührmann und Kai Tittmann danke ich für die Betreuung meiner Doktorarbeit und viele hilfreiche Diskussionen. Reinhard danke ich zudem für alle hilfreichen Besprechungen rund um das Spliceosom.

- Ingo Gregor danke ich für seine stets offene Tür und dafür, dass du jederzeit alle meine Fragen zu FCS, FCCS und FRET geduldig beantwortet hast. Ich danke dir dafür, dass du mir bei allen Matlab- und MicroTimeproblemen geholfen hast und ich sehr viel von dir lernen konnte.

- Ein ganz besonders großer Dank geht an Kerstin! Ich danke dir für deine Unterstützung und Freundschaft während dieser gemeinsamen Zeit. Ich freue mich sehr, dass du damals aus Aachen nach Göttingen gezogen bist und ich mit dir eine "Konferenz-und-Büro-Zimmer-Nachbarin" fürs Leben gefunden habe und dafür, dass ich dich manchmal nur anzuschauen brauche und dann weiß, dass du genau das Gleiche denkst wie ich. Du hast mir in jeder Situation beigestanden, hattest immer ein offenes Ohr für mich und hast mich immer super beruhigt und motiviert, wenn ich dachte, ich schaffe etwas nicht.

- Phillip danke ich für all seine vielen Ablenkungen durch Witze, Sprüche und Streiche, die immer zur richtigen Zeit kamen und durch die wir sehr viel Spaß während dieser gemeinsamen Zeit hatten. Aber auch für deine Unterstützung in nicht so guten Zeiten, die gemeinsamen DPG Tagungen und Unternehmungen außerhalb des Labors danke ich dir sehr. Und ich danke dir außerdem dafür, dass du uns immer mit Keksen und süßen Hundebildern versorgt hast. 
- Anja und Melanie danke ich für lustige Mittagessen, Kaffeerunden (einen Dank auch an die Wörgötters, die uns immer wieder mit interessanten Geprächen beim Kaffeetrinken und auch auf den Sommerschulen unterhalten haben), Gespräche und für all die Sportkurse, die wir zusammen durchgestanden haben.

- Thomas danke ich für seine Hilfe beim Thema Spleißosom, viele lustige Stunden an der MicroTime und das Zeitvertreiben zwischen den Messungen. Außerdem für deine, wie ich finde, nicht selbstverständliche Hilfe bei meiner Arbeit und all die Stunden, die du dafür investiert hast. Peter, Julia und Annika danke ich für die vielen unterhaltsamen Stunden im dunklen Labor und dafür, dass ihr mir immer wieder etwas Neues über die Biologie beigebracht habt.

- Allen weiteren Mitarbeitern des Instituts danke ich für die freundliche Atmosphäre. Ich möchte besonders Kathrin, Elke, Nicole, Sabine und Ursel dafür danken, dass sie mir alle Fragen rund um Dienstreisen, Formulare usw. beantwortet haben.

- Steffi W., Katha und Britta danke ich für viele Mädelsabende, Shoppingtouren und Kaffeerunden und dafür, dass man mit euch über alles reden kann. Christian, Patrick, Julian und Steffi R. danke ich für viele lustige Abende und eine schöne gemeinsame Zeit.

- Ganz besonders herzlich möchte ich mich bei meinen lieben Eltern Claudia und Karl Prior, meiner Schwester Mareike und meinem Bruder Henrik bedanken. Danke, dass ihr immer für mich da seid, wenn ich Hilfe brauchte. Ich danke euch außerdem dafür, dass ihr mir die Daumen bei allen Vorträgen gedrückt habt und mich stets in allen Situationen unterstützt habt. Ich freue mich sehr, dass ich jederzeit nach Hause kommen kann und dann stets eine offene Tür und ein offenes Ohr vorfinde.

- Matthias danke ich für die gemeinsame Zeit und seine grenzenlose Unterstützung, Motivation, Aufmunterung, Ablenkung und Verständnis in allen Situationen. Danke, dass du immer für mich da warst und bist! 


\section{Curriculum vitae}

Mira Prior

Date of birth: 13.April 1986

Place of birth: Steinheim, Westfalen

Nationality: German

\section{Education}

09/2010 - 10/2013 PhD in Biophysics, Georg-August University Göttingen

10/2007 - 07/2010 Diploma in Physics, Georg-August University Göttingen:

Diploma thesis in Biophysics, Georg-August University Goettingen:

"Zero-Mode Waveguide, DNA, Lipidvesikel -

Fortgeschrittende Fluoreszenzmikroskopie in der Biophysik"

Supervisor: Prof. Dr. Jörg Enderlein

10/2005 - 09/2007 Vordiplom in Physics, Georg-August University Göttingen

10/2005 - 07/2010 Physics studies at Georg-August University Göttingen

08/1996 - 06/2005 High School - Städtisches Gymnasium Steinheim

\section{Publications}

Ohrt, T., Prior, M., Dannenberg, J., Odenwälder, P., Dybkov, O., Rasche, N., Schmitzova, J., Gregor, I., Fabrizio, P., Enderlein, J., Lührmann, R. "Prp2-mediated protein rearrangements at the catalytic core of the spliceosome as revealed by dcFCCS". (RNA 2012)

Ohrt, T., Odenwälder, P., Dannenberg, J., Prior, M., Warkocki, Z., Schmitzova, J., Karaduman, R., Gregor, I., Enderlein, J., Fabrizio, P., Lührmann, R. "Molecular dissection of step 2 catalysis of yeast pre-mRNA splicing investigated in a purified system". (RNA 2013)

\section{Conferences}

\section{Talks}

"Single-molecule fluorescence spectroscopy of the structure and dynamics of the spliceosomal complex", 18th International Workshop on Single Molecule Spectroscopy, Berlin, 2012. 
"Single-molecule fluorescence spectroscopy of the structure and dynamics of the spliceosomal complex", DPG-Frühjahrstagung der Sektion Kondensierte Materie 2012, Berlin, 2012.

"Single-molecule fluorescence spectroscopy of the structure and dynamics of the spliceosomal complex", 8th International PhD student symposium Horizons in Molecular Biology, Goettingen, 2011.

\section{Posters}

"Sinlge-molecule fluorescence spectroscopy of compositional changes of the spliceosomal complex", DPG-Frühjahrstagung der Sektion Kondensierte Materie 2013, Regensburg, 2013.

"Sinlge-molecule fluorescence spectroscopy of compositional changes of the spliceosomal complex", Symposium SFB 860 - Molecular machines in RNA processing, translation and transport, Goettingen, 2013.

"Sinlge-molecule fluorescence spectroscopy of compositional changes of the spliceosomal complex", 9th International PhD student symposium Horizons in Molecular Biology, Goettingen, 2012.

"Single-molecule fluorescence spectroscopy of the structure and dynamics of the spliceosomal complex", 56th Annual Meeting of the Biophysical Society, San Diego, CA, USA, 2012.

"Single-molecule fluorescence spectroscopy of the structure and dynamics of the spliceosomal complex", 17th International Workshop on Single Molecule Spectroscopy, Berlin, September 2011.

"Applications for fluorescence lifetime imaging: Fast genomic characterization and lifetime measurements on zero-mode waveguides", 16th International Workshop on Single Molecule Spectroscopy, Berlin, September 2010.

"An approach for fast genomic DNA characterization: Detecting sequence dependent lifetimes of the DNA-intercalacting dye YOYO", 15th International Workshop on Single Molecule Spectroscopy, Berlin, September 2009. 